Universidad Nacional de La Plata

Facultad de Ciencias Exactas

Departamento de Física

\author{
Tesis Doctoral
}

\title{
Teorías de gauge no conmutativas: \\ Chern-Simons y Born-Infeld
}

Lic. Nicolás E. Grandi

Director: Dr. Fidel A. Schaposnik 

Al Lolo... 



\section{Resumen}

En la presente tesis doctoral, estudiaremos algunos aspectos de las teorías de campos de gauge definidas en espacio no conmutativo. Discutiremos uno de los casos más sencillos de no conmutatividad, la así llamada deformación $\theta$. Una introducción somera a las teorías de campos $\theta$-deformadas, así como su relación con la Teoría de Cuerdas y con el Efecto Hall, se dará en la Parte I de la tesis.

Los temas específicos de investigación de esta tesis corresponden al estudio algunas propiedades los modelos no conmutativos de Chern-Simons en $d=2+1$ dimensiones y de Born-Infeld en $d=3+1$.

Con respecto al primer modelo, que será estudiado en la Parte II de la tesis, demostraremos que la acción de Chern-Simons se puede obtener como la anomalía de paridad al integrar fermiones en el plano no conmutativo. Estudiaremos la definición de la acción de Chern-Simons no conmutativa en una variedad con borde, su relación con el modelo quiral no conmutativo de Wess-Zumino-Witten y con la acción de Chern-Simons usual conmutativa. Luego utilizaremos esta acción para definir de manera consistente una teoría de gravedad en espacios $\theta$-deformados de $2+1$ dimensiones.

En cuanto al modelo de Born-Infeld no conmutativo, del cual nos ocuparemos en la Parte III, estudiaremos la posibilidad de definir una acción de Born-Infeld no conmutativa, utilizando un orden simétrico en la expansión en potencias del lagrangiano, así como también su posible supersimetrización. 



\section{Agradecimientos}

A Geles, por todo y antes que a nadie. A mis padres por bancarme la carrera y a mis amigos por acompañarme en la vida.

A todos los que contribuyeron a disminuir un poco mi ignorancia. A Fidel por su apoyo, su paciencia y su profesionalismo. A Enrique, Daniel y Carlos por su buena disposición hacia mis preguntas y porque siempre tienen respuestas. A mis coautores, todos.

A Guille, claro, por aceptar discutir lo mismo cien veces hasta convencernos de haberlo entendido. Y a Leo, por volverlo a discutir.

A la enorme cantidad de gente de quien saqué provecho discutiendo algo de física: Pablo, Gastón, Mauricio, Adrián, Hugo, Martín, Diego, Gerardo, Gustavo, etc.

Agradezco al Profesor Máximo Bañados, de la Universidad Católica de Chile, por haber aceptado ser jurado de esta tesis, y extiendo este agradecimiento a los profesores Ricardo Gamboa Saraví y Huner Fanchiotti de la Universidad Nacional de La Plata. 



\section{Índice general}

1. Introducción

I Una introducción a las teorías de campos no conmutativas 19

2. Introducción a las teorías no conmutativas 23

2.1. ¿Qué es la geometría no conmutativa? . . . . . . . . . . . . . . . 23

2.2. Deformación $\theta$ y el producto ${ }^{*}$ de Moyal . . . . . . . . . . . . . . . . . 25

2.3. Teorías de campos con producto estrella . . . . . . . . . . . . . 27

3. La motivación de las teorías no conmutativas en teoría de cuerdas y en materia condensada $\quad 31$

3.1. Cuerdas abiertas en un campo de Neveu-Schwarz constante . . . . . . . . . . 31

3.2. El efecto Hall . . . . . . . . . . . . . . . . . . . . . . . . . . . . . 39

4. Las teorías de gauge no conmutativas y el mapeo de Seiberg-Witten 43

4.1. Campos de materia . . . . . . . . . . . . . . . . . . . . . . . 43

4.2. Conexión, curvatura y derivadas covariantes . . . . . . . . . . . . . 44

4.3. Acción invariante de gauge . . . . . . . . . . . . . . . . . . . 46

4.4. El mapeo de Seiberg y Witten . . . . . . . . . . . . . . . . . . . . . . 48

II Teoría de Chern-Simons en espacio no conmutativo $\quad 51$

5. Introducción a la teoría de Chern-Simons $\quad \mathbf{5 5}$

5.1. La acción de Chern-Simons en espacio conmutativo . . . . . . . . . . . . . . . 55

5.2. La acción de Chern-Simons no conmutativa . . . . . . . . . . . . . . 61

6. Término de Chern-Simons inducido por integración de fermiones $\quad 63$

6.1. Fermiones acoplados a un campo de gauge en espacio no conmutativo . . . . 63

6.2. Representaciones fundamental y antifundamental . . . . . . . . . . . . . 64

6.3. Representación Adjunta . . . . . . . . . . . . . . . . 67 


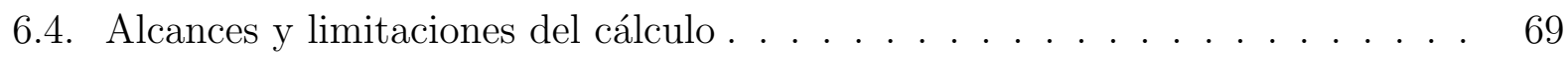

6.5. Conclusiones . . . . . . . . . . . . . . . . . . . . 71

7. La acción de Chern-Simons no conmutativa en variedades con borde 73

7.1. El efecto del borde y la derivada de la acción . . . . . . . . . . . . . . . . . 73

7.2. La conexión entre la acción de Chern-Simons y la acción quiral de WessZumino-Witten . . . . . . . . . . . . . . . . 77

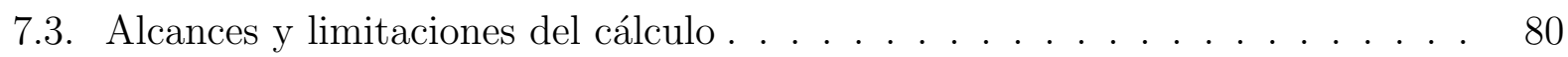

7.4. Conclusiones . . . . . . . . . . . . . . . . . . . . . . . 80

8. Comportamiento de la acción de Chern-Simons bajo el mapeo de SeibergWitten $\quad 83$

8.1. Algunas motivaciones . . . . . . . . . . . . . . . . . . . . . . 83

8.2. El mapeo de Seiberg y Witten aplicado a la acción de Chern-Simons . . . . . 84

8.3. Alcances y limitaciones del cálculo . . . . . . . . . . . . . . . . . 86

8.4. Conclusiones . . . . . . . . . . . . . . . . . . . . . . 87

9. Gravedad no conmutativa en tres dimensiones $\quad 89$

9.1. Teoría de Chern-Simons no conmutativa . . . . . . . . . . . . . . . . . 89

9.2. Gravedad tridimensional no conmutativa . . . . . . . . . . . . . . . 91

9.3. Soluciones . . . . . . . . . . . . . . . . . . . . . 95

9.4. Alcances y limitaciones de nuestra teoría . . . . . . . . . . . . . . . . . . . . . . . . . . . . . . . . . . . . . .

9.5. Conclusiones . . . . . . . . . . . . . . . . . . . . 100

III Teoría de Born-Infeld en espacio no conmutativo 103

10.Introducción a la teoría de Born-Infeld $\quad 107$

10.1. La acción de Born-Infeld conmutativa . . . . . . . . . . . . . . . . . . . . 107

10.2. La acción de Born-Infeld no conmutativa . . . . . . . . . . . . . . . 111

11.Teoría de Born-Infeld supersimétrica en espacio no conmutativo 115

11.1. Supercampos en espacio no conmutativo . . . . . . . . . . . . . . . . 115

11.2. La acción de Born-Infeld supersimétrica no conmutativa . . . . . . . . . . . 117

11.3. Supersimetría $N=2$ y ecuaciones de Bogomol'nyi . . . . . . . . . . . . . 120

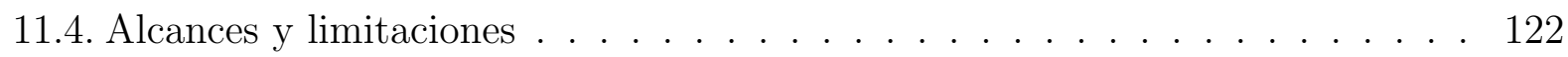

11.5. Conclusiones . . . . . . . . . . . . . . . . . . . . 122

$\begin{array}{ll}\text { 12.Conclusiones } & 125\end{array}$ 


\section{Apéndices}

13. Algunas propiedades del producto estrella

14. Notación 


\section{Capítulo 1}

\section{Introducción}

La no conmutatividad no es algo novedoso en la física: el primer ejemplo de un espacio no conmutativo que fue claramente reconocido como tal es el espacio de fases cuántico. De hecho algunas consideraciones tempranas sobre su geometría diferencial "cuantificada" fueron desarrolladas, ya en 1926, por P.A.M. Dirac [1],[2]. En estos trabajos, Dirac develó la estructura algebraica del espacio de fases cuántico, postulando su célebre regla de cuantificación de una teoría clásica, que consiste en la substitución del paréntesis de Poisson de dos observables clásicos por $i \hbar$ veces el conmutador de los operadores cuánticos asociados. De esta manera, las coordenadas del espacio de fases $p$ y $q$ se transforman en operadores no conmutantes $\hat{p}$ y $\hat{q}$ cuyo conmutador vale $i \hbar$. Esta no conmutatividad implica una relación de incerteza entre los autovalores de los operadores $\hat{p}$ y $\hat{q}$, que vacía de sentido a la noción de puntos individuales en el espacio de fases, siendo la idea más cercana que sobrevive en la teoría cuántica la de celda de Bohr de área $\hbar / 2$. En el límite $\hbar \rightarrow 0$ se recupera el espacio de fases ordinario.

Este álgebra particular de operadores fue la que inspiró la idea mas radical de substituir las coordenadas $x^{\mu}$ del espacio-tiempo por operadores no conmutantes $\hat{x}^{\mu}$. Dado que, como sucede en el caso anterior, la relación $\left[\hat{x}^{\mu}, \hat{x}^{\nu}\right] \neq 0$ implica un principio de incerteza, desdibuja la imagen del espacio-tiempo a cortas distancias. De esta manera, la idea de punto del espacio tiempo es reemplazada por la de celda de Plank de área mínima $\left\langle\left[x^{\mu}, x^{\nu}\right]\right\rangle / 2$, y el espacio tiempo carece de puntos a pequeña escala. Entonces, se puede pensar que las coordenadas $x^{\mu}$ que observamos corresponden a algún tipo de promedio sobre escalas del orden del área de Plank.

Esta idea fue inicialmente motivada en el interés por controlar las divergencias, que plagan las teorías cuánticas de campos como la electrodinámica, y que surgen en el cálculo de expresiones que contienen productos de los campos en puntos vecinos. En efecto, con el objeto de hacer finitas las integrales involucradas, es necesario truncar la integración en una 
longitud mínima $\Lambda^{-1}$, lo que introduce una escala externa en la teoría, el así llamado cutoff ultravioleta. Como fue sugerido por Snyder [3], la no conmutatividad implica necesariamente una escala por debajo de la cual no existe la noción de punto, y es posible que al introducirla en una teoría de campos proporcione un cutoff ultravioleta efectivo, es decir una distancia mínima en el espacio tiempo a la que es sensible la teoría, eliminando así los infinitos. Conviene aclarar sin embargo, que una teoría no conmutativa podría en principio tener las mismas divergencias que la teoría conmutativa o aún peores.

Luego de algunos desarrollos iniciales [4], la idea cayó en el olvido, principalmente debido a que el programa de renormalización de la teoría cuántica de campos se reveló apropiado para predecir muy precisamente valores numéricos finitos para las magnitudes observables en electrodinámica cuántica, sin recurrir a la no conmutatividad. Además, postular una relación de incerteza para las coordenadas del espacio tiempo conduce en principio a una teoría no local, con todas las complicaciones subsecuentes. Una razón suplementaria es que la no conmutatividad de las coordenadas del espacio tiempo generalmente entra en conflicto con la invarianza de Lorentz. Aún si fuera posible que la no localidad quedara confinada a escalas del orden del área de Plank, es difícil imaginar algún mecanismo que haga inobservarble la ruptura de simetría de Lorentz.

Más recientemente, una definición más formal de no conmutatividad fue desarrollada por A. Connes desde un punto de vista matemático [5]. Por algún tiempo, las aplicaciones físicas de estas ideas se basaron en la interpretación geométrica del Modelo Standard y sus múltiples campos y constantes de acoplamiento [6]-[8], donde la gravedad fue introducida de una manera unificada [9]-[14]. La idea central de estos enfoques es una generalización del mecanismo de Kaluza-Klein, donde las dimensiones ocultas se reemplazan por estructuras no conmutativas. Por ejemplo, en esta interpretación del Modelo Standard, el campo de Higgs es un campo de gauge discreto sobre la "variedad no conmutativa" $Z_{2}$, referido como un grado de libertad del tipo interno de Kaluza-Klein. Esto provee una prueba automática del mecanismo de Higgs, independientemente de los detalles del potencial. Dado que este punto de vista tiene varias debilidades, por ejemplo la incorporación de las correcciones radiativas cuánticas, enventualmente fue abandonado. Sin embargo, causó un renovado interés en las ideas de Snyder acerca de la no conmutatividad del espacio tiempo.

Otra motivación de las teorías no conmutativas que las mantuvo con cierta vigencia está relacionada con la idea de que, en una teoría cuántica que incluya la gravedad, la naturaleza del espacio tiempo debe cambiar a distancias comparables con la longitud de Plank. El impulso y la energía requeridos para realizar una medida a estas escalas, modificaría por sí mismo la geometría del espacio tiempo [15]. Una manera de formular matemáticamente esto es postular que, a escalas menores que la escala de Plank, el espacio tiempo no es una variedad diferenciable, sino que tiene la estructura de un espacio tiempo no conmutativo. Entonces, una teoría cuántica de la gravitación que contenga o prediga coordenadas no conmutativas, parece tener buenas chances de estar regulada intrínsecamente. Una motivación 
relacionada es que cualquier teoría de la gravedad cuántica no será local en un sentido convencional. La no localidad implica problemas prácticos y conceptuales que aún no han sido entendidos en su totalidad, por lo que las teorías no conmutativas proporcionan un laboratorio relativamente simple donde estudiarlos. Esta es la razón fundamental de la gran actividad reciente en el areas de teoría de campos no conmutativa.

El principal candidato para una teoría cuántica de la gravitación es la teoría de cuerdas, la cual no es local en ningún sentido preciso. De hecho hay más de un parámetro característico de esta no localidad, en general controlada por la mayor entre la longitud de Plank y la longitud $l_{s}$ de las cuerdas fundamentales.

Las teorías de cuerdas han sugerido la posibilidad de un espacio tiempo no conmutativo ya desde los 80, en los trabajos de E. Witten acerca de las teorías de campos de cuerdas [16]. Más recientemente, A. Connes, M. Douglas y A. Schwarz, en [17], M. Douglas y C. Hull en [18] y N. Seiberg y E. Witten en [19], descubrieron que existen límites de bajas energías de la teoría de cuerdas y de la denominada teoría $\mathrm{M}$, que llevan directamente a teorías de gauge no conmutativas y que, siendo mucho mas simples que la teoría de cuerdas original, preservan algo de su no localidad. Probablemente el ejemplo más famoso de este tipo de límites es el desarrollado por Seiberg y Witten en [19]. En este modelo, se estudian cuerdas en presencia de un campo tensorial antisimétrico de Neveu-Schwarz $B_{\mu \nu}$, constante, sobre las cuales se imponen condiciones de contorno de Dirichlet en $p$ direcciones. Estas condiciones de contorno equivalen a restringir el extremo de la cuerda a moverse sobre un hiperplano $p$ dimensional llamado $D p$-brana. En este contexto se verifica que la teoría de campos efectiva, que describe los grados de libertad de baja energía sobre la $D p$-brana, es una teoría no conmutativa $p$-dimensional, donde el conmutador de las coordenadas está relacionado con el campo $B_{\mu \nu}$.

También las teorías de campos no conmutativas juegan un papel importante en el área de la materia condensada. Un ejemplo clásico es el de la teoría de electrones en un campo magnético externo, proyectados sobre el nivel de Landau más bajo, que puede ser tratado como una teoría no conmutativa. Es por esto que estas ideas son relevantes para el estudio del efecto Hall cuántico [20], y de hecho se han demostrado muy útiles en este marco [21].

La mayor parte de estos trabajos relacionados con materia condensada, estuvieron orientados a electrones no interactuantes, por lo que la introducción en este contexto de ideas de la teoría de campos puede contribuir a su progreso. En particular, L. Susskind ha sugerido que la teoría de Chern-Simons definida sobre un espacio no conmutativo puede ser útil para el estudio del efecto Hall [22]. Esta sugerencia se fundamenta en el enfoque lagrangiano del problema de un fluido cargado en un campo magnético intenso, donde la acción natural para describir la dinámica del fluido es una acción de Chern-Simons no conmutativa, pero truncada al primer orden en el desarrollo en serie de potencias del conmutador de las coordenadas. La introducción de los órdenes restantes se justifica apelando al volumen finito del electrón, que se pretende modelar con la celda de Plank. 
En lo que concierne al aspecto puramente matemático, notemos que el marco tradicional de la geometría y la topología es un conjunto de "puntos" con alguna estructura particular que llamamos espacio. Sin embargo, como se descubrió muy tempranamente, aún objetos tan fundamentales como las curvas elípticas se estudian mejor no en términos del conjunto de puntos, sino examinando las funciones continuas que se pueden definir sobre éste. Weierstrass abrió un nuevo camino en la geometría, al estudiar directamente el conjunto de funciones complejas que satisfacen un álgebra de adición particular, y derivar el conjunto de puntos a partir de éstas.

En la geometría no conmutativa, esta idea general de reemplazar conjuntos de puntos por álgebras de funciones se amplía. En muchos casos el conjunto está completamente determinado por el álgebra de funciones, puede entonces abandonarse el conjunto y obtenerse toda la información a partir de las funciones solamente. Por otro lado, en muchos casos el conjunto de puntos es muy patológico, y un examen directo no proporciona información útil. En tales casos, cuando estudiamos el problema desde el punto de vista algebraico, es común encontrar que el álgebra de funciones contiene toda la información que necesitamos. Sin embargo, este álgebra es en general no conmutativa. Entonces el proceso consiste en primero descubrir de que manera las álgebras de funciones determinan la estructura de un conjunto de puntos, y luego determinar cuales son las propiedades relevantes de estas álgebras que no dependen de la conmutatividad. Hecho esto, estamos en condiciones de estudiar la "geometría no conmutativa" generada por un álgebra no conmutativa arbitraria. Fue von Neumann el primero en intentar describir rigurosamente tales "espacios cuánticos", llamando a su estudio "geometría sin puntos".

Las ideas de la geometría no conmutativa fueron revividas en los 80 por los matemáticos Connes y Woronovics, quienes generalizaron la noción de una estructura diferencial al caso no conmutativo, es decir a álgebras arbitrarias. Junto con la definición de una integración generalizada, esto llevó a la descripción algebraica del "espacio tiempo no conmutativo", y permitió la definición de teorías de campos en tales espacios.

En resumen, las teorías no conmutativas constituyen una herramienta de gran utilidad en física teórica. Aparecen tanto en la física de altas energías, para la descripción desde un nivel fundamental del espacio tiempo a pequeña escala, como en el área de materia condensada como modelos efectivos para la descripción del efecto Hall. La enorme actividad actual alrededor de estas teorías está principalmente ligada a la aparición de la no conmutatividad en los límites de bajas energías de la teoría de cuerdas que hemos mencionado anteriormente. Dado que la teoría de cuerdas es la única teoría conocida que podría unificar todas las interacciones fundamentales, es posible que los problemas del control de las divergencias en la teoría cuántica de campos y de la cuantificación de la gravedad estén en última instancia íntimamente relacionados a través de algún tipo de no conmutatividad.

Esta tesis doctoral se inscribe dentro del contexto que hemos descripto en los párrafos precedentes. Estudiaremos en ella varias propiedades de dos teorías de campos de gauge 
de gran interés definidas en espacio no conmutativo: la teoría de Chern-Simons y la teoría de Born-Infeld. Además utilizaremos la acción de Chern-Simons para definir una teoría no conmutativa de la gravitación en tres dimensiones.

En el caso conmutativo, la teoría de Chern-Simons ha tenido innumerables aplicaciones que van desde el cálculo de invariantes (polinomios de Jones) al estudio de teorías conformes, del efecto Hall fraccionario, de superconductores a altas temperaturas y de gravitación [23][36].

La teoría de Chern-Simons ordinaria se puede obtener como la anomalía de paridad en la acción efectiva para fermiones en $2+1$ dimensiones espacio temporales. En el caso no conmutativo, la ruptura de la simetría de Lorentz involucrada en el conmutador de las coordenadas podría generar términos adicionales en la parte que viola paridad de la acción efectiva. Es por lo tanto de interés verificar si en este caso la teoría efectiva coincide con la teoría de Chern-Simons no conmutativa. Por otro lado, como se explicará en los capítulos que siguen, es posible que el límite conmutativo no se pueda intercambiar con el límite de las regularizaciones introducidas en el cálculo de la acción efectiva, en un fenómeno conocido como mezcla infrarrojo-ultravioleta o IR/UV. De esta manera, modelos fermiónicos en los cuales el acoplamiento es proporcional a la no conmutatividad $\left[x^{\mu}, x^{\nu}\right]$, generarán en la acción efectiva, efectos observables independientemente de la magnitud de ésta. Estos problemas son estudiados en el capítulo 6 de esta tesis.

Como hemos dicho antes, la teoría de Chern-Simons no conmutativa se revela de utilidad en el área de materia condensada para el estudio del efecto Hall. Si bien la propuesta original se limita al problema del fluido cargado moviéndose en un plano infinito, dado que las muestras Hall que se estudian en el laboratorio tienen extensión finita, es necesario estudiar el comportamiento de esta acción cuando está definida sobre variedades con borde. En particular es fundamental verificar bajo cuáles condiciones la acción así definida es diferenciable e invariante de gauge. Además es de importancia encontrar qué relación, si la hay, tiene este modelo con el correspondiente modelo conmutativo. Esto se investigará en los capítulos 7 y 8.

Mencionaremos también que, en el caso conmutativo existe una relación entre la gravitación en $2+1$ dimensiones espacio temporales y la teoría de Chern-Simons [35],[36]. Esto sugiere la posibilidad de definir una teoría consistente de la gravitación no conmutativa tridimensional, a partir de la teoría de Chern-Simons no conmutativa. En el caso conmutativo ordinario se conocen soluciones de agujero negro puramente topológicas a las ecuaciones de la gravitación en tres dimensiones [37]. Es de interés verificar si estas soluciones existen en la teoría así definida en el espacio no conmutativo. Estos temas se desarrollan en el capítulo 9 .

En cuanto a la acción de Born-Infeld, ha despertado gran interés recientemente dada su conexión con teoría de cuerdas [42]-[52]. Su definición en el caso no conmutativo enfrenta problemas relacionados con el ordenamiento de los campos, cuando se define este lagrangiano no lineal a partir de su desarrollo en serie de potencias. Desde el punto de vista de la teoría 
de cuerdas, en el problema similar de la acción no abeliana, se ha sugerido un tipo especial de orden simétrico [44] para resolver esta ambigüedad. Es entonces un punto primordial verificar si se puede definir una acción de Born-Infeld no conmutativa que sea invariante de gauge utilizando alguna prescripción de ordenamiento para los campos. Esto lo haremos en el capítulo 10.

Además, en el caso conmutativo no abeliano se ha demostrado que la definición de orden simétrico es consistente con la supersimetría [53]. Se plantea entonces el problema de si es posible encontrar una extensión supersimétrica para la acción de Born-Infeld no conmutativa con ese tipo de ordenamiento. Este problema se estudia en el capítulo 11.

Finalmente en la conclusiones resumiremos nuestros principales resultados, señalando sus alcances y limitaciones. 


\section{Parte I}

\section{Una introducción a las teorías de campos no conmutativas}



En esta primera parte de la tesis, daremos una introducción general a las teorías no conmutativas en matemática y física.

Comenzaremos explicando someramente el marco matemático general en el que se inscriben los espacios no conmutativos y daremos algunas ideas generales acerca de las teorías de campos en estos espacios (capítulo 2).

Completaremos explicando el origen del interés en las teorías de campos no conmutativas al estudiar el límite de bajas energías de teoría de cuerdas y, en el contexto de materia condensada, su relevancia en el estudio del efecto Hall (capítulo 3).

Finalmente estudiaremos la formulación de las teorías de campos de gauge en espacios no conmutativos y su relación con las teorías conmutativas a través del mapeo de Seiberg y Witten (capítulo 4). 


\title{
Capítulo 2
}

\section{Introducción a las teorías no conmutativas}

\begin{abstract}
En este capítulo presentaremos una introducción a las teorías no conmutativas. Luego de describir brevemente el marco matemático en el que se inscribe la geometría no conmutativa, definiremos uno de los ejemplos mas sencillos de no conmutatividad: la deformación $\theta$. Finalmente explicaremos como se formulan las teorías de campos en espacios $\theta$-deformados, y mencionaremos el fenómeno de mezcla infrarrojo-ultravioleta, característico de estas teorías.
\end{abstract}

\section{1. ¿Qué es la geometría no conmutativa?}

Uno de los conceptos más fundamentales de la geometría es la topología. Sobre un cierto conjunto de puntos $\Omega$, que llamaremos espacio una estructura topológica define la forma más primitiva del concepto de "proximidad", esto es, un punto esta "próximo" a otro si ambos pertenecen al mismo conjunto abierto. Sabemos que la estructura topológica de un espacio se refleja en el conjunto $\mathcal{C}_{0}(\Omega)$ de funciones complejas continuas que se pueden definir sobre él. Con la multiplicación punto a punto, el conjunto $\mathcal{C}_{0}(\Omega)$ adquiere estructura de álgebra conmutativa. Ejemplos sencillos de esto son las funciones definidas sobre el toro o sobre la esfera. En general, la topología de un espacio impone fuertes vínculos sobre el tipo de funciones continuas que se pueden definir globalmente.

Cabe entonces preguntarse cuanto se puede recorrer este camino en el sentido inverso: supongamos que de alguna manera conocemos la estructura del álgebra conmutativa $\mathcal{C}_{0}(\Omega)$ de funciones continuas definidas sobre un conjunto de puntos, ¿podemos, con la información presente en ese álgebra, reconstruir la estructura topológica del espacio $\Omega$ ?. La respuesta es sí y es la principal consecuencia del llamado teorema de Gel'fand-Naîmark. Dicho en otras palabras: dada un álgebra conmutativa $\mathcal{C}$, es posible definir un conjunto de puntos $\Omega$ y una estructura topológica sobre él, tal que el álgebra inicial puede ser identificada con el álgebra de funciones continuas definidas en ese conjunto $\mathcal{C}=\mathcal{C}_{0}(\Omega)$. 
Este resultado abre todo un nuevo panorama, basado en la siguiente pregunta: ¿qué otros aspectos de la geometría de un espacio se pueden deducir a partir de su álgebra de funciones continuas?. Se trata del área de la matemática conocida como geometría algebraica, que se ocupa de describir las propiedades geométricas de un espacio $\Omega$ a partir del álgebra conmutativa de funciones continuas definidas sobre él $\mathcal{C}_{0}(\Omega)$, mediante la identificación de los análogos algebraicos de las diferentes estructuras geométricas.

El primer paso en esa dirección es el que hemos mencionado previamente: la identificación del espacio topológico de partida $\Omega$ con el espacio de caracteres, o sea de las representaciones unidimensionales del álgebra conmutativa $\mathcal{C}_{0}(\Omega)$ dotado de una estructura topológica dada por la construcción de Gel'fand-Naîmark-Siegal. El paso siguiente es la identificación de haces vectoriales definidos sobre el espacio topológico con módulos proyectivos de tipo finito con coeficientes en el álgebra, lo que permite formular en términos algebraicos toda la teoría de formas diferenciales sobre una variedad. El cálculo sobre una variedad se reconstruye en el álgebra con la ayuda de la definición de un triple espectral canónico ${ }^{1}$.

Sin embargo, se puede dar un paso más allá en esta dirección: una vez descubiertas las estructuras que, cuando están definidas sobre un álgebra conmutativa $\mathcal{C}$ tienen un significado geométrico en el correspondiente espacio asociado (por ejemplo, las nombradas en el párrafo anterior), podemos estudiarlas per se en el caso de un álgebra no conmutativa $\mathcal{A}$, para preguntarnos con un espíritu abierto, que tipo de interpretación geométrica puedan tener. Esta es la rama de la matemática conocida como geometría no conmutativa.

La geometría no conmutativa se centra en el estudio de aquéllas propiedades de las álgebras no conmutativas y de las estructuras definidas en base a ellas, que tendrían un significado geométrico en el caso conmutativo. En ese sentido, la geometría no conmutativa es una "geometría sin puntos", ya que no se parte de ningún tipo definido de espacio. Podemos pensar que estamos estudiando las propiedades de un "espacio no conmutativo" sin tener que definirlo formalmente.

¿Que interés puede tener todo lo anterior para los físicos?. La primera observación importante es que los campos $\Phi$ son elementos del álgebra de funciones continuas $\mathcal{C}_{0}(\mathcal{M})$ definidas sobre una cierta variedad espacio temporal $\mathcal{M}$. Dado que por el teorema de Gel'fand-Nâimark la variedad $\mathcal{M}$ se puede recuperar a partir del álgebra $\mathcal{C}_{0}(\mathcal{M})$, cabe plantearse la posibilidad de formular una teoría de campos solamente en términos del álgebra a la cual estos pertenecen, sin hacer ninguna referencia directa al espacio-tiempo. Para hacer tal cosa, necesitamos conocer los análogos algebraicos de derivadas, integrales y demás conceptos utilizados en teoría de campos. Como hemos comentado anteriormente, este es un problema que ha sido ampliamente estudiado por los matemáticos.

Pero si somos capaces de abstraer la variedad espacio temporal para definir nuestra teoría de campos en términos algebraicos, ¿que nos impide hacerlo usando un álgebra general $\mathcal{A}$,

\footnotetext{
${ }^{1}$ Una buena introducción pedagógica al significado y utilidad de todos estos términos técnicos en el área de la geometría no conmutativa se puede encontrar en [54]. Otros lecturas interesantes al respecto se pueden encontrar en [55],[56]. La formulación original de las ideas modernas de la geometría no conmutativa desde un punto de vista matemático se puede ver en [5]
} 
incluso no conmutativa?. Nuestros campos serán entonces elementos de $\mathcal{A}$ con ciertas reglas de conmutación, y en algún sentido estamos estudiando la "teoría de campos en un espacio no conmutativo" 2 .

\subsection{Deformación $\theta$ y el producto * de Moyal}

Uno de los ejemplos más simples de geometría no conmutativa es el de las llamadas deformaciones. Estas consisten esencialmente en tomar el álgebra de funciones continuas $\mathcal{C}_{0}(\mathcal{M})$ sobre una cierta variedad $\mathcal{M}$ y "deformar" el producto, substituyéndolo por otro que verifique

$$
(f * g)(x)=f(x) g(x)+\theta \frac{i}{2}\{f(x), g(x)\}_{P B}+O\left(\theta^{2}\right),
$$

donde $\{\cdot, \cdot\}_{P B}$ representa una cierta estructura de Poisson definida sobre la variedad $\mathcal{M}$, y $\theta$ es un parámetro que controla la deformación, tal que $\theta \rightarrow 0$ es el límite conmutativo. Imponiendo como condición adicional la asociatividad del producto, se determinan los términos superiores de la expresión (2.1), a menos de redefiniciones lineales de las funciones [58]. Por lo tanto el producto estrella arriba definido es único para cada estructura de Poisson.

En lo que sigue nos concentraremos en la estructura de Poisson definida de acuerdo con $\theta\{f, g\}_{P B}=\theta^{\mu \nu} \partial_{\mu} f \partial_{\nu} g$, donde $\theta^{\mu \nu}$ es una matriz constante antisimétrica. Este tipo de deformación se denomina deformación $\theta$. El correspondiente producto estrella, o de Moyal se puede escribir a todos los órdenes como

$$
(\hat{f} * \hat{g})(x)=\left.e^{\frac{i}{2} \theta^{\mu \nu} \partial_{\xi \mu} \partial_{\zeta \nu}} \hat{f}(x+\xi) \hat{g}(x+\zeta)\right|_{\xi=\zeta=0} .
$$

Nótese que este producto difiere del producto normal en una derivada total $\partial_{\mu} \tilde{B}^{\mu}[\hat{f}, \hat{g}]$, cuya forma explícita se da en el apéndice 13. Definiremos el paréntesis de Moyal como el conmutador tomado con este producto

$$
[\hat{f}, \hat{g}]=\hat{f} * \hat{g}-\hat{g} * \hat{f} .
$$

El acento circunflejo sobre las funciones indicará que éstas deben ser multiplicadas usando el producto estrella, es decir que las consideraremos como elementos del algebra deformada sobre la variedad $\mathcal{M}$, que llamaremos $\mathcal{C}_{\theta}(\mathcal{M})$, y no como elementos de $\mathcal{C}_{0}(\mathcal{M})$.

Si consideramos las "funciones coordenada" $\hat{x}^{\mu}$ sobre la variedad, tenemos para su conmutador de Moyal

$$
\left[\hat{x}^{\mu}, \hat{x}^{\nu}\right]=i \theta^{\mu \nu}
$$

que es a lo que nos referimos como espacio no conmutativo. Debido a esto, la deformación $\theta$ nos provee de un tipo sencillo de geometría no conmutativa, en la cual aún tiene algún

\footnotetext{
${ }^{2}$ La teoría de campos no conmutativa en su forma más moderna fue formulada originalmente por A. Connes y puede encontrarse en [5]. Otras introducciones pedagógicas son [57]-[60]
} 
sentido hablar de "puntos" y de "coordenadas". Esto simplifica la tarea de definir operaciones de derivación e integración en este álgebra, lo cual, como veremos en la sección que sigue, ayudará en la definición de una acción para los campos no conmutativos.

Como una manera alternativa de definir la deformación, dada una variedad $\mathcal{M}$ de dimensión $d$, considérense $d$ objetos $\hat{x}^{\mu}$ que se multiplican con un producto asociativo que cumple la regla de conmutación (2.4). Estos objetos son la deformación de las coordenadas $x^{\mu}$ sobre la variedad $\mathcal{M}$.

¿Que sucede con el álgebra de funciones sobre la variedad cuando reemplazamos las coordenadas $x^{\mu}$ por los objetos no conmutativos $\hat{x}^{\mu}$ ?. En la expansión en serie de potencias de una dada función de las variables $x^{\mu}$, es necesario decidir en cada término el orden en el cual se acomodaran los objetos $\hat{x}^{\mu}$. Una manera de hacer esto es definir el así llamado orden de Weil, en el cual el objeto $\hat{f}=W(f)$, asociado a la función $f$, se define según la fórmula

$$
\hat{f}=W(f)=\int \frac{d^{d} k}{(2 \pi)^{d}} e^{-i k_{\mu} \hat{x}^{\mu}} \tilde{f}(k),
$$

donde $\tilde{f}(k)$ es la transformada de Fourier de la función $f$. Es decir que definimos el elemento $\hat{f}$ como la transformada inversa de Fourier de $f$ substituyendo en la exponencial las variables $x^{\mu}$ por las correspondientes variables deformadas $\hat{x}^{\mu}$.

Para ver identificar la estructura de álgebra de los elementos $\hat{f}$ multiplicamos dos de estos objetos "en el mismo punto"

$$
\begin{aligned}
\hat{f} \hat{g} & =\int \frac{d^{d} k}{(2 \pi)^{d}} \frac{d^{d} k^{\prime}}{(2 \pi)^{d}} e^{-i k_{\mu} \hat{x}^{\mu}} e^{-i k_{\mu}^{\prime} \hat{x}^{\mu}} \tilde{f}(k) \tilde{g}\left(k^{\prime}\right)= \\
& =\int \frac{d^{d} k}{(2 \pi)^{d}} \frac{d^{d} k^{\prime}}{(2 \pi)^{d}} e^{-i\left(k_{\mu}+k_{\mu}^{\prime}\right) \hat{x}^{\mu}} e^{-\frac{i}{2} k_{\mu}^{\prime} \theta^{\mu \nu} k_{\nu}} \tilde{f}(k) \tilde{g}\left(k^{\prime}\right)= \\
W(f) W(g) & =W(f * g),
\end{aligned}
$$

donde en la segunda línea hemos usado la formula de Campbell-Haussdorf para el producto de exponenciales de objetos no conmutantes y en la tercera línea hemos identificado el integrando con la transformada de Fourier de $f * g$ (ver apéndice 13). Por lo tanto el álgebra cuyos elementos son los objetos $\hat{f}$ definidos de acuerdo con el orden de Weil es isomorfa al álgebra $\mathcal{C}_{\theta}(\mathcal{M})$, lo que nos da otra manera de introducir el producto de Moyal a través de la deformación de la regla de multiplicación de las coordenadas ${ }^{3}$

La propiedades más usadas del producto de Moyal han sido explicadas en el apéndice 13.

\footnotetext{
${ }^{3}$ Esta definición de producto de Moyal es especialmente útil al estudiar solitones en las teorías de campos $\theta$-deformadas.
} 


\subsection{Teorías de campos con producto estrella}

En una teoría de campos no conmutativa con producto estrella, los campos $\hat{\Phi}$ son elementos del álgebra $\mathcal{C}_{\theta}(\mathcal{M})$ definida anteriormente. Es decir que la manera correcta de multiplicar los campos será con el producto estrella de Moyal (2.2). Para construir una acción $S^{\theta}[\hat{\Phi}]$ para ellos, la receta estándard es tomar una acción conmutativa usual $S[\Phi]$ para los correspondientes campos conmutativos $\Phi$, y reemplazar todos los productos en esta presentes por productos de Moyal ${ }^{4}$.

En cualquier teoría no conmutativa con producto estrella, la parte cuadrática de la acción es la misma funcional de los campos que en la correspondiente teoría conmutativa. Para ver esto supongamos que $\hat{\Phi}_{1}$ y $\hat{\Phi}_{2}$ representan dos campos cualesquiera de la teoría (o derivadas de ellos), entonces

$$
\begin{aligned}
\int_{\mathcal{M}} d^{d} x \hat{\Phi}_{1} * \hat{\Phi}_{2} & =\int_{\mathcal{M}} d^{d} x \hat{\Phi}_{1} \hat{\Phi}_{2}+\int_{\partial \mathcal{M}} d^{d-1} x n_{\mu} \tilde{B}^{\mu}\left[\hat{\Phi}_{1}, \hat{\Phi}_{2}\right]= \\
& =\int_{\mathcal{M}} d^{d} x \hat{\Phi}_{1} \hat{\Phi}_{2},
\end{aligned}
$$

donde $n_{\mu}$ es un vector normal al borde de $\mathcal{M}$ y en la última igualdad, hemos supuesto que las condiciones de contorno son tales que el término de superficie $\tilde{B}_{\mu}\left[\hat{\Phi}_{1}, \hat{\Phi}_{2}\right]$ se anula ${ }^{5}$. La forma explícita del término de superficie se puede ver en el apéndice 13. De la ecuación (2.7) se deduce que los propagadores tendrán su forma usual, idénticos a los de la teoría conmutativa.

Sin embargo, las interacciones cambian por la introducción del producto estrella. Consideremos una interacción polinómica (que puede contener derivadas)

$$
\sum_{n=3}^{L} g_{n} \int_{\mathcal{M}} d^{d} x \hat{\Phi}_{1} * \hat{\Phi}_{2} * \cdots * \hat{\Phi}_{n}
$$

Lo primero que se debe observar en tal interacción es que la presencia de las infinitas derivadas involucradas en el producto estrella la convierte en una interacción no local. Esta no localidad se puede analizar en detalle cuando se escribe el producto estrella en su forma integral (ver apéndice 13).

En espacio de impulsos, la interacción se escribe como

$$
\sum_{n=3}^{L} \frac{g_{n}}{(2 \pi)^{d(n-1)}} \int_{\mathcal{M}} d^{d} p_{1} \cdots d^{d} p_{n} \delta\left(\sum_{i}^{n} p_{i}\right) \tilde{\Phi}_{1}\left(p_{1}\right) \tilde{\Phi}_{2}\left(p_{2}\right) \cdots \tilde{\Phi}_{n}\left(p_{n}\right) e^{-\frac{i}{2} \sum_{i<j}^{n} p_{i \mu} \theta^{\mu \nu} p_{j_{\nu}}}
$$

\footnotetext{
${ }^{4}$ Más adelante veremos que esta receta está justificada desde el punto de vista de teoría de cuerdas.

${ }^{5} \mathrm{El}$ el capítulo 7 analizaremos en detalle las condiciones de contorno adecuadas para el caso de la acción de Chern-Simons.
} 
donde $p_{i}$ es el impulso que fluye en el vértice a través del $i$-ésimo $\hat{\Phi}$. Aquí se puede ver que el vértice de interacción difiere del usual conmutativo en un factor de fase adicional. Esta es la única modificación de la reglas de Feynman.

El vértice (2.9) no es invariante bajo permutaciones arbitrarias de los $p_{i}$ por lo que se debe respetar el orden en el cual las líneas emanan de cada vértice en el diagrama de Feynman. Debido a la conservación del impulso, se puede verificar que el vértice sólo es invariante bajo permutaciones cíclicas de los $p_{i}$.

Llamaremos diagramas planos a aquéllos en los cuales los factores de fase en cada vértice se factorizan del diagrama como un solo factor común, que involucra solamente los impulsos de las líneas externas y se puede extraer fuera de las integrales. Las integrales que resultan del cálculo de estos diagramas son idénticas a las correspondientes al caso conmutativo.

En cambio, los diagramas no planos contienen factores de fase en los integrandos que dependen de los impulsos de las líneas interiores de los diagramas, y que por lo tanto no se pueden factorizar fuera de las integrales. Estos son los responsables del fenómeno llamado mezcla infrarrojo-ultravioleta, ubicuo en las teorías no conmutativas ${ }^{6}$.

Como ejemplo de un cálculo perturbativo en una teoría no conmutativa, tomaremos la teoría escalar con interacción cuártica $(\lambda / 4 !) \hat{\phi} * \hat{\phi} * \hat{\phi} * \hat{\phi}$ en $d=4$ dimensiones espaciotemporales y describiremos resumidamente el cálculo de las correcciones a un loop al propagador [61].

Los diagramas planos contribuirán con

$$
\Pi_{\mathrm{p}}^{(1)}(p)=\frac{1}{3} \int \frac{d^{4} k}{(2 \pi)^{4}} \frac{1}{k^{2}+m^{2}} \sim \Lambda^{2}-m^{2} \ln \left(\frac{\Lambda^{2}}{m^{2}}\right),
$$

donde $\Lambda$ es un cutoff ultravioleta y se ve explícitamente la divergencia cuadrática. Por lo tanto, la relación de incerteza de las coordenadas espacio temporales no ha regulado completamente la teoría, algunas divergencias infrarrojas persisten.

La contribución de los diagramas no planos será

$$
\Pi_{\mathrm{np}}^{(1)}(p)=\frac{1}{6} \int \frac{d^{4} k}{(2 \pi)^{4}} \frac{e^{i k_{\mu} \theta^{\mu \nu} p_{\nu}}}{k^{2}+m^{2}} \propto \frac{m}{\sqrt{p_{\mu} \theta^{\mu}{ }_{\rho} \theta^{\rho \nu} p_{\nu}+\frac{1}{\Lambda^{2}}}} K_{1}\left(m \sqrt{p_{\mu} \theta_{\rho}^{\mu} \theta^{\rho \nu} p_{\nu}+\frac{1}{\Lambda^{2}}}\right) .
$$

Puede verse que el factor oscilante en el integrando ha suprimido la divergencia ultravioleta, de manera que el resultado final es convergente en esta región del espectro. Sin embargo, la

\footnotetext{
${ }^{6}$ La razón para esta nomenclatura es la siguiente: es posible definir una expansión diagramática que automáticamente guarde el orden cíclico de los vértices si cada propagador es representado por una cinta en lugar de una línea en el correspondiente diagrama de Feynmann, asociando a cada borde de la $i$-ésima cinta los impulsos $l_{i}$ y $l_{i+1}$ tales que $l_{i+1}-l_{i}=k_{i}$. Esto es similar a la formulación de 't Hooft de las teorías no abelianas. De esta manera los diagramas se pueden clasificar de acuerdo con su topología, y adquiere sentido hablar de diagramas planos o no planos. La serie perturbativa se transforma en una suma sobre topologías, lo que inmediatamente sugiere una conexión entre las teorías de campos no conmutativas y la teoría de cuerdas.
} 
divergencia aparece ahora como una divergencia en la región infrarroja del impulso externo $p$, como se puede ver desarrollando la función $K_{1}$ para valores pequeños del argumento

$$
\begin{aligned}
\Pi_{\mathrm{np}}^{(1)}(p) & \sim \frac{1}{p_{\mu} \theta_{\rho}^{\mu} \theta^{\rho \nu} p_{\nu}+\frac{1}{\Lambda^{2}}}+m^{2} \ln \left(m^{2}\left(p_{\mu} \theta_{\rho}^{\mu} \theta^{\rho \nu} p_{\nu}+\frac{1}{\Lambda^{2}}\right)\right)= \\
& =\Lambda_{e f f}^{2}-m^{2} \ln \left(\frac{\Lambda_{\text {eff }}^{2}}{m^{2}}\right)
\end{aligned}
$$

donde en la segunda línea hemos definido el cutoff efectivo

$$
\Lambda_{e f f}^{2}=\frac{1}{p_{\mu} \theta_{\rho}^{\mu} \theta^{\rho \nu} p_{\nu}+\frac{1}{\Lambda^{2}}} .
$$

Nótese que en el límite ultravioleta, el diagrama no plano (2.12) es finito: la no conmutatividad ha regulado las divergencias. Sin embargo, el diagrama es divergente en la región infrarroja del impulso externo $p$.

Analicemos esto con mayor profundidad. Tomando el límite ultravioleta $\Lambda \rightarrow \infty$ en la formula para en cutoff efectivo (2.13) vemos que $\Lambda_{\text {eff }}^{2}=1 / p_{\mu} \theta^{\mu}{ }_{\rho} \theta^{\rho \nu} p_{\nu}$. Por lo tanto, el límite infrarrojo de $p$ corresponderá a $\Lambda_{e f f} \rightarrow \infty$ y el diagrama (2.12) será divergente en ese límite. Alternativamente, podemos tomar primero el límite infrarrojo en (2.13), entonces $\Lambda_{\text {eff }}=\Lambda$ y el límite $\Lambda \rightarrow \infty$ corresponderá a $\Lambda_{e f f} \rightarrow \infty$ y la fórmula (2.12) será divergente en el ultravioleta. Entonces podemos decir que las divergencias en la región infrarroja del espectro de $p$ provienen de la región ultravioleta de integración. Este fenómeno se conoce como mezcla infrarrojo-ultravioleta o IR/UV.

Recapitulando: para $\theta^{\mu \nu}$ finito, los diagramas no planos son regulados por la no conmutatividad, siendo finitos en el ultravioleta. Sin embargo, en la región de pequeño momento externo, donde las fases que regulan el diagrama devienen inefectivas, reaparecen las divergencias ultravioletas ahora en la forma de divergencias infrarrojas. Se debe hacer notar que estas divergencias provienen de la región ultravioleta de integración, por lo que son una consecuencia de la dinámica de muy alta energía.

Este efecto aparece para $\theta^{\mu \nu}$ no nulo pero arbitrariamente pequeño, por lo que la teoría cuántica no conmutativa debajo de la escala de no conmutatividad no se parece en nada a la correspondiente teoría conmutativa (por ejemplo, como se ve en (2.12) el propagador de la teoría no conmutativa muestra un complicado comportamiento no local). En ese sentido, podemos decir que el límite $\theta^{\mu \nu} \rightarrow 0$ no conmuta con el límite del cutoff. 


\section{Capítulo 3}

\section{La motivación de las teorías no conmutativas en teoría de cuerdas y en materia condensada}

En este capítulo describiremos algunas de las motivaciones de la teorías no conmutativas con producto de Moyal en la física. En particular recorreremos la celebrada deducción de Seiberg y Witten de la no conmutatividad de las teorías de campos que describen la dinámica de d-branas a bajas energías en un campo de Neveu-Schwarz constante. Luego estudiaremos la la manera en la cual aparece un producto de Moyal en materia condensada, cuando se hace la proyección en el nivel de Landau más bajo.

\subsection{Cuerdas abiertas en un campo de Neveu-Schwarz constante}

En esta sección analizaremos el espacio no conmutativo que emerge cuando se estudian cuerdas abiertas en un campo constante de Neveu-Schwarz, siguiendo el análisis original de $[19]^{1}$. Para esto, consideraremos la acción de la cuerda bosónica propagándose en un fondo de campos $g_{A B}, B_{A B}$, derivaremos las condiciones de contorno adecuadas para ese problema y construiremos el propagador en una hoja de mundo con la topología del disco (que corresponde a un cálculo a nivel árbol en teoría de cuerdas). Utilizando este propagador, analizaremos la dependencia de las amplitudes de dispersión con respecto al campo de NeveuSchartz $B_{A B}$.

\footnotetext{
${ }^{1}$ Un enfoque similar usando técnicas de cuantificación canónica se ha dado en [62]
} 


\section{Ecuaciones de movimiento y condiciones de contorno}

La acción para la cuerda bosónica viene dada por

$$
S=\frac{1}{4 \pi \alpha^{\prime}} \int_{\mathcal{W}} d^{2} \sigma\left(g_{A B}(X) \partial_{\alpha} X^{A} \partial^{\alpha} X^{B}-2 \pi i \alpha^{\prime} B_{A B}(X) \epsilon^{\alpha \beta} \partial_{\alpha} X^{A} \partial_{\beta} X^{B}\right),
$$

donde $\mathcal{W}$ es la hoja de mundo, descripta con coordenadas $\sigma_{\alpha}$ con $\alpha=0,1$. En el primer término, los índices a están contraídos con la métrica euclídea de la hoja de mundo $\eta_{\alpha \beta}=$ $\operatorname{diag}(1,1)^{2}$, mientras que en el segundo término, $\epsilon^{\alpha \beta}$ es el tensor antisimétrico de Levi-Civita bidimensional $\epsilon^{01}=-\epsilon^{10}=1$. Aquí $X^{A}$, con $A=0, \cdots, 9$, son las coordenadas de la cuerda en el espacio-tiempo de 10 dimensiones, que se comportan como campos escalares desde el punto de vista de la hoja de mundo, $g_{A B}$ es la métrica del espacio tiempo y $B_{A B}$ es el campo de Neveu-Schwarz antisimétrico.

Para una configuración con $B_{A B}$ y $g_{A B}$ constantes, integrando por partes en el segundo término se obtiene

$$
S=\frac{1}{4 \pi \alpha^{\prime}} \int_{\mathcal{W}} d^{2} \sigma g_{A B} \partial_{\alpha} X^{A} \partial^{\alpha} X^{B}-\frac{i}{2} \int_{\partial \mathcal{W}} d t n_{\alpha} \epsilon^{\alpha \beta} B_{A B} X^{A} \partial_{\beta} X^{B}
$$

aquí la variable de integración $t$ es una coordenada a lo largo del borde $\partial \mathcal{W}$ de la hoja de mundo y $n_{\alpha}$ un vector normal a ese borde. Para la variación de esta acción se tiene

$$
\begin{aligned}
\delta S & =\frac{1}{2 \pi \alpha^{\prime}} \int_{\mathcal{W}} d^{2} \sigma g_{A B} \partial_{\alpha} X^{A} \partial^{\alpha} \delta X^{B}-i \int_{\partial \mathcal{W}} d t n_{a} \epsilon^{\alpha \beta} B_{A B} \delta X^{A} \partial_{\beta} X^{B}= \\
& =-\frac{1}{2 \pi \alpha^{\prime}}\left(\int_{\mathcal{W}} d^{2} \sigma g_{A B} \partial_{\alpha} \partial^{\alpha} X^{A} \delta X^{B}-\int_{\partial \mathcal{W}} d t n_{\alpha}\left(g_{A B} \partial^{\alpha} X^{A}+2 \pi i \alpha^{\prime} \epsilon^{\alpha \beta} B_{A B} \partial_{\beta} X^{A}\right) \delta X^{B}\right)
\end{aligned}
$$

Por lo tanto, para poder extraer la derivada variacional de la acción $\delta S / \delta X^{A}=g_{A B} \partial_{\alpha} \partial^{\alpha} X^{B}$, del primer término en (3.3), debemos anular el segundo término mediante las condiciones de contorno

$$
\begin{array}{rl}
\left.n_{\alpha}\left(g_{A B} \partial^{\alpha} X^{A}+2 \pi i \alpha^{\prime} \epsilon^{\alpha \beta} B_{A B} \partial_{\beta} X^{A}\right)\right|_{\partial \mathcal{W}}=0 & A, B=0, \cdots, p \equiv \mu, \nu \\
\left.\delta X^{A}\right|_{\partial \mathcal{W}}=0 & A=p+1, \cdots, 9 \equiv u
\end{array}
$$

Las condiciones de contorno (3.5) corresponden a condiciones de tipo Dirichlet para las direcciones $p+1, \cdots, 9$ (es decir a una $D p$-brana colocada en las direcciones $0, \cdots, p)$. En cambio, las condiciones (3.4) corresponden a condiciones mixtas, que interpolan entre tipo Dirichlet y tipo Neumann para las direcciones restantes $0, \cdots, p$ (que se que se extienden a lo largo del volumen de mundo de la $D p$-brana $)^{34}$,

\footnotetext{
${ }^{2}$ Si tomáramos en cambio signatura lorentziana $\eta_{\alpha \beta}=\operatorname{diag}(1,-1)$, omitiríamos el " $i$ " delante del $B_{\mu \nu}$.

${ }^{3}$ En adelante llamaremos $\mu, \nu$ a las direcciones a lo largo de la $D p$-brana $0, \cdots, p$, mientras que $u, v$ serán las direcciones transversales $p+1, \cdots, 9$.

${ }^{4}$ Estas condiciones de contorno no son compatibles con con $X^{\mu}$ real (Si bien con una hoja de mundo lorentziana, las condiciones de contorno correspondientes serían reales). Aún así, la teoría de cuerdas abiertas se puede estudiar con ellas, hallando el propagador y utilizándolo en el cálculo de las funciones de correlación.
} 
Las condiciones de contorno de Dirichlet en (3.5) se pueden reescribir $n_{\alpha} \epsilon^{\alpha \beta} \partial_{\beta} X^{u}=0$ en las direcciones $u=p+1, \cdots, 9$. Reemplazando en la forma (3.2) de la acción, vemos que las componentes $u, v=p+1, \cdots, 9$ del campo $B_{A B}$ se desacoplan de los campos $X^{A}$, por lo que podemos poner $B_{u v}=0$. En lo que sigue supondremos no nulas solamente las componentes $B_{\mu \nu}$ de este campo, con $\mu, \nu=0, \cdots, p$.

Nos concentraremos aquí en la aproximación a orden árbol de la teoría de cuerdas, por lo tanto, la topología de la hoja de mundo $\mathcal{W}$ en la cual trabajaremos será la del disco. Cualquier variedad con esta topología se puede mapear conformemente al semiplano superior, entonces condiciones de contorno (3.4) quedan escritas como

$$
\begin{aligned}
\left.\left(g_{\mu \nu} \partial_{\sigma} X^{\nu}+2 \pi i \alpha^{\prime} B_{\mu \nu} \partial_{\tau} X^{\nu}\right)\right|_{\sigma=0} & =0 \\
\left.\partial_{\tau} X^{u}\right|_{\sigma=0} & =0,
\end{aligned}
$$

donde $\sigma_{\alpha}=\sigma, \tau$ son las coordenadas que parametrizan el semiplano, con $\sigma>0$.

\section{Propagador}

En este punto estamos en condiciones de calcular el propagador para esta teoría, el cual deberá cumplir con las siguientes ecuaciones

$$
\begin{aligned}
\partial_{\alpha} \partial^{\alpha} \Delta^{\mu \nu}\left(\sigma_{\alpha}, \sigma_{\alpha}^{\prime}\right) & =-2 \pi \alpha^{\prime} g^{\mu \nu} \delta\left(\sigma_{\alpha}-\sigma_{\alpha}^{\prime}\right), \\
\left.\left(\partial_{\sigma} \Delta^{\mu \nu}+2 \pi i \alpha^{\prime} B^{\mu}{ }_{\rho} \partial_{\tau} \Delta^{\rho \nu}\right)\right|_{\sigma=0} & =0 \\
\left.\partial_{\tau} \Delta^{u v}\right|_{\sigma=0} & =0 .
\end{aligned}
$$

Como se puede verificar por substitución directa, la solución de la ecuación (3.8) con condiciones de contorno (3.9) es

$$
\begin{aligned}
\Delta^{\mu \nu}\left(\sigma_{\alpha}, \sigma_{\alpha}^{\prime}\right)= & -\alpha^{\prime}\left(g^{\mu \nu} \log \frac{\left|\vec{\sigma}-\vec{\sigma}^{\prime}\right|}{\left|\vec{\sigma}-R \vec{\sigma}^{\prime}\right|}+G^{\mu \nu} \log \left|\vec{\sigma}-R \vec{\sigma}^{\prime}\right|^{2}\right)+ \\
& +\frac{i}{\pi} \theta^{\mu \nu} \operatorname{Arctan}\left(\frac{\tau-\tau^{\prime}}{\sigma+\sigma^{\prime}}\right)+D^{\mu \nu}, \\
\Delta^{u v}\left(\sigma_{\alpha}, \sigma_{\alpha}^{\prime}\right)= & -\alpha^{\prime} g^{u v} \log \frac{\left|\vec{\sigma}-\vec{\sigma}^{\prime}\right|}{\left|\vec{\sigma}-R \vec{\sigma}^{\prime}\right|} .
\end{aligned}
$$

donde $R=\operatorname{diag}(-1,1)$ y hemos definido $G^{\mu \nu}$ y $\theta^{\mu \nu}$ de acuerdo a las partes simétrica y antisimétrica respectivamente del tensor $\left(g+2 \pi \alpha^{\prime} B\right)^{-1}$, es decir

$$
\begin{aligned}
& {\left[\frac{1}{g+2 \pi \alpha^{\prime} B}\right]^{\mu \nu}=G^{\mu \nu}+\frac{1}{2 \pi \alpha^{\prime}} \theta^{\mu \nu}, } \\
\theta^{\mu \nu}= & -\left(2 \pi \alpha^{\prime}\right)^{2}\left(\frac{1}{g+2 \pi \alpha^{\prime} B} B \frac{1}{g-2 \pi \alpha^{\prime} B}\right)^{\mu \nu}, \\
G^{\mu \nu}= & \left(\frac{1}{g+2 \pi \alpha^{\prime} B} g \frac{1}{g-2 \pi \alpha^{\prime} B}\right)^{\mu \nu} \\
G_{\mu \nu}= & g_{\mu \nu}-\left(2 \pi \alpha^{\prime}\right)^{2} B_{\mu \rho} g^{\rho \sigma} B_{\sigma \nu} .
\end{aligned}
$$


Las constantes de integración $D^{\mu \nu}$ en (3.11) pueden depender de $B_{\mu \nu}$ pero son independientes de $\sigma, \tau$; no juegan ningún rol esencial y se pueden fijar con libertad en cualquier valor conveniente. Los primeros dos términos son manifiestamente univaluados. El tercer término no es univaluado, por lo que lo restringimos a tomar valores en el intervalo $[-\pi, \pi]$.

La forma que toma el propagador cuando se lo evalúa en el borde de la hoja de mundo, es decir en $\sigma=\sigma^{\prime}=0$, la cual será necesaria para nuestros cálculos más adelante, es

$$
\begin{aligned}
& \left.\Delta^{\mu \nu}\left(\tau, \tau^{\prime}\right)\right|_{\sigma=\sigma^{\prime}=0}=-\alpha^{\prime} G^{\mu \nu} \log \left(\tau-\tau^{\prime}\right)^{2}+\frac{i}{2} \theta^{\mu \nu} \epsilon\left(\tau-\tau^{\prime}\right), \\
& \left.\Delta^{u v}\left(\tau, \tau^{\prime}\right)\right|_{\sigma=\sigma^{\prime}=0}=0,
\end{aligned}
$$

donde hemos hecho $D^{\mu \nu} \equiv 0$. Señalaremos aquí que $G_{\mu \nu}$ tiene una simple interpretación intuitiva como la métrica efectiva que ven las cuerdas abiertas.

Como lo sugiere la notación, el coeficiente $\theta^{\mu \nu}$ en el propagador estará relacionado con el conmutador de las $p+1$ coordenadas $X^{\mu}$ de la cuerda en un espacio no conmutativo. Esta interpretación se obtiene al calcular este conmutador de operadores en la teoría conforme, usando el comportamiento a cortas distancias del orden temporal, esto es

$$
\begin{aligned}
{\left[X^{\mu}(\tau), X^{\nu}(\tau)\right]=} & \lim _{\epsilon \rightarrow 0} T\left(X^{\mu}(\tau) X^{\nu}(\tau-\epsilon)-X^{\mu}(\tau) X^{\nu}(\tau+\epsilon)\right)= \\
= & \lim _{\epsilon \rightarrow 0} T\left(: X^{\mu}(\tau) X^{\nu}(\tau-\epsilon):-X^{\mu}(\tau) X^{\nu}(\tau+\epsilon):\right. \\
& \left.-\Delta^{\mu \nu}(\tau, \tau-\epsilon)+\Delta^{\mu \nu}(\tau, \tau+\epsilon)\right)= \\
= & i \theta^{\mu \nu} .
\end{aligned}
$$

hemos aprovechado aquí que el orden normal : · :, definido de acuerdo con

$$
: X^{A}\left(\sigma_{\alpha}\right) X^{B}\left(\sigma_{\alpha}^{\prime}\right):=X^{A}\left(\sigma_{\alpha}\right) X^{B}\left(\sigma_{\alpha}^{\prime}\right)+\Delta^{A B}\left(\sigma_{\alpha}, \sigma_{\alpha}^{\prime}\right),
$$

es una función analítica en $\sigma_{\alpha}-\sigma_{\alpha}^{\prime}$, lo cual nos permitió escribir la última línea en (3.14). De esta manera vemos que el conmutador de las coordenadas que se extienden a lo largo del volumen de mundo de la $D p$-brana es no nulo, por lo cual podemos inferir que ésta se convertirá en un espacio no conmutativo.

En lo que sigue, verificaremos que la acción efectiva de campos que reproduce las amplitudes de dispersión de teoría de cuerdas se corresponde con la acción de una teoría $\theta$-deformada.

\section{Orden normal y producto de operadores}

Para calcular amplitudes de dispersión en teoría de cuerdas es necesario conocer la forma de un producto arbitrario de ciertos operadores, que están asociados a los estados dispersados, conocidos como operadores de vértice. 
Tales operadores de vértice, para un estado con impulso $p$, tendrán la forma general

$$
V_{p}\left(\sigma_{\alpha}\right)=: P\left(\partial_{\alpha} X^{A}\left(\sigma_{\alpha}\right), \partial_{\alpha} \partial_{\beta} X^{A}\left(\sigma_{\alpha}\right), \cdots\right) e^{i p \cdot X\left(\sigma_{\alpha}\right)}:
$$

donde $P$ es un polinomio en las derivadas de los campos $X^{A}\left(\sigma_{\alpha}\right)$, que dependerá del estado de la cuerda representado con el operador de vértice. Aquí la definición del orden normal se ha extendido para una funcional arbitraria de $X^{A}\left(\sigma_{\alpha}\right)$ de acuerdo con

$$
: \mathcal{F}[X]:=\exp \left\{\frac{1}{2} \int d^{2} \sigma d^{2} \sigma^{\prime} \Delta^{A B}\left(\sigma_{\alpha}, \sigma_{\alpha}^{\prime}\right) \frac{\delta}{\delta X^{A}\left(\sigma_{\alpha}\right)} \frac{\delta}{\delta X^{B}\left(\sigma_{\alpha}^{\prime}\right)}\right\} \mathcal{F}[X]
$$

lo que implica para un producto de funcionales

$$
: \mathcal{F}[X]:: \mathcal{G}[X]:=\exp \left\{\int d^{2} \sigma d^{2} \sigma^{\prime} \Delta^{A B}\left(\sigma_{\alpha}, \sigma_{\alpha}^{\prime}\right) \frac{\delta_{\mathcal{F}}}{\delta X^{A}\left(\sigma_{\alpha}\right)} \frac{\delta_{\mathcal{G}}}{\delta X^{B}\left(\sigma_{\alpha}^{\prime}\right)}\right\}: \mathcal{F}[X] \mathcal{G}[X]:
$$

Como ejemplo de la fórmula anterior, considérese el producto de dos operadores de vértice exponenciales (asociados al estado de taquión) con impulsos $p$ y $q$, evaluados en el borde de la hoja de mundo

$$
\begin{aligned}
: e^{i p \cdot X(\tau)}:: e^{i q \cdot X\left(\tau^{\prime}\right)}: & =e^{-\Delta^{A B}\left(\tau, \tau^{\prime}\right) p_{A} q_{B}}: e^{i p \cdot X(\tau)} e^{i q \cdot X\left(\tau^{\prime}\right)}:= \\
& =\left(\tau-\tau^{\prime}\right)^{2 \alpha^{\prime} p_{\mu} G^{\mu \nu} p_{\nu}} e^{-\frac{i}{2} p_{\mu} \theta^{\mu \nu} p_{\nu} \epsilon\left(\tau-\tau^{\prime}\right)}: e^{i p \cdot X(\tau)} e^{i q \cdot X\left(\tau^{\prime}\right)}:= \\
& =e^{-\frac{i}{2} p_{\mu} \theta^{\mu \nu} p_{\nu} \epsilon\left(\tau-\tau^{\prime}\right)}: e^{i p \cdot X(\tau)}:: e^{i q \cdot X\left(\tau^{\prime}\right)}:
\end{aligned}
$$

donde $\vdots$ · : es el orden normal calculado según la fórmula (3.17), pero usando el propagador

$$
\left.\Delta_{0}^{\mu \nu}\left(\tau, \tau^{\prime}\right)\right|_{\sigma=\sigma^{\prime}=0}=-\alpha^{\prime} G^{\mu \nu} \log \left(\tau-\tau^{\prime}\right)^{2},
$$

en lugar de (3.11). Este coincide con el propagador "usual" correspondiente al caso $B_{\mu \nu} \equiv 0$, con la única diferencia de que la métrica $G_{\mu \nu}$ aparece en lugar de la métrica $g_{\mu \nu}$.

Por lo tanto, este producto de operadores de vértice se reduce al producto de operadores de vértice del caso $B_{\mu \nu} \equiv 0$, salvo por la substitución de la métrica $g_{\mu \nu}$ por la nueva métrica de cuerdas abiertas $G_{\mu \nu}$ y por la aparición del importante factor de fase $\exp \left(i / 2 p_{\mu} \theta^{\mu \nu} p_{\nu}\right)$. Como veremos más adelante, este factor de fase, que es característico del producto de Moyal escrito en el espacio de impulsos, será el responsable de que la teoría efectiva sea no conmutativa.

En cuanto al producto de operadores de vértice mas generales (asociados a estados excitados de la cuerda), se debe observar que como el segundo término en el propagador contiene $\epsilon\left(\tau-\tau^{\prime}\right)$, para $\tau>\tau^{\prime}$ no contribuirá a las contracciones de derivadas de $X^{\mu}(\tau)$, por lo tanto, se puede ver que un producto arbitrario de operadores de vértice será

$$
\begin{aligned}
& \prod_{i=1}^{k}: P_{i}\left(\partial X^{A}\left(\tau_{i}\right), \partial^{2} X^{A}\left(\tau_{i}\right), \ldots\right) e^{i p^{i} \cdot X\left(\tau_{i}\right)}:= \\
& \quad=e^{-\frac{i}{2} \sum_{i>j} p_{\mu}^{i} \theta^{\mu \nu} p_{\nu}^{j} \epsilon\left(\tau_{i}-\tau_{j}\right)} \prod_{i=1}^{k} \vdots P_{n}\left(\partial X^{A}\left(\tau_{i}\right), \partial^{2} X^{A}\left(\tau_{i}\right), \ldots\right) e^{i p^{i} \cdot X\left(\tau_{i}\right)} \vdots
\end{aligned}
$$


Nuevamente, el producto de operadores de vértice coincide con el usual salvo por el factor de fase dependiente de los impulsos y por la substitución de la métrica $g_{\mu \nu}$ por la métrica $G_{\mu \nu}$. La conservación del impulso asegura que el factor de fase sólo depende del orden cíclico de $\operatorname{los} \tau_{i}$ en el borde del disco.

\section{Amplitudes de dispersión}

Ahora, armados con el propagador en la hoja de mundo y con la forma de un producto arbitrario de operadores de vértice, estamos en condiciones de calcular amplitudes de dispersión a orden árbol en la teoría de cuerdas, de manera de descubrir que tipo de teoría de campos es la adecuada para describir la física a bajas energías en esta $D p$-brana no conmutativa.

Para calcular una amplitud de dispersión en teoría de cuerdas, se toma el valor medio de vacío del producto de los operadores de vértice $V_{p_{i}}^{i}\left(\tau_{i}\right)$ que representan a los estados involucrados, evaluados sobre el borde de la hoja de mundo, y se integra sobre los $\tau_{i}$. Si incluimos factores de Chan-Patton en los extremos de la cuerda, debemos también tomar la traza del producto de las matrices $M_{i}$ que representan estos factores.

Por lo tanto, cuando insertemos el producto (3.21) en un valor medio para calcular una amplitud de dispersión entre estados con un conjunto de partículas con impulsos $p_{i}$ y estados de Chan-Patton $M_{i}$, obtendremos como resultado

$$
\begin{aligned}
& S_{i \rightarrow f}\left(G_{\mu \nu}, \theta^{\mu \nu}\right)=\int \prod_{i=1}^{k} d \tau_{i}\left\langle\prod_{i=1}^{k}: P_{i}\left(\partial X^{A}\left(\tau_{i}\right), \partial^{2} X^{A}\left(\tau_{i}\right), \ldots\right) e^{i p^{i} \cdot X\left(\tau_{i}\right)}:\right\rangle \operatorname{Tr}\left(\prod_{i=1}^{k} M_{i}\right)= \\
& =e^{-\frac{i}{2} \sum_{i>j} p_{\mu}^{i} \theta^{\mu \nu} p_{\nu}^{j}} \int \prod_{i=1}^{k} d \tau_{i}\left\langle\prod_{i=1}^{k} \vdots P_{n}\left(\partial X^{A}\left(\tau_{i}\right), \partial^{2} X^{A}\left(\tau_{i}\right), \ldots\right) e^{i p^{i} \cdot X\left(\tau_{i}\right)} \vdots\right\rangle \operatorname{Tr}\left(\prod_{i=1}^{k} M_{i}\right)= \\
& =e^{-\frac{i}{2} \sum_{i>j} p_{\mu}^{i} \theta^{\mu \nu} p_{\nu}^{j}} S_{i \rightarrow f}\left(G_{\mu \nu}, \theta^{\mu \nu} \equiv 0\right) .
\end{aligned}
$$

Es decir que el efecto de un campo $B_{\mu \nu}$ no nulo en las amplitudes de dispersión consiste en cambiar la métrica $g_{\mu \nu}$ por la métrica de cuerdas abiertas $G_{\mu \nu}$, e introducir en los vértices el factor de fase $\exp \left(-i / 2 \sum_{i>j} p_{\mu}^{i} \theta^{\mu \nu} p_{\nu}^{j}\right)$, que caracteriza a una teoría no conmutativa. Por lo tanto, se ve que la acción efectiva construida para reproducir estas amplitudes de dispersión, contendrá productos estrella en sus vértices.

Para construir la teoría efectiva procedemos como sigue: si $\Phi_{a b}^{i}$ son campos asociados a los estados $i$ de la cuerda con factores de Chan-Patton $a b$, entonces en el caso $B_{\mu \nu}=0$ la acción efectiva consiste en una suma de términos de la forma

$$
\operatorname{Tr} \int d^{d} x \Phi_{i_{1}} \Phi_{i_{2}} \cdots \Phi_{i_{n}}
$$

donde la traza se toma sobre los índices de Chan-Patton de los campos. En el caso $B_{\mu \nu}$ no nulo, y de acuerdo con lo que hemos dicho mas arriba, la acción efectiva consistirá en exactamente los mismos términos, pero con los productos normales reemplazados por productos 
estrella

$$
\operatorname{Tr} \int d^{d} x \Phi_{i_{1}} * \Phi_{i_{2}} * \cdots * \Phi_{i_{n}} .
$$

Esto es consistente con la receta dada en 2.3 para construir una teoría de campos no conmutativa: tomar una acción de campos normal y cambiar productos normales por productos de Moyal, es decir introducir en los vértices el factor de fase que aparece en (3.22).

Límite de teoría de campos

Si bien con el razonamiento anterior hemos logrado dar una descripción simple de la dependencia de la acción efectiva en el campo $B_{\mu \nu}$, la forma del desarrollo de la acción efectiva en potencias de $\alpha^{\prime}$ es al menos tan complicada como en el caso $B_{\mu \nu}=0$.

Por lo tanto, para tener una acción de teoría de campos que sea útil para describir la física de bajas energías de la teoría de cuerdas, debemos tomar el límite $\alpha^{\prime} \rightarrow 0$ desacoplando los efectos de altas energías relacionados con el comportamiento de las cuerdas. Como hemos visto que las cuerdas abiertas son sensibles a los parámetros $G_{\mu \nu}$ y $\theta^{\mu \nu}$, al tomar este límite debemos mantener constantes estos parámetros en lugar de los usuales $g_{\mu \nu}$ y $B_{\mu \nu}$.

Para tomar ese límite en la ecuación (3.12), vemos que para mantener $\theta^{\mu \nu}$ constante es necesario que $g_{\mu \nu}$ tienda a cero como $\alpha^{2}$ y $B_{\mu \nu}$ se mantenga constante, entonces

$$
\begin{aligned}
\theta^{\mu \nu} & =\left(\frac{1}{B}\right)^{\mu \nu}, \\
G^{\mu \nu} & =-\frac{1}{\left(2 \pi \alpha^{\prime}\right)^{2}}\left(\frac{1}{B} g \frac{1}{B}\right)^{\mu \nu}, \\
G_{\mu \nu} & =-\left(2 \pi \alpha^{\prime}\right)^{2} B_{\mu \rho} g^{\rho \sigma} B_{\sigma \nu} .
\end{aligned}
$$

Por lo tanto, es claro que la teoría de campos efectiva, construida para reproducir estas amplitudes de dispersión en un cálculo a orden árbol, es similar a la correspondiente al caso $B_{\mu \nu}=0$, salvo que deberá contener productos Moyal en sus vértices, con el parámetro $\theta^{\mu \nu}$ dado por (3.25), de manera de asegurar la aparición del factor de fase en (3.22).

\section{Campos de gauge}

Cuando ponemos de fondo un campo de gauge, la acción de cuerdas (3.1) se modifica con la adición del término

$$
S_{\text {gauge }}=\int_{\partial \mathcal{W}} d t n_{\alpha} \epsilon^{\alpha \beta} A_{A}(X) \partial_{\beta} X^{A}
$$

Como se puede ver integrando por partes, este término es equivalente a sumar al campo $B_{A B}$ la contribución $F_{A B}[A]$. Alternativamente, podríamos absorber el campo $B_{A B}$ en el campo de gauge mediante un término $(1 / 2) B_{A B} X^{B}$. Esta ambigüedad se fija mediante la condición 
$F_{A B}[A] \rightarrow 0$ cuando $X \rightarrow \infty$, por lo que cualquier constante en $F_{A B}[A]$ se considera parte de $B_{A B}$. Nuevamente las condiciones de contorno de Dirichlet desacoplan las componentes $A_{u}$ del campo de gauge, por lo que podemos fijarlas a cero sin pérdida de generalidad.

La adición de un término de interacción de la forma (3.26) convierte nuestro modelo en un modelo sigma no lineal. Por lo tanto, las interacciones darán origen a la aparición de infinitos en los cálculos, los cuales deberán ser regularizados adecuadamente.

La acción (3.1) con el término (3.26) es invariante bajo la transformación

$$
\delta A_{\mu}=\partial_{\mu} \lambda
$$

bajo la cual (3.26) transforma como una derivada total

$$
\delta S_{\text {gauge }}=\int_{\partial \mathcal{W}} d t \partial_{\mu} \lambda(X) \partial_{t} X^{\mu}=\int_{\partial \mathcal{W}} d t \partial_{t} \lambda(X)=0
$$

Por lo tanto, clásicamente la teoría es invariante respecto de las transformaciones (3.27).

Sin embargo, en la integral funcional evaluada con esta acción, es necesario regularizar los infinitos originados por el producto de operadores en el mismo punto. En un valor medio arbitrario, desarrollando la variación de la exponencial de la acción a primer orden en el campo de gauge $A_{\mu}$ y en $\lambda$, obtenemos

$$
\delta\langle\cdots\rangle=\left\langle\int d \tau: A_{\mu}(X(\tau)) \partial_{\tau} X^{\mu}(\tau): \int d \tau^{\prime}: \partial_{\tau^{\prime}} \lambda\left(X\left(\tau^{\prime}\right)\right): \cdots\right\rangle,
$$

donde hemos mapeado conformemente la hoja de mundo al semiplano superior.

Para regularizar este producto de operadores utilizaremos el método de separación de puntos o point splitting, excluyendo de la región de integración en la segunda integral un pequeño intervalo de ancho $\epsilon$ en torno al punto en donde esta evaluado el operador en la primera integral. Al final del cálculo tomaremos el límite $\epsilon \rightarrow 0$. Entonces

$$
\begin{aligned}
\left.\delta\langle\cdots\rangle\right|^{r e g} & =\lim _{\epsilon \rightarrow 0}\left\langle\int d \tau: A_{\mu}(X(\tau)) \partial_{\tau} X^{\mu}(\tau): \int_{R-[t-\epsilon . t+\epsilon]} d \tau^{\prime}: \partial_{\tau^{\prime}} \lambda\left(X\left(\tau^{\prime}\right)\right): \cdots\right\rangle= \\
& =\left\langle\int d \tau: A_{\mu}(X(\tau)) \partial_{\tau} X^{\mu}(\tau)::(\lambda(X(\tau-\epsilon))-\lambda(X(\tau+\epsilon))): \cdots\right\rangle,(3 .
\end{aligned}
$$

usamos el límite de bajas energías $\alpha^{\prime} \rightarrow 0$ del propagador (3.13) para reescibir la última línea como

$$
\begin{aligned}
\left.\delta\langle\cdots\rangle\right|^{r e g} & =\left\langle\int d \tau: \partial_{\tau} X^{\mu}(\tau)\left(A_{\mu}(X(\tau)) * \lambda(X(\tau-\epsilon))-A_{\mu}(X(\tau)) * \lambda(X(\tau+\epsilon))\right): \cdots\right\rangle= \\
& =\left\langle\int d \tau: \partial_{\tau} X^{\mu}(\tau)\left[A_{\mu}(X(\tau)), \lambda(X(\tau))\right]: \cdots\right\rangle .
\end{aligned}
$$


Por lo tanto la acción adecuadamente regularizada por el método de separación de puntos no será invariante frente a las transformaciones (3.27).

Para encontrar las transformaciones que dejan invariante la acción regularizada, es necesario modificar la regla de transformación (3.27) de modo de cancelar la contribución anómala (3.30). De esta manera, tenemos que la acción regularizada será invariante bajo la nueva ley de transformación

$$
\delta A_{\mu}=\partial_{\mu} \lambda+\left[A_{\mu}, \lambda\right] .
$$

Como veremos más adelante, esta ley de transformación se corresponde con una teoría de gauge $\theta$-deformada. Por lo tanto, la simetría de gauge en la brana será una simetría de gauge no conmutativa.

Si bien hemos desarrollado esta demostración en un desarrollo a primer orden en el campo de gauge $A_{\mu}$, la demostración se puede extender a orden arbitrario. El caso no abeliano se demuestra de manera análoga.

Se debe notar que la deducción de (3.32) depende fuertemente del tipo de regularización utilizado. Si hubiéramos utilizado para regularizar las integrales un método invariante de gauge, como por ejemplo el de Pauli-Villars, nos encotraríamos con que la correcta simetría de gauge es (3.28) en lugar de (3.32). Esto cobrará importancia más adelante, cuando definamos el mapeo de Seiberg y Witten, que relaciona una teoría de gauge no conmutativa con una teoría conmutativa.

En resumen, hemos visto que cuando se estudian cuerdas abiertas en un campo de NeveuSchwarz $B_{\mu \nu}$ constante, con sus extremos fijos a una DP-brana, las coordenadas que describen el volumen de mundo de la $D p$-brana verifican el álgebra de un espacio tiempo no conmutativo $\theta$-deformado. Vimos que existe un límite de baja energía de la teoría de cuerdas, en el cual los grados de libertad efectivos están descriptos por una teoría de campos no conmutativa con producto de Moyal. Además, si incluimos un campo de gauge, verificamos que la simetría de gauge de la teoría de campos efectiva se corresponde con la de una teoría de gauge no conmutativa.

\section{2. $\quad$ El efecto Hall}

\section{Partícula en un campo magnético intenso}

Supongamos una partícula de carga e mínimamente acoplada a un campo electromagnético externo. La parte de interacción de la acción vendrá dada por

$$
\begin{aligned}
S_{\text {int }} & =\int d^{4} x j^{\mu}(x) A_{\mu}(x)= \\
& =e \int d^{4} x \int d \tau \delta\left(x^{\rho}-X^{\rho}(\tau)\right) \frac{d X^{\mu}(\tau)}{d \tau} A_{\mu}(x)=
\end{aligned}
$$




$$
=e \int d t\left(\dot{X}^{i}(t) A_{i}(X(t))+A_{0}(X(t))\right)
$$

donde $X^{\mu}$ son las coordenadas de la partícula en el espacio tiempo, y en la última línea hemos fijado la invarianza de reparametrizaciones poniendo $X^{0}=\tau \equiv t$. Especializando para un campo magnético constante en la dirección 3, tenemos $A_{i}(x)=-(B / 2) \epsilon_{i j} x^{j}$ (con $i=1,2)$ y $A_{3}(x)=A_{0}(x)=0$, entonces

$$
S_{i n t}=\frac{e B}{2} \int d t \epsilon_{i j} X^{i} \dot{X}^{j}
$$

La acción completa para la partícula contendrá también un término cinético, que en el límite no relativista será cuadrático en las velocidades $\dot{X}^{i}$ y proporcional a la masa $m$ de la partícula. Está claro que en el límite de campo magnético fuerte $e B>>m$ podemos despreciar esta parte cinética frente al término (3.34). De esta manera obtenemos un sistema de primer orden en derivadas temporales, cuya acción está dada por (3.34).

Al derivar esta acción para obtener los impulsos canónicos, vemos que aparecen los vínculos de segunda clase

$$
p_{i}+\frac{e B}{2} \epsilon_{i j} X^{j}=0 .
$$

Para cuantificar canónicamente esta teoría, debemos obtener los corchetes de Dirac entre las variables canónicas de acuerdo con estos vínculos. Tales corchetes nos dan para las coordenadas

$$
\left\{X^{i}, X^{j}\right\}_{D B}=-\frac{1}{e B} \epsilon^{i j},
$$

lo que tiene una estructura que nos recuerda a un espacio no conmutativo.

La primera cuantificación de la teoría requiere reemplazar las coordenadas $X^{i}$ de la partícula por operadores $\hat{X}^{i}$ cuyas reglas de conmutación vendrán dadas por $i \hbar$ veces el paréntesis de Dirac, es decir

$$
\left[\hat{X}^{i}, \hat{X}^{j}\right]=-\frac{i \hbar}{e B} \epsilon^{i j}=i \theta^{i j}
$$

donde $\theta^{i j}=-\frac{\hbar}{e B} \epsilon^{i j}$. Por lo tanto, la primera cuantificación de una partícula sometida a un campo magnético externo fuerte proporciona naturalmente una realización de un espacio no conmutativo $\theta$-deformado.

Para definir los operadores $\hat{f}$ que representan en la teoría cuántica a los observables clásicos $f$, es necesario dar un prescripción de ordenamiento de las variables $\hat{X}^{1}, \hat{X}^{2}$ en $\hat{f}(\hat{X})$. Si esta prescripción se hace de acuerdo con el orden de Weil definido en (2.5), entonces el producto de operadores $\hat{f} \hat{g}$ será el operador correspondiente al producto Moyal de las funciones $f * g$. Vemos entonces que el álgebra de observables del sistema de una partícula cargada en un campo electromagnético fuerte es un álgebra $\mathcal{C}_{\theta}(\mathcal{M})$. 


\section{Límite continuo: fluido cargado en un campo magnético intenso}

Estudiaremos aquí el límite continuo de las expresiones anteriores, y su relación con la teoría de Chern-Simons no conmutativa, siguiendo el análisis original de [22]. Si escribimos el equivalente de la acción (3.34) para un sistema de $n$ partículas, en el límite $e B>>m$ obtenemos

$$
S_{i n t}=\frac{1}{2} \int d t \sum_{l=1}^{n} e B \epsilon_{i j} X_{l}^{i} \dot{X}_{l}^{j} .
$$

Particionando el plano en $n$ sectores $\Delta_{l} S$ y multiplicando y dividiendo en la acción anterior por $\Delta_{l} S$ se tiene

$$
S_{i n t}=\frac{1}{2} \int d t \sum_{l=1}^{n} \frac{e B}{\Delta_{l} S} \Delta_{l} S \epsilon_{i j} X_{l}^{i} \dot{X}_{l}^{j},
$$

en el límite continuo $n \rightarrow \infty, \Delta_{l} S \rightarrow 0$, esto se transforma en la siguiente acción de campos

$$
S_{\text {int }}=\frac{e \Phi}{2} \int d t d^{2} y \epsilon_{i j} X^{i}(t, y) \dot{X}^{j}(t, y),
$$

donde $\Phi$ es el flujo magnético, que hemos supuesto constante, y hemos reemplazado el índice de partícula $l$ por un par de variables continuas $\left(y^{1}, y^{2}\right)$. Los campos $X^{i}(t, y)$ se pueden entender como las coordenadas al tiempo $t$ de la partícula que se hallaba en $y^{i}$ en el instante inicial. Por lo tanto la condición inicial será $X^{i}(0, y)=y^{i}$.

El lagrangiano (3.40) es invariante frente a difeomorfismos en las variables $y^{i}$ que preservan el área, es decir que tienen jacobiano unidad. Bajo tales difeomorfismos $X^{\prime i}\left(t, y^{\prime}\right)=$ $X^{i}(t, y)$, lo que implica infinitesimalmente

$$
\begin{aligned}
y^{\prime i} & =y^{i}+f^{i}, \\
X^{\prime i} & =X^{i}-\partial_{j} X^{i} f^{j} .
\end{aligned}
$$

Nótese que la condición de jacobiano unidad $\operatorname{det}\left(\partial_{i} y^{\prime j}\right)=1$ se traduce en $\partial_{i} f^{i}=0$ cuya solución es $f^{i}=\epsilon^{i j} \partial_{j} \lambda$, con $\lambda$ una función arbitraria de $y$. Con esto la ley de transformación se convierte en

$$
\begin{aligned}
y^{\prime i} & =y^{i}+\epsilon^{i j} \partial_{j} \lambda, \\
X^{\prime i} & =X^{i}-\epsilon^{j k} \partial_{j} X^{i} \partial_{k} \lambda .
\end{aligned}
$$

La corriente de Nœether asociada a esta simetría es

$$
\begin{aligned}
j^{0} & =\epsilon_{i j} \epsilon^{k l} \partial_{k} X^{i} \partial_{l} X^{j} \lambda, \\
j^{i} & =0,
\end{aligned}
$$

donde hemos hecho caso omiso de una derivada total que no contribuye a la carga. Por lo tanto, la ley de conservación de esta corriente implica

$$
\partial_{\mu} j^{\mu}=\partial_{0} j^{0}=\partial_{0}\left(\epsilon_{i j} \epsilon^{k l} \partial_{k} X^{i} \partial_{l} X^{j}\right) \lambda=0,
$$


por lo vemos que el generador se conserva $\epsilon_{i j} \epsilon^{k l} \partial_{k} X^{i} \partial_{l} X^{j}=$ cte. Es convencional fijar el valor de esta constante a 1 en ausencia de vórtices, por lo que nos quedamos con el vínculo

$$
\epsilon_{i j} \epsilon^{k l} \partial_{k} X_{i} \partial_{l} X_{j}=1
$$

Si imponemos este vínculo agregando un multiplicador de lagrange $A_{0}$, la acción nos queda escrita como

$$
\begin{aligned}
S & =\frac{e \Phi}{2} \int d t d^{2} y\left(\epsilon_{i j} X^{i} \dot{X}^{j}+A_{0}\left(\epsilon_{i j} \epsilon^{k l} \partial_{k} X^{i} \partial_{l} X^{j}-1\right)\right)= \\
& =\frac{e \Phi}{2} \int d t d^{2} y\left(\epsilon_{i j} X^{i}\left(\dot{X}^{j}+\epsilon^{l k} \partial_{k} A_{0} \partial_{l} X^{j}\right)-A_{0}\right) .
\end{aligned}
$$

Si en la expresión anterior hacemos el cambio de variables

$$
X^{i}=y^{i}+\epsilon^{i j} A_{j},
$$

nos queda, después de un poco de álgebra

$$
S=\frac{e \Phi}{2} \int d t d^{2} y \epsilon^{i j}\left(A_{0}\left(\partial_{i} A_{j}+\epsilon^{k l} \partial_{k} A_{i} \partial_{l} A_{j}\right)-A_{i} \partial_{0} A_{j}\right)
$$

donde hemos despreciado las derivadas totales.

Nótese que es segundo término en el coeficiente de $A_{0}$ se puede escribir como el desarrollo a primer orden de un conmutador de Moyal con parámetro $\theta^{k l}=-\epsilon^{k l} / e B$. Entonces nuestra acción difiere de

$$
S^{\theta}=\frac{e \Phi}{2} \int d t d^{2} y \epsilon^{i j}\left(A_{0}\left(\partial_{i} A_{j}+\left[A_{i}, A_{j}\right]\right)-A_{i} \partial_{0} A_{j}\right)
$$

en un término de orden $\left(\theta^{k l}\right)^{2} \sim 1 /(e B)^{2}$. Por lo tanto, cuando el campo magnético es lo suficientemente intenso, podemos despreciar esa diferencia y utilizar (3.50) como la acción para el campo $A_{i}, A_{0}$. Haciendo esto hemos llegado a una teoría no conmutativa que, como veremos más adelante, se puede identificar con la teoría de Chern-Simons no conmutativa para el campo $A_{\mu}=\left(A_{0}, A_{i}\right)$.

$\mathrm{Al}$ adoptar (3.50) como la acción adecuada para describir el fluido de electrones, estamos aceptando una teoría en la cual las coordenadas del espacio tiempo no conmutan, con lo que tenemos una relación de incerteza del tipo $\Delta y_{1} \Delta y_{2} \geq \theta / 2$, lo que implica un área mínima en el plano $\left(y^{1}, y^{2}\right)$ accesible a las observaciones. Podemos interpretar esto diciendo que hemos recuperado de alguna manera el volumen finito ocupado por el electrón. 


\section{Capítulo 4}

\section{Las teorías de gauge no conmutativas y el mapeo de Seiberg-Witten}

Este capítulo estará dedicado a las teorías de gauge es espacio no conmutativo. Primero estudiaremos de que manera se definen los campos de materia en diferentes representaciones del grupo de gauge. Luego definiremos la conexión y la curvatura no conmutativas. Finalmente describiremos la conexión existente entre teorías de gauge conmutativas y no conmutativas, a través del mapeo de Seiberg y Witten

\subsection{Campos de materia}

Podemos ahora preguntarnos como se construirá una teoría de gauge no conmutativa. Sea $G$ el grupo de gauge, cuya álgebra de Lie es generada por los generadores $J_{a}$, con constantes de estructura $f_{b c}^{a}$. En una teoría de gauge convencional, los campos de materia son vectores $\Phi=\left[\Phi_{a}\right]$ de algún espacio de representación del grupo de gauge, con entradas $\Phi_{a}$ que son funciones continuas sobre la variedad $\mathcal{M}$, es decir $\Phi_{a} \in \mathcal{C}_{0}(\mathcal{M})$. Similarmente las transformaciones de gauge son matrices $g=\left[g_{a b}\right]$, que están en alguna representación del grupo de gauge, y cuyas entradas perteneces al álgebra de funciones continuas sobre la variedad, es decir $g_{a b} \in \mathcal{C}_{0}(\mathcal{M})$. Formulamos la teoría de manera que sea invariante frente a $\Phi \rightarrow g \Phi$.

Siguiendo los lineamentos generales descriptos en los capítulos anteriores, debemos reemplazar los campos conmutativos $\Phi_{a} \in \mathcal{C}_{0}(\mathcal{M})$ por campos no conmutativos $\hat{\Phi}_{a} \in \mathcal{C}_{\theta}(\mathcal{M})$. Sin embargo, tal reemplazo implica un cambio en las reglas de transformación frente a cambios de gauge, ya que para poder ser multiplicadas por los campos $\hat{\Phi}_{a}$ (que sólo admiten producto estrella), las entradas $\hat{g}_{a b}$ de las matrices de transformación de gauge deberán pertenecer a $\mathcal{C}_{\theta}(\mathcal{M})$. O sea que la regla correcta será

$$
\hat{\Phi} \rightarrow \hat{g} * \hat{\Phi}
$$


y debemos formular nuestra teoría de manera que sea invariante bajo esta regla de transformación.

En este punto se debe observar una propiedad importante de las teorías de gauge no conmutativas. En una teoría de gauge usual conmutativa, tanto la representación transpuesta o antifundamental, en la cual representamos el campo $\Phi$ por un vector fila y transformamos de acuerdo con $\Phi \rightarrow \Phi g^{-1}$, como la representación adjunta, en la cual pensamos a $\Phi$ como una matriz en el álgebra de Lie del grupo y transformamos según $\Phi \rightarrow g \Phi g^{-1}$, son equivalentes a una representación natural, donde $\Phi \rightarrow g \Phi$ con una adecuada elección de $g$ y $\Phi$. En cambio, en el presente caso, debido a la no conmutatividad del producto estrella, ninguna de esas representaciones es equivalente a alguna forma de (4.1). Entonces, las posibles reglas de transformación para los campos de materia serán

$$
\hat{\Phi}(x) \rightarrow \begin{cases}\hat{g} * \hat{\Phi} & \text { representación fundamental "f", } \\ \hat{\Phi} * \hat{g}^{-1} & \text { representación antifundamental " } a ", \\ \hat{g} * \hat{\Phi} * \hat{g}^{-1} & \text { representación adjunta "adj". }\end{cases}
$$

La inversa $\hat{g}^{-1}$ de una transformación de gauge está definida con relación al producto de Moyal, esto es $\hat{g}^{-1} * \hat{g}=1$. Obsérvese que, aún en el caso con grupo de gauge $U(1)$, tiene sentido diferenciar las tres posibilidades arriba mencionadas para la regla de transformación.

Si escribimos $\hat{g}=1+\hat{\lambda}$, donde $\hat{\lambda}$ es una matriz del álgebra de Lie del grupo con entradas en $\mathcal{C}_{\theta}(\mathcal{M})$, obtenemos la forma infinitesimal de estas transformaciones

$$
\delta \hat{\Phi}(x)= \begin{cases}\hat{\lambda} * \hat{\Phi} & \text { "f", } \\ \hat{\Phi} * \hat{\lambda} & " a " \\ \hat{\lambda} * \hat{\Phi}-\hat{\Phi} * \hat{\lambda} & " a d j "\end{cases}
$$

Por aplicación sucesiva de transformaciones infinitesimales construimos una transformación finita

$$
\begin{aligned}
\hat{g}(x) & =(1+\hat{\lambda}) *(1+\hat{\lambda}) * \cdots *(1+\hat{\lambda})= \\
& =1+\hat{\lambda}+\frac{1}{2} \hat{\lambda} * \hat{\lambda}+\cdots \equiv e_{*}^{\hat{\lambda}}, \\
g^{-1} & =e_{*}^{-\lambda} .
\end{aligned}
$$

\subsection{Conexión, curvatura y derivadas covariantes}

Nótese que, dado que el producto estrella satisface la regla de Leibnitz, las derivadas de los campos se transformarán bajo cambio de gauge de manera diferente a los campos mismos, de acuerdo con

$$
\partial_{\mu} \hat{\Phi} \rightarrow\left\{\begin{array}{lc}
\partial_{\mu} \hat{g} * \hat{\Phi}+\hat{g} * \partial_{\mu} \hat{\Phi} & \text { "f", } \\
\partial_{\mu} \hat{\Phi} * \hat{g}^{-1}+\hat{\Phi} * \partial_{\mu} \hat{g}^{-1} & \text { "a", } \\
\partial_{\mu} \hat{g} * \hat{\Phi} * \hat{g}^{-1}+\hat{g} * \partial_{\mu} \hat{\Phi} * \hat{g}^{-1}+\hat{g} * \hat{\Phi} * \partial_{\mu} \hat{g}^{-1} " a d j ",
\end{array}\right.
$$


es decir que si pretendemos formular una teoría invariante bajo transformaciones de gauge locales $^{1}$, debemos definir un campo de gauge y una derivada covariante. Obsérvese que debido a que en (4.6) están involucrados productos estrella, el campo de gauge no conmutativo $\hat{A}_{\mu}$ debe multiplicarse usando ese producto, tanto en la derivada covariante cuanto en su regla de transformación de gauge.

Si definimos para $\hat{A}_{\mu}$ la regla de transformación

$$
\hat{A}_{\mu} \rightarrow \hat{g} * \hat{A}_{\mu} * \hat{g}^{-1}+\hat{g} * \partial_{\mu} \hat{g}^{-1}
$$

deducimos que las correctas derivadas covariantes adecuadas a cada representación deben ser

$$
\hat{D}_{\mu} \hat{\Phi}= \begin{cases}\partial_{\mu} \hat{\Phi}+\hat{A}_{\mu} * \hat{\Phi} & \text { "f", } \\ \partial_{\mu} \hat{\Phi}-\hat{\Phi} * \hat{A}_{\mu} & \text { "a", } \\ \partial_{\mu} \hat{\Phi}+\left[\hat{A}_{\mu}, \hat{\Phi}\right] & \text { " } a d j "\end{cases}
$$

las cuales transformarán de acuerdo con

$$
\hat{D}_{\mu} \hat{\Phi} \rightarrow \begin{cases}\hat{g} * \hat{D}_{\mu} \hat{\Phi} & \text { "f", } \\ \hat{D}_{\mu} \hat{\Phi}^{*} \hat{g}^{-1} & \text { "a" } \\ \hat{g} * \hat{D}_{\mu} \Phi * \hat{g}^{-1} & \text { "adj"} .\end{cases}
$$

Una observación importante: tomemos la representación adjunta en el caso $U(1)$. Si bien este modelo se desacopla en el límite conmutativo $\theta^{\mu \nu} \rightarrow 0$, para $\theta^{\mu \nu}$ finito la interacción es no trivial como se puede ver en (4.8). Más aún, cuando se consideran los efectos cuánticos, la presencia de $\theta^{\mu \nu}$ arbitrariamente pequeño pero no nulo genera efectos observables cualitativamente diferentes a los del caso estrictamente conmutativo. Esto sucede debido a que, cuando $\theta^{\mu \nu}$ es no nulo, cualquier regularización invariante de gauge hace uso de la derivada covariante (4.8). Otra manera de decir esto es que el límite conmutativo no es un límite suave y no conmuta con los límites tomados al regular las integrales en la teoría de campos. Este fenómeno está cercanamente relacionado con la mezcla infrarrojo-ultravioleta ó IR/UV, de la que hemos hablado anteriormente, que es característica de las teorías no conmutativas y ha sido ampliamente estudiada en la literatura ${ }^{2}$. La forma de la regla de transformación de $\hat{A}_{\mu}$ bajo el cambio infinitesimal (4.3) es

$$
\delta \hat{A}_{\mu}=\hat{D}_{\mu} \hat{\lambda}
$$

\footnotetext{
${ }^{1}$ La palabra "local" debe interpretarse aquí en el sentido débil de "matrices de transformación que dependen de las coordenadas". Las leyes de transformación de gauge (4.2) involucran infinitas derivadas de los campos y de las matrices de transformación, por que son claramente no locales, dependiendo de los valores que toman estas funciones en todo el espacio.

${ }^{2}$ En el capítulo (6) veremos un ejemplo claro de este fenómeno, en el caso de la inducción de un término de Chern-Simons por fluctuación de fermiones no conmutativos en la representación adjunta.
} 
donde $\hat{D}_{\mu}$ corresponde a la representación adjunta. Escrito en componentes

$$
\delta \hat{A}_{\mu}^{a}=\partial_{\mu} \hat{\lambda}^{a}+\frac{1}{2}\left[t_{b}, t_{c}\right]\left\{\hat{A}_{\mu}^{b}, \hat{\lambda}^{c}\right\}+\frac{1}{2}\left\{t_{b}, t_{c}\right\}\left[\hat{A}_{\mu}^{b}, \hat{\lambda}^{c}\right]
$$

En esta fórmula se ve explícitamente una restricción importante para las teorías de gauge no conmutativas: si el campo de gauge $\hat{A}_{\mu}$ debe ser interpretado como una conexión en el álgebra de Lie del grupo de gauge $G$, es necesario que el anticonmutador en el último término sea expresable como combinación lineal de los generadores. Es decir que los grupos admisibles como grupos de gauge de teorías no conmutativas deben ser tales que su álgebra de Lie cierre por anticonmutación (además de por conmutación, como es usual).

Esto nos prohíbe, por ejemplo, los grupos $S O(N), S U(N), S L(N, \mathbb{R}), S L(N, \mathbb{C})$, etc, los cuales deben ser substituidos por grupos cuya álgebra de Lie cierre por anticonmutación (como por ejemplo $U(N), G L(N, \mathbb{C})$ ), etc $)^{3}$.

Con la ayuda de las derivadas covariantes antes definidas, encontramos una expresión para la curvatura $\hat{F}_{\mu \nu}$ en la teoría de gauge no conmutativa

$$
\hat{F}_{\mu \nu}=\left[\hat{D}_{\mu}, \hat{D}_{\nu}\right]=\partial_{\mu} \hat{A}_{\nu}-\partial_{\nu} \hat{A}_{\mu}+\hat{A}_{\mu} * \hat{A}_{\mu}-\hat{A}_{\nu} * \hat{A}_{\mu}
$$

Nótese que debido a que los dos últimos términos en (4.12) involucran productos de Moyal, no se cancelan aún en el caso $U(1)$, por lo que la electrodinámica no conmutativa es una teoría no lineal.

De la definición (4.12) se deduce la correspondiente ley de transformación

$$
\hat{F}_{\mu \nu} \rightarrow \hat{g} * \hat{F}_{\mu \nu} * \hat{g}^{-1}
$$

o sea que la curvatura transforma en la representación adjunta.

\subsection{Acción invariante de gauge}

La receta general que hemos descrito en el capítulo 2.3 para construir la acción de una teoría no conmutativa consiste en tomar la acción de alguna teoría de campos conmutativa y reemplazar en ella los campos $\Phi$ por los correspondientes campos no conmutativos $\hat{\Phi}$ y los productos usuales por productos de Moyal. Apliquemos esta receta para los campos de una teoría de gauge

\footnotetext{
${ }^{3}$ Sin embargo, es posible sortear este inconveniente si se renuncia a la interpretación del campo de gauge como una conexión, o si se imponen vínculos adicionales sobre el campo. De esta manera se han construido ejemplos de teorías de gauge con grupos especiales o simplécticos [63], [64], [65].
} 


\section{Representaciones fundamental y antifundamental}

Para un campo escalar $\phi$ en la representación fundamental (antifundamental), tomamos la acción de la teoría conmutativa y le aplicamos la regla anterior para obtener

$$
S^{\theta}=\frac{1}{2} \int d^{d} x\left(\left(\hat{D}^{\mu} \hat{\phi}\right)^{\dagger} * \hat{D}_{\mu} \hat{\phi}-m^{2} \hat{\phi}^{\dagger} * \hat{\phi}\right)
$$

donde $\hat{D}_{\mu} \phi$ es la derivada covariante en la representación fundamental (antifundamental). Para referencia futura, nótese que obtenemos el mismo resultado si aplicamos nuestra regla de reemplazo a la derivada covariante escrita en su forma matricial $D_{\mu} \phi=\partial_{\mu} \phi+A_{\mu} \phi$ o en términos de componentes $\left(D_{\mu} \phi\right)^{a}=\partial_{\mu} \phi^{a}+f_{b c}^{a} A_{\mu}^{b} \phi^{c}$.

De manera similar, para un campo espinorial en la representación fundamental (antifundamental) se tiene

$$
S^{\theta}=\frac{1}{2} \int d^{d} x \overline{\hat{\psi}} *(i \hat{\not D}-m) \hat{\psi} .
$$

Tanto en el caso fermiónico como en el bosónico el lagrangiano para los campos en la representación fundamental (antifundamental) es invariante de gauge, y por lo tanto lo mismo sucede con la acción.

\section{Representación adjunta}

En el caso de que los campos de materia están en la representación adjunta (4.2), la acción invariante de gauge será, para un campo escalar

$$
S^{\theta}=\frac{1}{2} \operatorname{Tr} \int d^{d} x\left(\left(\hat{D}^{\mu} \hat{\phi}\right)^{\dagger} * \hat{D}_{\mu} \hat{\phi}-m^{2} \hat{\phi}^{\dagger} * \hat{\phi}\right)
$$

mientras que para un campo espinorial

$$
S^{\theta}=\frac{1}{2} \operatorname{Tr} \int d^{d} x(\overline{\hat{\psi}} *(i \hat{D}-m) \hat{\psi}),
$$

donde en ambos casos la derivada covariante es la correspondiente a la representación adjunta según se define en (4.8).

Debemos hacer aquí una observación importante: las expresiones (4.16) y (4.17) se obtienen a partir de la correspondiente teoría conmutativa aplicando la receta de la que hablamos anteriormente (esto es, reemplazando los campos conmutativos por los no conmutativos y los productos usuales conmutativos por productos estrella) a las formas matriciales de las derivadas covariantes $D_{\mu} \phi=\partial_{\mu} \phi+\left[A_{\mu}, \phi\right]$. Otro hubiera sido el resultado de aplicar nuestra receta a la forma en componentes de la derivada covariante $\left(D_{\mu} \phi\right)^{a}=\partial_{\mu} \phi^{a}+f_{b c}^{a} A_{\mu}^{b} \phi^{c}$. Esta observación cobra importancia al construir la acción para el campo de gauge. 
Se debe hacer notar que, tanto el lagrangiano en (4.16) como aquél en (4.17), para los campos de materia en la representación adjunta, no son invariantes de gauge sino que transforman según

$$
\mathcal{L}=\operatorname{Tr}(\cdot) \rightarrow \operatorname{Tr}\left(g^{-1} * \cdot * g\right),
$$

el producto de Moyal en la expresión resultante nos impide usar la propiedad cíclica de la traza para probar la invarianza de gauge del lagrangiano. Sin embargo, si se imponen condiciones de contorno adecuadas, el producto estrella cumple la propiedad cíclica bajo el signo integral, por lo tanto la acción construída con este lagrangiano será invariante de gauge.

\section{Acción para el campo de gauge}

Cuando nos disponemos a aplicar nuestra receta para escribir la acción invariante de gauge para los campos de gauge $A_{\mu}$, tomamos una acción conmutativa usual, la escribimos en su forma matricial y recién después reemplazamos los campos por los correspondientes campos no conmutativos $\hat{A}_{\mu}$ y los productos usuales por productos de Moyal. Nuestra receta para construir acciones no conmutativas no se puede aplicar a la acción escrita en términos de componentes $\hat{A}_{\mu}^{a}$.

Por ejemplo, para la acción de Yang-Mills no conmutativa obtenemos

$$
S_{Y M}^{\theta}=\frac{1}{4} \int d^{D} x \operatorname{Tr}\left(\hat{F}_{\mu \nu} * \hat{F}^{\mu \nu}\right) .
$$

Obsérvese que el lagrangiano no es invariante de gauge, pero la acción sí lo es dadas las adecuadas condiciones de contorno. Esto es similar a lo que observamos para los campos de materia en el caso de la representación adjunta. Otra observación importante es que esta acción contiene autointeracción para el campo de gauge aún en el caso $U(1)$, por lo cual el desarrollo perturbativo de la electrodinámica no conmutativa contendrá diagramas con loops de fotones.

En la Parte II de la tesis definiremos la acción de Chern-Simons no conmutativa siguiendo los lineamientos aquí descriptos. En la Parte III haremos lo propio en relación a la acción no conmutativa de Born-Infeld.

\subsection{El mapeo de Seiberg y Witten}

Hemos visto en la sección 3, que al regularizar la integral funcional de teoría de cuerdas usando el método de point splitting se obtiene una teoría de gauge no conmutativa cuyos campos hemos llamado $\hat{A}_{\mu}$, mientras que en cambio, si regularizáramos usando el método de Pauli-Villars, obtenemos una teoría de gauge usual conmutativa con campos de gauge $A_{\mu}$. Por lo tanto, dado que se trata de una diferencia de regularización, debe existir una redefinición de los campos $\hat{A}_{\mu}\left[A_{\mu}\right]$, que mapee la simetría de gauge ordinaria conmutativa en 
la correspondiente simetría no conmutativa. Tal transformación fue encontrada por Seiberg y Witten, en la forma de un cambio de variables orden a orden en $\theta^{\mu \nu}$ [19].

Dado que debemos respetar las simetrías presentes a cada lado de la transformación, esto significa que para un cambio infinitesimal de gauge con parámetro $\lambda$ en la teoría conmutativa, debe existir un $\hat{\lambda}$ en la teoría no conmutativa tal que

$$
\hat{A}_{\mu}\left[A_{\mu}+\delta_{\lambda} A_{\mu}\right]=\hat{A}_{\mu}\left[A_{\mu}\right]+\delta_{\hat{\lambda}} \hat{A}_{\mu}\left[A_{\mu}\right] .
$$

Es importante resaltar que esto no significa que exista un cambio de parámetros del tipo $\hat{\lambda}=\hat{\lambda}[\lambda]$, ya que si así fuera tendríamos un isomorfismo entre los grupos de gauge de ambas teorías. Basta con recordar que en el caso $U(1)$ la teoría no conmutativa es "no abeliana" (a diferencia de la conmutativa), para ver que tal isomorfismo es imposible. Entonces, lo mas cercano que podemos escribir es una transformación de la forma $\hat{\lambda}=\hat{\lambda}\left[\lambda, A_{\mu}\right]$.

Postularemos que, en un desarrollo del campo $\hat{A}_{\mu}\left[A_{\mu}\right]$ en potencias de $\theta^{\mu \nu}$, los coeficientes son expresiones locales en términos del campo $A_{\mu}$ y sus derivadas. Entonces, como se puede verificar por substitución directa, una solución de la condición (4.20) a primer orden en $\theta^{\mu \nu}$ será

$$
\begin{aligned}
& \hat{A}_{\mu}[A]=A_{\mu}+\frac{1}{4} \theta^{\rho \sigma}\left\{A_{\rho}, \partial_{\sigma} A_{\mu}+F_{\sigma \mu}\right\}+O\left(\left(\theta^{\mu \nu}\right)^{2}\right), \\
& \hat{\lambda}[\lambda, A]=\lambda-\frac{1}{4} \theta^{\rho \sigma}\left\{\partial_{\rho} \lambda, A_{\sigma}\right\}+O\left(\left(\theta^{\mu \nu}\right)^{2}\right),
\end{aligned}
$$

(obsérvese que, dado que esta es una expresión a primer orden en $\theta^{\mu \nu}$, los productos involucrados son productos matriciales usuales).

Sin embargo, en este punto debemos notar que en realidad nuestra deducción de la ecuación (4.20) y de su solución (4.21) es independiente del valor de $\theta^{\mu \nu}$. En otras palabras, nuestros razonamientos siguen siendo válidos si el campo de gauge $A_{\mu}$ se refiere a una teoría no conmutativa con parámetro $\theta^{\mu \nu} \neq 0$ y el campo $\hat{A}_{\mu}$ a una teoría no conmutativa con parámetro $\theta^{\mu \nu}+\delta \theta^{\mu \nu} \neq 0$. En ese caso, la solución se puede escribir como

$$
\begin{aligned}
& \delta \hat{A}_{\mu}[A]=\frac{1}{4} \delta \theta^{\rho \sigma}\left\{A_{\rho}, \partial_{\sigma} A_{\mu}+F_{\sigma \mu}\right\}, \\
& \delta \hat{\lambda}[\lambda, A]=-\frac{1}{4} \delta \theta^{\rho \sigma}\left\{\partial_{\rho} \lambda, A_{\sigma}\right\} .
\end{aligned}
$$

La aplicación de este cambio de variables en la acción efectiva para el campo de gauge transforma la acción no conmutativa $S^{\theta}\left[\hat{A}_{\mu}\right]$ en un acción conmutativa $S[A]$, que no necesariamente es aquella de la cual se obtuvo $S^{\theta}\left[\hat{A}_{\mu}\right]$ al reemplazar productos estrella por productos normales. Por ejemplo la aplicación a la acción de Yang-Mills no conmutativa (4.19) del mapeo de Seiberg y Witten no la transforma en la acción de Yang-Mills conmutativa, sino en una acción conmutativa complicada y no polinómica. Estudiaremos más adelante el caso de la acción de Chern-Simons en $2+1$ dimensiones. 
Para referencia futura, señalaremos aquí que la regla de transformación para el tensor de curvatura $\hat{F}_{\mu \nu}$ que se deduce de (4.22) es

$$
\delta \hat{F}_{\mu \nu}=\frac{1}{4} \delta \theta_{\rho \sigma}\left(-2\left\{F_{\mu \rho}, F_{\nu \sigma}\right\}+\left\{A_{\rho}, \partial_{\sigma} F_{\mu \nu}+D_{\sigma} F_{\mu \nu}\right\}\right) .
$$




\section{Parte II}

\section{Teoría de Chern-Simons en espacio no conmutativo}



En esta segunda parte de la tesis, analizaremos algunos aspectos de la teoría de ChernSimons en espacio no conmutativo.

Antes que nada, resumiremos las propiedades de la acción de Chern-Simons conmutativa que nos proponemos estudiar en el caso no conmutativo (capítulo 5).

Comenzaremos el estudio del caso no conmutativo, investigando la manera en la cual la acción de Chern-Simons no conmutativa se induce al integrar fermiones en $2+1$ dimensiones no conmutativas acoplados a un campo de Gauge, como la parte que viola paridad de la acción efectiva (capítulo 6).

A continuación estudiaremos los problemas que surgen al definir la acción de ChernSimons no conmutativa en una región del espacio con borde y la relación entre esta acción y el modelo quiral no conmutativo de Wess-Zumino-Witten en el borde de esa región (capítulo 7).

Como un último punto, estudiaremos el comportamiento de la acción de Chern-Simons bajo la transformación de Seiberg y Witten, la que mapea teorías de gauge no conmutativas en conmutativas, y de la cual hablamos en el capítulo precedente (capítulo 8).

Finalmente, utilizando el conocimiento adquirido en relación con este modelo, daremos una definición consistente de una teoría de gravedad $\theta$-deformada en $2+1$ dimensiones (capítulo 9).

Toda esta sección forma parte de las contribuciones originales de esta tesis, y fue desarrollada en colaboración con F.A. Schaposnik y G.A. Silva (de la Universidad Nacional de La Plata) y M. Bañados y O. Chandía (de la Universidad Católica de Chile). 


\section{Capítulo 5}

\section{Introducción a la teoría de Chern-Simons}

En este capítulo repasaremos algunas propiedades conocidas de la acción de ChernSimons en el espacio conmutativo, con el objeto de estudiarlas mas adelante en el caso no conmutativo. En particular, nos referiremos a la inducción de un término de Chern-Simons por integración de fermiones masivos en $2+1$ dimensiones, la relación de la acción de Chern-Simons definida en una variedad con borde con la acción quiral de Wess-Zumino-Witten, y la formulación de la gravedad tridimensional como una teoría de Chern-Simons. En la última sección, definiremos la acción de Chern-Simons en espacio no conmutativo.

\subsection{La acción de Chern-Simons en espacio conmutati- vo}

\section{Definición}

Cuando definimos una teoría de gauge en tres dimensiones espacio temporales, existe otra estructura que puede suplementar o reemplazar al término de Maxwell o Yang-Mills en la acción para los campos de gauge. Ésta es el término de Chern-Simons [23], el cual se define como

$$
S_{C S}\left[A_{\mu}\right]=\frac{\kappa}{4 \pi} \operatorname{Tr} \int_{\mathcal{M}} d^{3} x \epsilon^{\mu \nu \rho}\left(A_{\mu} \partial_{\nu} A_{\rho}+\frac{2}{3} A_{\mu} A_{\nu} A_{\rho}\right)
$$

donde $\kappa$ es una constante de acoplamiento o nivel, que es adimensional (cuando los campos se toman con las dimensiones adecuadas) y que es una medida de la intensidad con la cual el término de Chern-Simons determina la dinámica de la teoría. Remarcablemente, éste término no depende de la elección de la métrica sobre la variedad espacio temporal $\mathcal{M}$, es decir que se trata de un término topológico, que no dará contribuciones al tensor energía impulso del sistema. 
Este término contribuye a la variación de la acción con

$$
\delta S_{C S}=\frac{\kappa}{4 \pi} \operatorname{Tr} \int_{\mathcal{M}} d^{3} x \epsilon^{\mu \nu \rho} F_{\nu \rho} \delta A_{\mu}+\frac{\kappa}{4 \pi} \operatorname{Tr} \int_{\partial \mathcal{M}} d^{2} x n_{\rho} \epsilon^{\mu \nu \rho} A_{\nu} \delta A_{\mu}
$$

Para garantizar la diferenciabilidad de la acción, debemos anular el término de superficie en (5.2). Para esto es suficiente imponer la condición de contorno $\left.A_{0}\right|_{\partial \mathcal{M}}=0$ (o cualquiera de las componentes espaciales). De esta manera obtenemos una contribución adicional en las ecuaciones de movimiento de valor $(\kappa / 4 \pi) \epsilon^{\mu \nu \rho} F_{\nu \rho}$.

Bajo una transformación de gauge, que debe ser elegida de manera de respetar las condiciones de contorno de las que hablamos en el párrafo anterior, la acción de Chern-Simons no es invariante, sino que cambia de acuerdo con

$$
\delta S_{C S}=-\frac{\kappa}{4 \pi} \operatorname{Tr} \int_{\partial \mathcal{M}} d^{2} x n_{\mu} \epsilon^{\mu \nu \rho} \partial_{\nu} g g^{-1} A_{\rho}-\frac{\kappa}{12 \pi} \operatorname{Tr} \int_{\mathcal{M}} d^{3} x \epsilon^{\mu \nu \rho} g \partial_{\mu} g^{-1} g \partial_{\nu} g^{-1} g \partial_{\rho} g^{-1} .
$$

Mientras que el primer término en (5.3) contiene $A_{\mu}$, y se anula debido a las condiciones de contorno $\left.A_{0}\right|_{\partial \mathcal{M}}=0$, el segundo término es un término de superficie, que es proporcional al "número de enrollamiento" o winding number $W(g)$, esto es al número de veces que se "enrolla" la transformación gauge en la variedad.

Por lo tanto, tenemos que bajo una transformación de gauge, la acción cambia según $\delta S_{C S}=-2 \pi \kappa W(g)$. Como en la teoría cuántica $e^{i S_{C S}}$ debe ser invariante de gauge, vemos que necesariamente el coeficiente $\kappa$ debe estar cuantizado $\kappa \in Z$.

\section{La anomalía de paridad}

Una de las maneras en la que aparece la acción de Chern-Simons en la física, es como acción efectiva por correcciones radiativas que resultan de la integración de fermiones acoplados a un campo de gauge en $2+1$ dimensiones, como la parte que viola paridad de la acción efectiva invariante de gauge para el campo $A_{\mu}[24]$.

Definamos la acción efectiva $\Gamma\left[A_{\mu}\right]$ de acuerdo con

$$
e^{i \Gamma\left[A_{\mu} ; m\right]}=\int \mathcal{D} \psi \mathcal{D} \bar{\psi} e^{i S\left[\psi, A_{\mu} ; m\right]},
$$

donde $S\left[\psi, A_{\mu} ; m\right]$ es la acción para fermiones de masa $m$ mínimamente acoplados al campo $A_{\mu}$ en alguna representación del grupo de gauge.

$\mathrm{Al}$ intentar calcular explícitamente por medio de la teoría de perturbaciones los primeros órdenes de la acción efectiva, nos encontramos con diagramas divergentes, en particular los diagramas de polarización de vacío y del triángulo. Un método invariante de gauge de regular estas divergencias es el de Pauli-Villars, que consiste en agregar a la teoría nuevos campos espinoriales bosónicos de masa $M$, que al final de los cálculos se hace tender a infinito. Pero 
en $d=3$ dimensiones, la introducción de una masa, en este caso la del campo regulador, entraña una violación de la simetría de paridad, y es por esto que el cálculo de los diagramas regularizados de esta manera lleva a un término de Chern-Simons.

Como resultado, la parte de la acción efectiva invariante de gauge que viola paridad tiene la forma de una acción de Chern-Simons

$$
\Gamma_{\text {impar }}\left[A_{\mu} ; m=0\right]=\frac{1}{2} \frac{M}{|M|} S_{C S}\left[A_{\mu}\right] .
$$

Si bien el factor $1 / 2$ delante de la acción efectiva haría pensar que no se respeta la invarianza de gauge frente a transformaciones de gauge grandes, esto es, con winding number $W(g)$ diferente de cero, la variación del término de Chern-Simons bajo estas transformaciones se cancela con la variación de la parte de la acción efectiva que preserva paridad.

En el caso de los fermiones masivos, la violación de paridad está explícita desde el inicio a través del término de masa. Pero además, podemos calcular la parte que viola paridad de la acción efectiva invariante de gauge por efectos radiativos, la cual, a baja energía y orden $e^{3}$ en teoría de perturbaciones, tiene la forma ${ }^{1}$

$$
\Gamma_{\text {impar }}\left[A_{\mu} ; m\right]=\frac{1}{2}\left(\frac{m}{|m|}+\frac{M}{|M|}\right) S_{C S}\left[A_{\mu}\right]+o\left(\frac{\partial^{2}}{m^{2}}, e^{4}\right) .
$$

Nótese que en este caso masivo, el resultado es aproximado, ya que recibe correcciones de la región de altas energías (la forma explícita de estas correcciones, que para el caso abeliano ha sido estudiada en [28],[29], no corresponde a un término de Chern-Simons), además de correcciones de orden $e^{4}$ en teoría de perturbaciones. Por otro lado la parte de Chern-Simons es invariante de gauge aún bajo transformaciones grandes, independientemente de la parte que preserva paridad.

Otros métodos invariantes de gauge para la regularización de las integrales dan idéntica respuesta. Un sumario de estos resultados se puede encontrar en [27]. Si en lugar de utilizar un método invariante de gauge para regular las divergencias, hubiéramos utilizado un método que preserve la simetría de paridad de la acción original, habríamos encontrado que la acción efectiva resultante viola la simetría de gauge.

En cuanto a las correcciones perturbativas, para el caso abeliano [30] como para el no abeliano [31],[32] se ha probado que el coeficiente del término de Chern-Simons no se renormaliza a todo orden en teoría de perturbaciones.

\section{La acción de Chern-Simons en variedades con borde}

Dado que la acción de Chern-Simons tiene un carácter topológico, es de interés estudiar los efectos que tiene sobre esta teoría la topología de la variedad sobre la cual están definidos los campos de gauge [33]-[34].

\footnotetext{
${ }^{1}$ Si bien tanto en la definición de la acción de Chern-Simons como en la acción fermiónica no hemos incuído explícitamente la constante de acoplamiento $e$, ésta puede introducirse mediante el reescaleo $A_{\mu} \rightarrow e A_{\mu}$
} 
Considérese la acción

$$
S_{C S}\left[A_{0}, A_{i}\right]=\frac{\kappa}{4 \pi} \operatorname{Tr} \int_{\mathcal{M}} d^{3} x \epsilon^{i j}\left(A_{0} F_{i j}-A_{i} \partial_{0} A_{j}\right)
$$

la cual difiere de la acción de Chern-Simons usual por un término de superficie. Si $\mathcal{M}$ no tiene borde, tal término de superficie es irrelevante, caso contrario el termino se anula cuando las condiciones de contorno son las mencionadas anteriormente.

Usando la forma (5.7) para la acción, la función de partición para la teoría de ChernSimons toma la forma

$$
\begin{aligned}
Z & =\int \mathcal{D} A_{i} \mathcal{D} A_{0} e^{i S_{C S}\left[A_{0}, A_{i}\right]}= \\
& =\int \mathcal{D} A_{i} \mathcal{D} A_{0} e^{\frac{i \kappa}{4 \pi} \operatorname{Tr} \int_{\mathcal{M}} d^{3} x \epsilon^{i j}\left(A_{0} F_{i j}-A_{i} \partial_{0} A_{j}\right)},
\end{aligned}
$$

entonces vemos que el campo $A_{0}$ actúa como un multiplicador de Lagrange forzando la condición de conexión plana para las componentes espaciales del campo de gauge. Integrando sobre $A_{0}$ nos queda

$$
Z=\int \mathcal{D} A_{i} \delta\left(\epsilon^{i j} F_{i j}\right) e^{-\frac{i \kappa}{4 \pi} \operatorname{Tr} \int_{\mathcal{M}} d^{3} x \epsilon^{i j} A_{i} \partial_{0} A_{j}}
$$

Para seguir adelante con este cálculo, debemos resolver la condición de conexión plana para las componentes espaciales del campo de gauge $A_{i}$. La solución de esta ecuación depende fuertemente de la topología de la variedad $\mathcal{M}$, y el resultado final contendrá un cierto número de grados de libertad, en la forma de variables o funciones arbitrarias, que constituyen lo que se suele llamar el espacio de módulos del problema.

Por ejemplo, si elegimos como nuestra variedad espacio temporal $\mathcal{M}=\Sigma \times \mathbb{R}$ donde $\Sigma$ es una variedad bidimensional con la topología del disco, la solución de la condición de conexión plana es simplemente $A_{i}=g \partial_{i} g^{-1}$, con $g: \mathcal{M} \rightarrow G$. Entonces el espacio de módulos en este caso consiste en las funciones sobre la variedad que toman valores en el grupo de gauge. Reinsertando en (5.9) tenemos

$$
Z=\int \mathcal{D} g e^{i S_{Q W Z W[g]}}
$$

donde $S_{Q W Z W}[g]$ es la acción quiral de Wess-Zumino-Witten

$$
S_{Q W Z W}[g]=-\frac{\kappa}{4 \pi} \operatorname{Tr} \int_{\partial \mathcal{M}} d^{2} x g \partial_{0} g^{-1} g \partial_{\varphi} g^{-1}-\frac{\kappa}{12 \pi} \operatorname{Tr} \int_{\mathcal{M}} d^{3} x \epsilon^{\mu \nu \rho} g \partial_{\mu} g^{-1} g \partial_{\nu} g^{-1} g \partial_{\rho} g^{-1}
$$

donde $\varphi$ es una coordenada tangencial que parametriza el borde de $\mathcal{M}_{2}$. 


\section{La relación con la gravitación en $2+1$ dimensiones}

La teoría de gravitación de Einstein en espacio tiempo tridimensional [37]-[41] no posee grados de libertad que se propaguen. Esto es análogo a lo que sucede con la teoría de ChernSimons, por lo que ya desde el principio se podría suponer alguna relación entre las dos.

La forma explícita de esta relación, que fue aclarada por primera vez en [35] para el caso de la supergravedad y estudiada en profundidad en [36], se puede expresar de la siguiente manera: formulando la teoría de la gravitación en el formalismo de tríadas, existe un cambio de variables que la mapea en una teoría gauge con acción de Chern-Simons. El grupo de gauge adecuado depende de la signatura del espacio tiempo y del signo de la constante cosmológica.

En el formalismo de tríadas, las variables dinámicas de la gravitación son la tríada o dreibein $e^{a}{ }_{\mu}$ y la conexión de Ricci o conexión de spin $w^{a b}{ }_{\mu}$, las cuales se relacionan con la métrica y la conexión afín de acuerdo con $g_{\mu \nu}=\eta_{a b} e^{a}{ }_{\mu} e^{b}{ }_{\nu} \mathrm{y} \Gamma^{\mu}{ }_{\nu \rho}=\eta_{b c} e_{a}{ }^{\mu} w^{a b}{ }_{\rho} e^{c}{ }_{\nu}+e_{a}{ }^{\mu} \partial_{\rho} e^{a}{ }_{\nu}$, donde $\eta_{a b}=\operatorname{diag}(1,-1,-1)$ es la métrica plana de Minkowski y hemos definido el dreibein inverso $e_{a}^{\mu}$ de modo que $e_{a}{ }^{\mu} e^{b}{ }_{\mu}=\delta_{b}^{a}$. Estas relaciones permiten interpretar la tríada $e^{a}{ }_{\mu}$ como la matriz de transformación que en cada punto lleva del sistema de coordenadas $x^{\mu}$ al sistema localmente minkowskiano.

En términos de estas nuevas variables, la acción de Einstein-Hilbert tridimensional se escribe como

$$
\begin{aligned}
S_{E H}\left[e_{\mu}^{a}, w^{a b}{ }_{\mu}\right] & =\int d^{3} x \sqrt{-g}\left(R_{\mu \nu}[\Gamma] g^{\mu \nu}-\frac{2 \epsilon}{l^{2}}\right)= \\
& =\int d^{3} x \epsilon_{a b c} \epsilon^{\mu \nu \rho}\left(R^{a b}{ }_{\mu \nu} e_{\rho}^{c}+\frac{\epsilon}{3 l^{2}} e_{\mu}^{a}{ }_{\mu} e_{\nu}^{b} e_{\rho}^{c}\right),
\end{aligned}
$$

aquí $R^{a b}{ }_{\mu \nu}=\partial_{\mu} w^{a b}{ }_{\nu}-\partial_{\nu} w^{a b}{ }_{\mu}+w^{a}{ }_{c \mu} w^{c b}{ }_{\nu}-w^{a}{ }_{c \nu} w^{c b}{ }_{\mu}$, y hemos escrito la constante cosmológica como $\epsilon / l^{2} \operatorname{con} \epsilon=0, \pm 1$.

Para relacionar la acción de Einstein con la teoría de Chern-Simons, definimos un campo de gauge $A_{\mu}$ según

$$
A_{\mu}=e^{a}{ }_{\mu} \mathcal{P}_{a}+w^{a}{ }_{\mu} \mathcal{J}_{a}
$$

donde hemos definido $w^{a}{ }_{\mu}=-(1 / 2) \epsilon_{b c}^{a} w^{b c}{ }_{\mu}$ y donde $\mathcal{J}_{a}$ y $\mathcal{P}_{a}$ son generadores de algún grupo de gauge a determinar. La acción de Chern-Simons (5.1) para esta conexión de gauge es idéntica a la forma (5.12) de la acción de Einstein-Hilbert, cuando se imponen sobre los generadores $\mathcal{J}_{a}$ y $\mathcal{P}_{a}$ las reglas de conmutación

$$
\left[\mathcal{J}_{a}, \mathcal{J}_{b}\right]=\epsilon_{a b}{ }^{c} \mathcal{J}_{c}, \quad\left[\mathcal{P}_{a}, \mathcal{P}_{b}\right]=-\frac{\epsilon}{l^{2}} \epsilon_{a b}{ }^{c} \mathcal{J}_{c}, \quad\left[\mathcal{J}_{a}, \mathcal{P}_{b}\right]=\epsilon_{a b}{ }^{c} \mathcal{P}_{c}
$$

y la normalización

$$
\operatorname{Tr}\left(\mathcal{P}_{a} \mathcal{P}_{b}\right)=\operatorname{Tr}\left(\mathcal{J}_{a} \mathcal{J}_{b}\right)=0, \quad \operatorname{Tr}\left(\mathcal{J}_{a} \mathcal{P}_{b}\right)=\eta_{a b}
$$


Por lo tanto, la teoría de la gravitación en $2+1$ dimensiones es equivalente a una teoría de gauge con acción de Chern-Simons y con grupo de gauge generado por las matrices $\mathcal{P}_{a}$ y $\mathcal{J}_{a}$.

De acuerdo con lo que habíamos adelantado, se puede ver que las reglas de conmutación (5.14) dependen del signo de la constante cosmológica, y por lo tanto lo mismo sucede con el grupo de gauge de la teoría de Chern-Simons adecuada para la gravitación.

En el caso de constante cosmológica nula $\epsilon=0$, las reglas de conmutación corresponden a las del grupo $I S O(2,1)$, es decir que la teoría de gravedad es equivalente a una teoría de Chern-Simons cuyo grupo de gauge es el grupo de Poincaré en $2+1$ dimensiones.

En el caso de constante cosmológica negativa $\epsilon=-1$, podemos hacer un cambio de base $\left\{\mathcal{P}_{a}, \mathcal{J}_{a}\right\} \rightarrow\left\{J_{a}^{+}, J_{a}^{-}\right\}$, con $J_{a}^{ \pm}=(1 / 2)\left(\mathcal{J}_{a} \pm l \mathcal{P}_{a}\right)$. Entonces el álgebra de lie del grupo es generada con combinaciones lineales con coeficientes reales de seis matrices $J_{a}^{ \pm}$. Estas matrices cumplen las reglas de conmutación

$$
\left[J_{a}^{+}, J_{b}^{+}\right]=\epsilon_{a b}^{c} J_{c}^{+}, \quad\left[J_{a}^{-}, J_{b}^{-}\right]=\epsilon_{a b}^{c} J_{c}^{-}, \quad\left[J_{a}^{+}, J_{b}^{-}\right]=0
$$

que corresponden a dos copias del álgebra de $S O(2,1)$. Por lo tanto el grupo de gauge adecuado para este caso es localmente isomorfo a $S O(2,1) \times S O(2,1)$, y la acción se desacopla en dos términos de Chern-Simons independientes para las dos conexiones $S O(2,1)$.

En el caso restante de constante cosmológica positiva $\epsilon=1$, nótese que los generadores se pueden representar como $\mathcal{J}_{a}=J_{a}, \mathcal{P}_{a}=(i / l) J_{a}$, donde las matrices $J_{a}$ cumplen reglas de conmutación análogas a las de $\mathcal{J}_{a}$. Por lo tanto podemos decir que el álgebra de Lie es generada con coeficientes reales por el conjunto $\left\{J_{a},(i / l) J_{a}\right\}$, o equivalentemente, con coeficientes complejos por el conjunto $\left\{J_{a}\right\}$. Los generadores $J_{a}$ se pueden representar utilizando las matrices de Pauli $J_{1}=i \sigma_{1}, J_{2}=-i \sigma_{2}, J_{3}=i \sigma_{3}$, de donde se deduce que el grupo en cuestión es $S L(2, \mathbb{C})$.

Para analizar el caso euclídeo, necesitamos definir la rotación de Wick en este contexto. Dado que la tríada en espacio tiempo normal se interpreta como la matriz de transformación que lleva al sistema localmente minkowskiano, la definición adecuada para el espacio euclídeo es como la matriz de transformación que lleva al sistema localmente euclídeo. La relación minkowskiana $g_{\mu \nu}=e^{a}{ }_{\mu} e^{b}{ }_{\nu} \eta_{a b}$ se transforma en la correspondiente relación euclídea $g_{\mu \nu}=$ $-e^{a}{ }_{\mu} e_{\nu}^{b} \delta_{a b}$ si hacemos la substitución $e^{0}{ }_{\mu} \rightarrow i e^{0}{ }_{\mu}$. En cuanto a la conexión de spin, notemos que la relación minkowskiana $\Gamma_{\nu \rho}^{\mu}=\eta_{b c} e_{a}^{\mu} w^{a b}{ }_{\rho} e^{c}{ }_{\nu}+e_{a}{ }^{\mu} \partial_{\rho} e^{a}{ }_{\nu}$, se transforma en la relación euclídea $\Gamma_{\nu \rho}^{\mu}=-\delta_{b c} e_{a}^{\mu} w^{a b}{ }_{\rho} e^{c}{ }_{\nu}+e_{a}{ }^{\mu} \partial_{\rho} e^{a}{ }_{\nu}$, si además cambiamos $w_{\mu}^{1} \rightarrow-i w_{\mu}^{1}, w^{2}{ }_{\mu} \rightarrow-i w^{2}{ }_{\mu}$. Definiremos entonces de esta manera la operación de pasar al espacio euclídeo. Nótese que, a diferencia de la rotación de Wick utilizada en teoría de campos, esta definición no afecta de ninguna forma a la coordenada $x^{0}$.

La rotación de Wick arriba definida genera un factor $i$ que multiplica a la acción de Einstein para los campos euclídeos $e^{a}{ }_{\mu}$ y $W_{\nu}^{a b}$, por lo tanto, la acción equivalente para los campos de gauge será la acción de Chern-Simons, multiplicada por este factor.

Aplicando esta rotación en la conexión (5.13), vemos que podemos absorber los coeficientes $i$ en los generadores, haciendo $\mathcal{P}_{0} \rightarrow i \mathcal{P}_{0}, \mathcal{J}_{1} \rightarrow-i \mathcal{J}_{1}, \mathcal{J}_{2} \rightarrow-i \mathcal{J}_{2}$. De esta manera las 
relaciones de conmutación se transforman en

$$
\left[\mathcal{J}_{a}, \mathcal{J}_{b}\right]=-\epsilon_{a b c} \mathcal{J}_{c}, \quad\left[\mathcal{P}_{a}, \mathcal{P}_{b}\right]=\frac{\epsilon}{l^{2}} \epsilon_{a b c} \mathcal{J}_{c}, \quad\left[\mathcal{J}_{a}, \mathcal{P}_{b}\right]=-\epsilon_{a b c} \mathcal{P}_{c}
$$

Con estos generadores podemos deducir los grupos adecuados al caso euclídeo para cada signo de la constante cosmológica. Haciendo consideraciones análogas a las del caso minkowskiano vemos que: para el caso de constante cosmológica nula el grupo será $I S O(3)$, para constante cosmológica positiva, hacemos el cambio de base $J_{a}^{ \pm}=(1 / 2)\left(\mathcal{J}_{a} \pm l \mathcal{P}_{a}\right)$, con lo cual lo nuevos generadores $J_{a}^{+}$y $J_{a}^{-}$cumplen separadamente reglas de conmutación de $S O(3)$, por lo tanto el grupo adecuado será $S O(3) \times S O(3)$, para el caso con constante cosmológica negativa, el grupo será $S L(2, \mathbb{C})$, generado por $\left\{\mathcal{J}_{a},(i / l) \mathcal{P}_{a}\right\}$.

\subsection{La acción de Chern-Simons no conmutativa}

\section{Definición}

Definiremos la acción de Chern-Simons en espacio no conmutativo en la forma que hemos explicado en la sección (4.3) simplemente subtituyendo en la acción de Chern-Simons usual (5.1) (escrita en forma matricial) los campos $A_{\mu}$ por sus análogos no conmutativos $\hat{A}_{\mu}$ y los productos normales por productos estrella. Con esto obtenemos

$$
S_{C S}^{\theta}\left[A_{\mu}\right]=\frac{\kappa}{4 \pi} \operatorname{Tr} \int_{\mathcal{M}} d^{3} x \epsilon^{\mu \nu \rho}\left(\hat{A}_{\mu} * \partial_{\nu} \hat{A}_{\rho}+\frac{2}{3} \hat{A}_{\mu} * \hat{A}_{\nu} * \hat{A}_{\rho}\right),
$$

fórmula que define la acción de Chern-Simons no conmutativa ${ }^{2}$. Esta acción ha sido estudiada en [66]-[78].

Obsérvese que, como estudiaremos en detalle más adelante, tanto la diferenciabilidad cuanto la invarianza de gauge de esta acción dependen de poder realizar rotaciones cíclicas de los productos estrella involucrados. Tales rotaciones se pueden realizar supuestas las adecuadas condiciones de contorno.

Además, la presencia de la matriz $\theta^{\mu \nu}$ en el producto estrella, rompe la invarianza general de coordenadas de la acción y por lo tanto la acción de Chern-Simons no conmutativa no es una acción topológica, depende de la métrica y del sistema de coordenadas privilegiado donde se define el producto estrella.

Supongamos que el producto estrella está definido en el sistema $\left(x^{0}, x^{1}, x^{2}\right)$. Es siempre posible encontrar una transformación de Lorentz que lleve a un nuevo sistema de coordenadas $\left(x^{0^{\prime}}, x^{1^{\prime}}, x^{2^{\prime}}\right)$, donde la dirección $x^{0^{\prime}}$ es conmutativa, es decir que $\left[x^{0^{\prime}}, x^{1^{\prime}}\right]=\left[x^{0^{\prime}}, x^{2^{\prime}}\right]=0$. Este sistema de coordenadas nos será útil más adelante, al estudiar el comportamiento de la teoría de Chern-Simons no conmutativa en variedades con borde.

\footnotetext{
${ }^{2} \mathrm{~A}$ lo largo de todo esta tesis hemos evitado usar la notación de formas diferenciales $A=A_{\mu} d x^{\mu}$, ya que puede inducir a confusión en el caso no conmutativo, especialmente al usar el teorema de Stokes
} 
Relación con el efecto Hall

Supongamos la variedad espacio temporal es $\mathbb{R}^{3}$ y que los campos de gauge se anulan lo suficientemente rápido en el infinito, de manera de poder realizar sin riesgo rotaciones cíclicas en los productos estrella. En tal caso podemos reescribir (5.18) según

$$
S_{C S}^{\theta}\left[A_{0}, A_{i}\right]=\frac{\kappa}{4 \pi} \operatorname{Tr} \int_{\mathcal{M}} d^{3} x \epsilon^{i j}\left(\hat{A}_{0} * \hat{F}_{i j}-\hat{A}_{i} * \partial_{0} \hat{A}_{j}\right),
$$

El comportamiento que hemos supuesto para los campos en el infinito nos permite omitir los productos estrella bajo el signo integral, ya que el término de borde se anula. Entonces nos queda

$$
S_{C S}^{\theta}\left[A_{0}, A_{i}\right]=\frac{\kappa}{4 \pi} \operatorname{Tr} \int_{\mathcal{M}} d^{3} x \epsilon^{i j}\left(\hat{A}_{0} \hat{F}_{i j}-\hat{A}_{i} \partial_{0} \hat{A}_{j}\right),
$$

lo que coincide con la acción a la que hemos mencionado al describir el efecto Hall en el límite continuo. Por lo tanto la acción efectiva para el estudio del efecto Hall desde un punto de vista fluidodinámico es la acción de Chern-Simons no conmutativa [22]. 


\section{Capítulo 6}

\section{Término de Chern-Simons inducido por integración de fermiones}

En este capítulo describiremos cómo se induce un término de Chern-Simons en espacio no conmutativo por fluctuaciones de campos fermiónicos masivos, exactamente como sucede en el caso usual conmutativo. Nos concentraremos solamente en la parte que viola paridad de la acción efectiva, descartando los términos que la preservan. Los resultados aquí descriptos forman parte de las contribuciones originales de esta tesis.

\subsection{Fermiones acoplados a un campo de gauge en es- pacio no conmutativo}

Llamaremos $\hat{\psi}(x)$ a nuestros campos fermiónicos de spin $1 / 2$ definidos en $2+1$ dimensiones no conmutativas. Como hemos visto en el capítulo 4, cuando el espacio es no conmutativo, existen tres representaciones posibles del la acción del grupo de gauge sobre los campos de materia, aún en el caso $U(1)$, a saber las representaciones fundamental, antifundamental y adjunta, cuya acción se muestra en las ecuaciones (4.2).

De acuerdo a la representación elegida, utilizaremos como acción $S^{\theta}\left[\hat{\psi}, \hat{A}_{\mu} ; m\right]$ para los campos fermiónicos de masa $m$ acoplados a un campo de gauge $\hat{A}_{\mu}$ en espacio no conmutativo, la acción definida en las ecuaciones (4.15) y (4.17) de la sección 4.3.

La acción efectiva está definida de acuerdo con

$$
e^{i \Gamma[\hat{A} ; m]}=Z[\hat{A} ; m]=\int \mathcal{D} \hat{\psi} \mathcal{D} \overline{\hat{\psi}} e^{i S^{\theta}[\hat{\psi}, \hat{A} ; m]}
$$

que es el análogo no conmutativo de la expresión (5.4).

De acuerdo a las consideraciones generales de la sección 2.3, en el desarrollo perturbativo de la acción efectiva, la única modificación de las reglas de Feynman es el factor de fase dependiente de $\theta^{\mu \nu}$ en cada vértice. 

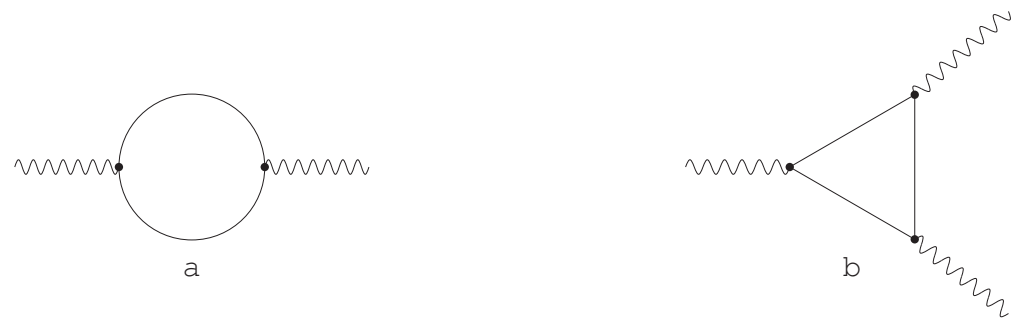

Figura 6.1: Diagramas de polarización de vacío y triángulo,para fermiones en la representación fundamental, que contribuyen a $\Pi^{\mu \nu}(p ; m)$ y a $\Gamma^{\mu \nu \rho}(p, q ; m)$ respectivamente.

\subsection{Representaciones fundamental y antifundamental}

Los cálculos de la acción efectiva para fermiones en la representación fundamental y en la antifundamental conducen al mismo resultado. Describiremos primero el caso de la representación fundamental. Como en el cálculo original [24], nos concentraremos aquí en las contribuciones a $\Gamma(\hat{A} ; m)$ no invariantes de paridad provenientes de los diagramas de polarización de vacío y triángulo ${ }^{1}$, que se ven en la Fig. 6.1, dando origen a los términos

$$
\begin{aligned}
i \Gamma_{\text {impar }}[\hat{A} ; m]=\operatorname{Tr} \int \frac{d^{3} p}{(2 \pi)^{3}}( & \frac{1}{2} \tilde{A}_{\mu}(p) \Pi^{\mu \nu}(p ; m) \tilde{A}_{\nu}(-p)+ \\
& \left.+\frac{1}{3} \int \frac{d^{3} q}{(2 \pi)^{3}} \Gamma^{\mu \nu \rho}(p, q ; m) \tilde{A}_{\mu}(p) \tilde{A}_{\nu}(q) \tilde{A}_{\rho}(-p-q)\right)\left.\right|_{i m p a r}
\end{aligned}
$$

Aquí los tensores $\Pi^{\mu \nu}(p ; m)$ y $\Gamma^{\mu \nu \rho}(p, q ; m)$ se calculan usando las reglas de Feynmann descriptas en el capítulo 2.3, lo que conduce a

$$
\begin{aligned}
\Pi^{\mu \nu}(p ; m) & =\int \frac{d^{3} k}{(2 \pi)^{3}} \operatorname{tr}\left(\gamma^{\mu} \frac{\not k+m}{k^{2}-m^{2}} \gamma^{\nu} \frac{\not k+\not h+m}{(k+p)^{2}-m^{2}}\right), \\
\Gamma^{\mu \nu \rho}(p, q ; m) & =-e^{-\frac{i}{2} p_{\lambda} \theta^{\lambda \delta} q_{\delta}} \int \frac{d^{3} k}{(2 \pi)^{3}} \operatorname{tr}\left(\gamma^{\mu} \frac{(\not k+m)}{k^{2}-m^{2}} \gamma^{\nu} \frac{(\not k-\not h+m)}{(k-q)^{2}-m^{2}} \gamma^{\rho} \frac{(\not k+\not p+m)}{(k+p)^{2}-m^{2}}\right) .
\end{aligned}
$$

Como se puede ver, estos diagramas no contienen parte no plana, de donde vemos que, cuando los fermiones se encuentran en la representación fundamental, no habrá contribución no plana a la parte impar de la acción efectiva (esto coincide con lo que se observa en [66] para fermiones sin masa). Por lo tanto, tanto en el término cuadrático como en el cúbico, no hay factores de fase que contengan a las variables de integración, por los que podemos asegurar que en el caso de la representación fundamental no se presentará el fenómeno de mezcla infrarrojo-ultravioleta. La única modificación debida a la no conmutatividad es el factor de fase dependiente de $\theta^{\mu \nu}$ en $\Gamma^{\mu \nu \rho}(p, q ; m)$, asociado a las patas externas en el término cúbico, el cual no es otra cosa que el producto estrella escrito en el espacio de impulsos. Esto

\footnotetext{
${ }^{1}$ La presencia de correcciones provenientes de otros diagramas será analizada en la sección 6.4.
} 
nos permite anticipar que el resultado para $\Gamma_{\text {impar }}[\hat{A} ; m]$ será análogo al caso conmutativo, excepto que el producto estrella reemplazará al producto ordinario.

La regularización de las integrales divergentes (6.3) y (6.4) se puede realizar mediante el método de Pauli-Villars, introduciendo en la acción original (4.15) nuevos campos espinoriales masivos y con estadística bosónica. La masa $M$ de estos campos reguladores se hará tender a infinito al final de los cálculos. Estos campos dan origen a diagramas adicionales, idénticos a los anteriores, excepto que la masa del regulador $M$ aparece en lugar de la masa física $m$. Por lo tanto, en la fórmula (6.2) para $\Gamma_{i m p a r}(\hat{A} ; m)$, en lugar de los tensores (6.3) y (6.4) aparecen los tensores regularizados

$$
\begin{aligned}
\left.\Pi^{\mu \nu}(p ; m)\right|^{r e g} & =\lim _{M \rightarrow \infty}\left(\Pi^{\mu \nu}(p ; m)+\Pi^{\mu \nu}(p ; M)\right), \\
\left.\Gamma^{\mu \nu \rho}(p, q ; m)\right|^{r e g} & =\lim _{M \rightarrow \infty}\left(\Gamma^{\mu \nu \rho}(p, q ; m)+\Gamma^{\mu \nu \rho}(p, q ; M)\right) .
\end{aligned}
$$

Ahora bien, estamos interesados solamente en la parte impar de la acción efectiva. En la expresión (6.2), el campo de gauge $\hat{A}_{\mu}$ transforma como vector frente a transformaciones de paridad, $\hat{A}_{\mu} \rightarrow-\hat{A}_{\mu}$ y sus índices de Lorentz están contraídos con los índices de los tensores (6.5) y (6.6). Por lo tanto la parte impar de la acción efectiva provendrá de la parte pseudotensorial de (6.5) y (6.6). Para aislar esta parte, en primer lugar expandiremos los productos del numerador en cada uno de los integrandos en (6.3) y (6.4) (y en las integrales análogas correspondientes al campo regulador) y efectuaremos las trazas sobre las matrices de Dirac $\gamma^{\mu}$. Mantendremos sólo los términos con un número impar de matrices de Dirac, ya que son éstos los que se comportan como pseudotensores. Entonces

$$
\begin{gathered}
\left.\Pi^{\mu \nu}(p ; m)\right|_{\text {impar }}=2 i m \epsilon^{\mu \rho \nu} p_{\rho} \int \frac{d^{3} k}{(2 \pi)^{3}} \frac{1}{\left(k^{2}-m^{2}\right)\left((k+p)^{2}-m^{2}\right)}, \\
\left.\Gamma^{\mu \nu \rho}(p, q ; m)\right|_{\text {impar }}=2 i m e^{-\frac{i}{2} p_{\lambda} \theta^{\lambda \delta} q_{\delta}} \int \frac{d^{3} k}{(2 \pi)^{3}} \times \\
\times\left(\frac{\epsilon^{\mu \rho \nu}\left(k^{2}-m^{2}\right)+2\left(\epsilon^{\mu \rho \alpha} k^{\nu} p_{\alpha}+\epsilon^{\nu \rho \alpha} k^{\mu} q_{\alpha}\right)+\epsilon^{\mu \nu \alpha}\left(q_{\alpha} p^{\rho}+p_{\alpha} q^{\rho}\right)+\left(\epsilon^{\mu \rho \nu} \eta^{\alpha \beta}-\eta^{\mu \nu} \epsilon^{\rho \alpha \beta}\right) q_{\alpha} p_{\beta}}{\left(k^{2}-m^{2}\right)\left((k-q)^{2}-m^{2}\right)\left((k+p)^{2}-m^{2}\right)}\right),
\end{gathered}
$$

y expresiones análogas para la parte proveniente del campo regulador (los segundos términos de (6.5) y (6.6)). A continuación, haremos en ambas integrales (6.7) y (6.8) el cambio de variables $k_{\mu} \rightarrow|m| s_{\mu}$ (en las integrales similares correspondientes al campo regulador, el cambio será $k_{\mu} \rightarrow|M| s_{\mu}$ ), y desarrollaremos el integrando a primer orden en $p /|m|$ y $q /|m|$ (y respectivamente en $p /|M|$ y $q /|M|$ ). Este desarrollo corresponde a tomar límite de baja energía de la acción efectiva, es decir $p_{0} \ll|m|$ (en el caso del campo regulador $p_{0} \ll|M|$ no conlleva ninguna aproximación, ya que por definición $M$ es mayor que cualquier valor finito). Con esto llegamos a la expresión simplificada

$$
\left.\Pi^{\mu \nu}(p ; m)\right|_{\text {impar }}=2 i \frac{m}{|m|} \epsilon^{\mu \rho \nu} p_{\rho} \int \frac{d^{3} s}{(2 \pi)^{3}} \frac{1}{\left(s^{2}-1\right)^{2}}=
$$




$$
\begin{aligned}
& =\frac{m}{|m|} \frac{1}{4 \pi} \epsilon^{\mu \rho \nu} i p_{\rho}, \\
\left.\Gamma^{\mu \nu \rho}(p, q ; m)\right|_{\text {impar }} & =2 i \frac{m}{|m|} \epsilon^{\mu \rho \nu} e^{-\frac{i}{2} p_{\lambda} \theta^{\lambda \delta} q_{\delta}} \int \frac{d^{3} s}{(2 \pi)^{3}} \frac{1}{\left(s^{2}-1\right)^{2}}= \\
& =i \frac{m}{|m|} \frac{1}{4 \pi} \epsilon^{\mu \rho \nu} e^{-\frac{i}{2} p_{\lambda} \theta^{\lambda \delta} q_{\delta}},
\end{aligned}
$$

donde en la segunda línea de cada ecuación hemos efectuado la integral en la variable $s_{\mu}$. A estas expresiones se deben sumar los resultados provenientes de los diagramas del campo regulador $^{2}$ obteniéndose como resultado final

$$
\begin{aligned}
\left.\Pi_{f}^{\mu \nu}(p ; m)\right|_{\text {impar }} ^{r e g} & =\left(\frac{m}{|m|}+\frac{M}{|M|}\right) \frac{1}{4 \pi} \epsilon^{\mu \rho \nu} i p_{\rho}, \\
\left.\Gamma_{f}^{\mu \nu \rho}(p, q ; m)\right|_{\text {impar }} ^{r e g} & =i\left(\frac{m}{|m|}+\frac{M}{|M|}\right) \frac{1}{4 \pi} \epsilon^{\mu \rho \nu} e^{-\frac{i}{2} p_{\lambda} \theta^{\lambda \delta} q_{\delta}} .
\end{aligned}
$$

Reemplazando estas expresiones en (6.2), obtenemos para la parte impar de la acción efectiva, la acción de Chern-Simons escrita en espacio de impulsos, con lo que arribamos a nuestro principal resultado de esta sección: el orden dominante en $\partial / m$ de la parte que viola paridad de la acción efectiva invariante de gauge está, para la representación fundamental, dado por la acción de Chern-Simons

$$
\begin{aligned}
\Gamma_{\text {impar }}^{f}[\hat{A} ; m] & =\frac{1}{2}\left(\frac{m}{|m|}+\frac{M}{|M|}\right) S_{C S}^{\theta}(\hat{A})+O\left(\partial^{2} / m^{2}\right)= \\
& = \pm S^{\theta}{ }_{C S}(\hat{A})+O\left(\partial^{2} / m^{2}\right) .
\end{aligned}
$$

Como es evidente, el signo relativo de las contribuciones fermiónicas con respecto a las de los campos reguladores, depende de la elección del signo de la masa de estos últimos (por supuesto, la parte divergente de estos diagramas se cancela independientemente de esta elección). En la primera línea de (6.13) hemos elegido estos signos de manera que los dos términos se sumen para dar el resultado de Chern-Simons en la segunda línea (dado que se trata de fermiones masivos, si hubiéramos elegido signos diferentes de ninguna manera significa que la acción resultante conserva paridad, la violación de paridad reaparecería en potencias mayores de $\left.p_{\mu} /|m|[28],[29]^{3}\right)$.

Como se podía esperar, la acción efectiva es invariante de gauge aún bajo transformaciones de gauge grandes. Esto se debe a que hemos tomado en cuenta ambas fuentes de violación de paridad: aquella originada en el término de masa de los fermiones, que no es invariante

\footnotetext{
${ }^{2} \mathrm{Si}$ bien las integrales involucradas en la parte impar de la acción efectiva son convergentes, lo que podría hacer pensar pensar al lector distraído que la regularización no es necesaria, el diagrama completo es divergente, y una renormalización consistente de la teoría exige la regulación de todo el diagrama.

${ }^{3}$ Ver la sección 6.4
} 

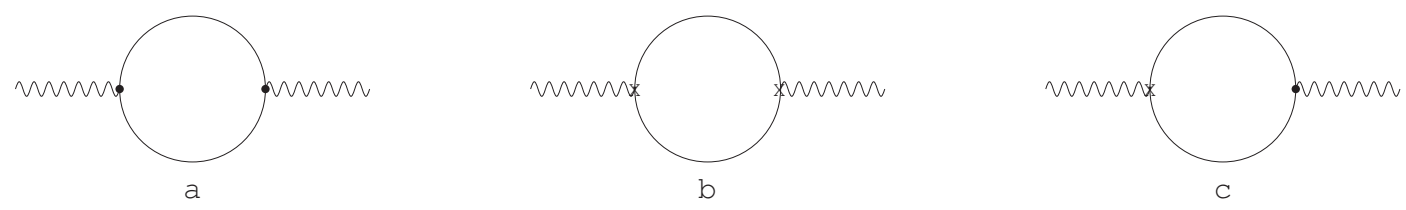

Figura 6.2: Diagramas que contribuyen a $\Pi^{\mu \nu}(p ; m)$ para fermiones en la representación adjunta. El vértice punteado coincide con el acoplamiento de $\hat{A}_{\mu}$ con fermiones en la representación fundamental, mientras que el vértice con una cruz coincide con el correspondiente a fermiones en la anti-fundamental.

de paridad, y la relacionada con la prescripción de regularización, la cual requiere la introducción de una masa $M$. En el caso de fermiones no masivos, la contribución proveniente de la masa física no estaría presente ${ }^{4}$.

Se debe resaltar aquí que en el presente cálculo, el límite del regulador conmuta con el limite conmutativo. En efecto, si tomamos $\theta^{\mu \nu} \rightarrow 0$ antes de $M \rightarrow \infty$, el resultado corresponde al de la integración de fermiones en espacio conmutativo, es decir, a la acción de Chern-Simons conmutativa, la cual obviamente coincide con lo que se obtiene si se toma el límite conmutativo en el resultado ya regulado (6.13).

Como se ha dicho antes, el cálculo de la parte no invariante de paridad de la acción efectiva para fermiones en la representación antifundamental conduce al mismo resultado. Hay un cambio de signo en la constante de acoplamiento en cada vértice, que es compensado por el diferente ordenamiento de los campos en la derivada covariante.

\subsection{Representación Adjunta}

En el caso de la representación adjunta, los diagramas que contribuyen a $\Pi^{\mu \nu}(p ; m)$ se muestran en la figura 6.2. Como se puede ver, los diagramas $6.2 \mathrm{a}$ y $6.2 \mathrm{~b}$ coinciden con los que se obtienen en la representación fundamental y anti-fundamental respectivamente, por lo que no tendrán parte no plana y contribuirán a la parte impar de la acción efectiva, cada uno, con el resultado calculado previamente (6.13). En cuanto al diagrama 6.2c, la contribución resultante será no plana, como se ve en la expresión

$$
\Pi_{c}^{\mu \nu}(p ; m)=-\int \frac{d^{3} k}{(2 \pi)^{3}} e^{-i p_{\lambda} \theta^{\lambda \delta} k_{\delta}} \operatorname{tr}\left(\gamma^{\mu} \frac{\not k-\not p+m}{(k-p)^{2}-m^{2}} \gamma^{\nu} \frac{\not k+m}{k^{2}-m^{2}}\right) .
$$

Como en la sección previa, debemos separar la parte impar (pseudo-tensorial) de la expresión anterior. Para esto, nuevamente mantendremos los términos que contienen un número impar

\footnotetext{
${ }^{4}$ La invarianza de gauge de la acción efectiva bajo transformaciones grandes en este caso será discutida en la sección 6.4
} 
de matrices de Dirac, es decir

$$
\left.\Pi_{c}^{\mu \nu}(p ; m)\right|_{\text {impar }}=-2 i m \epsilon^{\mu \nu \rho} p_{\rho} \int \frac{d^{3} k}{(2 \pi)^{3}} \frac{e^{-i p_{\lambda} \theta^{\lambda \delta} k_{\delta}}}{\left(k^{2}-m^{2}\right)\left((k-p)^{2}-m^{2}\right)},
$$

como antes, a esto se debe sumar una expresión análoga proveniente del campo regulador. $\mathrm{Al}$ efectuar ahora el cambio de variables $k_{\mu}=|m| s_{\mu}$ (y el correspondiente $k_{\mu}=|M| s_{\mu}$ para el campo de Pauli-Villars), la expresión anterior se transforma en

$$
\left.\Pi_{c}^{\mu \nu}(p ; m)\right|_{i m p a r}=-2 \frac{m}{|m|} \epsilon^{\mu \nu \rho} i p_{\rho} \int \frac{d^{3} s}{(2 \pi)^{3}} \frac{e^{-i|m| p_{\lambda} \theta^{\lambda \delta} s_{\delta}}}{\left(s^{2}-1\right)\left(\left(s-\frac{p}{|m|}\right)^{2}-1\right)} .
$$

Nótese que luego del cambio de variables, en (6.16) aparecen dos variables adimensionales que contienen el impulso externo $p_{\mu}$, a saber $p_{\mu} /|m|$ y $|m| p_{\mu} \theta^{\mu \nu}$. Expandiendo primero (6.16) a primer orden en $p_{\mu} /|m|$, es decir considerando energías mucho menores que la masa $p_{0} \ll|m|$, obtenemos

$$
\left.\Pi_{c}^{\mu \nu}(p ; m)\right|_{\text {impar }} ^{r e g}=-2 i \epsilon^{\mu \nu \rho} p_{\rho}\left(\frac{m}{|m|} \int \frac{d^{3} q}{(2 \pi)^{3}} \frac{e^{-i|m| p_{\lambda} \theta^{\lambda \delta} q_{\delta}}}{\left(q^{2}-1\right)^{2}}-\lim _{M \rightarrow \infty} \frac{M}{|M|} \int \frac{d^{3} q}{(2 \pi)^{3}} \frac{e^{-i|M| p_{\lambda} \theta^{\lambda \delta} q_{\delta}}}{\left(q^{2}-1\right)^{2}}\right),
$$

aquí, la contribución del regulador ha sido explícitamente escrita. Si la energía es lo suficientemente pequeña $p_{0} \ll 1 /(|m \theta|)$, podemos expandir también el primer término de (6.17) en potencias de la segunda variable adimensional $|m| p_{\mu} \theta^{\mu \nu}$. En cuanto al segundo término, se debe hacer notar que, dado que al final de los cálculos debemos tomar el límite del regulador $M \rightarrow \infty$, no es posible hacer la misma expansión. Por lo tanto tenemos el resultado

$$
\begin{aligned}
\left.\Pi_{c}^{\mu \nu}(p ; m)\right|_{\text {impar }} ^{r e g} & =-2 i \epsilon^{\mu \nu \rho} p_{\rho}\left(\frac{m}{|m|} \int \frac{d^{3} q}{(2 \pi)^{3}} \frac{1}{\left(q^{2}-1\right)^{2}}-\lim _{M \rightarrow \infty} \frac{M}{|M|} \int \frac{d^{3} q}{(2 \pi)^{3}} \frac{e^{-i|M| p_{\lambda} \theta^{\lambda \delta} q_{\delta}}}{\left(q^{2}-1\right)^{2}}\right)= \\
& =-i \frac{m}{|m|} \frac{1}{4 \pi} \epsilon^{\mu \nu \rho} i p_{\rho},
\end{aligned}
$$

en la segunda línea se ha calculado explícitamente la integral del primer término. La integral del segundo término se anula debido al factor oscilatorio en el integrando. Entonces no hay contribución del regulador en el diagrama no plano 6.2c.

Por lo tanto la parte pseudotensorial de $\Pi^{\mu \nu}(p ; m)$ en la representación adjunta está dada por

$$
\begin{aligned}
\left.\Pi_{a d j}^{\mu \nu}(p ; m)\right|_{i m p a r} ^{r e g} & =\left.\Pi_{a}^{\mu \nu}(p ; m)\right|_{i m p a r} ^{r e g}+\left.\Pi_{b}^{\mu \nu}(p ; m)\right|_{i m p a r} ^{r e g}+\left.2 \Pi_{c}^{\mu \nu}(p ; m)\right|_{i m p a r} ^{r e g}= \\
& =\frac{1}{2 \pi} \epsilon_{\mu \nu \rho} p_{\rho} \frac{M}{|M|} .
\end{aligned}
$$



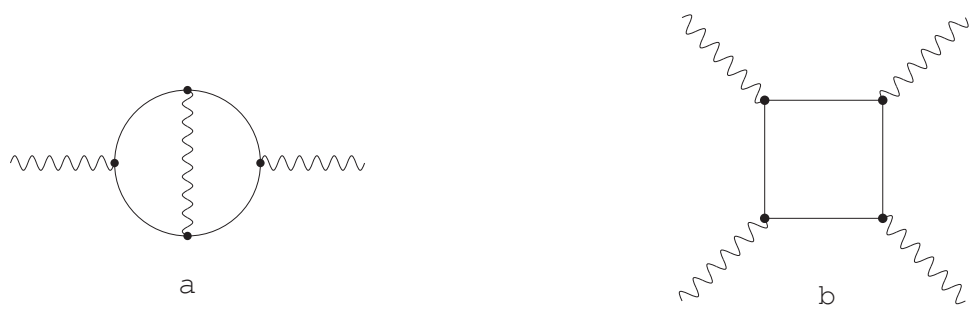

Figura 6.3: Diagramas que no hemos considerado en nuestros cálculos. Los diagramas con loops superiores del tipo a son excluidos en el caso conmutativo por el teorema de Coleman y Hill, y no deberían contribuir aún en el caso no conmutativo si la acción efectiva debe ser invariante bajo transformaciones de gauge largas. Diagramas del tipo b contribuirán a la acción efectiva con potencias superiores de $\hat{A}_{\mu}$.

Nótese que las contribuciones en la representación adjunta a $\left.\Pi^{\mu \nu}(p, m)\right|_{i m p a r} ^{r e g}$ provenientes de los campos físicos se cancelan y la única contribución no nula proviene en su totalidad de los campos reguladores.

Esto da cuenta de la parte cuadrática de la acción efectiva. En cuanto al término cúbico, puede computarse explícitamente o ajustarse de manera de tener un resultado invariante de gauge. En cualquier caso, el resultado para la parte que viola paridad de la acción efectiva para fermiones en la representación adjunta está dado, al orden dominante en $\partial$, por la acción de Chern-Simons

$$
\Gamma_{\text {impar }}^{a d}[\hat{A} ; m]= \pm \hat{S}_{C S}(\hat{A})+O\left(\partial^{2}\right) .
$$

Como antes, el resultado es invariante de gauge aún bajo transformaciones grandes.

Se debe resaltar que (6.20) da una acción efectiva no trivial aún en el límite $\theta^{\mu \nu} \rightarrow 0$, en el cual, para el caso abeliano, los fermiones en la adjunta se desacoplan del campo de gauge. Como se ha observado en otros casos [79]-[84], esto se debe al hecho de que este límite no conmuta con el limite del regulador $M \rightarrow \infty$, y tiene su origen en la mezcla infrarrojo-ultravioleta IR/UV, característica de los modelos no conmutativos.

\subsection{Alcances y limitaciones del cálculo}

Llegados a este punto, se hace necesario plantearse cual es la generalidad de los cálculos realizados en las secciones precedentes y de que manera se podrían mejorar. Sobresalen los siguientes puntos

- Más loops: hemos realizado un cálculo a un loop, lo que inmediatamente plantea la posibilidad de generalizarlo considerando la contribución de gráficos con dos o mas loops, como en la Fig 6.3a. Nótese que estos gráficos contienen propagadores del campo de gauge $\hat{A}_{\mu}$, lo que implica que al incluirlos estamos integrando también sobre fluctuaciones de este campo, y no solamente de los fermiones, como hemos hecho hasta ahora. 
A este respecto, para el caso abeliano conmutativo, existe un teorema general debido a Coleman y Hill [30], que establece que el coeficiente del término Chern-Simons en la parte impar de la acción efectiva no recibe correcciones provenientes de loops superiores en teoría de perturbaciones, es decir, que no se renormaliza, (este teorema incluye la posibilidad de campos escalares y fermiones masivos en la acción).

Para el caso no abeliano, existe abundante evidencia de que el teorema también se cumple. En particular, dado que en ese caso el coeficiente del termino de Chern-Simons debe estar cuantizado, la invarianza de gauge de la teoría efectiva frente a transformaciones grandes exige que este coeficiente no se renormalice. Sin embargo, la versión no abeliana del teorema de Coleman-Hill fue demostrada sólo recientemente usando el método de holomorficidad de Seiberg [31], y por métodos mas directos en [32]. Estos resultados han sido extendidos en parte al presente caso no conmutativo en [77].

- Potencias superiores de $\hat{A}_{\mu}$ : nos hemos limitado a las contribuciones a la parte impar de la acción efectiva que provienen de los diagramas de un loop con dos y tres patas externas. Es natural preguntarse acerca de los diagramas de un loop, pero con más patas externas, el tipo de la figura 6.3b. Estos gráficos corresponden a términos en $\Gamma_{\text {impar }}(\hat{A} ; m)$ con potencias del campo de gauge mayores que tres, y por lo tanto no afectan a la parte de Chern-Simons ya calculada.

En el caso no masivo, la violación de paridad proviene de la masa $M$ del campo regulador. Por lo tanto, los gráficos con más patas externas, que son convergentes y no reciben correcciones provenientes del regulador, serán pares y no afectaran a $\Gamma_{\text {impar }}(\hat{A} ; m)$.

En el caso masivo, los gráficos con más patas externas, si bien continúan siendo convergentes y por lo tanto no reciben contribuciones que contengan la masa del regulador, contienen potencias de la masa física $m$. Por lo tanto no está en principio vedado que contribuyan a la violación de paridad. (En el punto siguiente se discute una posible forma de la acción efectiva que contiene contribuciones de estos gráficos)

- Ordenes superiores en $p /|m|$ : finalmente, nuestro cálculo fue hecho solo al primer orden en la variable adimensional $p /|m|$. El siguiente orden en esta expansión corresponde en el caso usual conmutativo abeliano a la extensión de Chern-Simons en derivadas mas altas estudiada en [28],[29]. En el caso no conmutativo se obtiene una fórmula análoga, donde los productos ordinarios han sido reemplazados por productos estrella.

$$
S_{H C S}^{\prime}=\frac{1}{|m|} \int d^{3} x \epsilon^{\mu \nu \rho} \hat{F}_{\mu}^{\prime} * \partial_{\nu} \hat{F}_{\rho}^{\prime}
$$

aquí $\hat{F}_{\mu}^{\prime}=\epsilon_{\mu}^{\nu \rho} \partial_{\mu} \hat{A}_{\rho}$. Como se puede ver, esta fórmula no es invariante de gauge. La forma de una extensión en derivadas mas altas que sea invariante de gauge, deberá necesariamente estar formulada en términos de la derivada covariante, y además 
deberá contener la curvatura completa, es decir $\hat{F}_{\mu}=\epsilon_{\mu}^{\nu \rho}\left(\partial_{\nu} \hat{A}_{\rho}+\hat{A}_{\mu} * \hat{A}_{\rho}\right)$. Una forma razonable no conmutativa de la extensión de Chern-Simons en derivadas mas altas es

$$
S_{H C S}^{\theta}=\frac{1}{|m|} \int d^{3} x \epsilon^{\mu \nu \rho} \hat{F}_{\mu} * \hat{D}_{\nu} \hat{F}_{\rho}
$$

Nótese que debido a la presencia de los términos cuadráticos en $\hat{A}_{\mu}$ y en $\hat{F}_{\mu}$, la acción (6.22) contiene potencias superiores de $\hat{A}_{\mu}$, por lo tanto provendrá de gráficos con más patas externas, del tipo discutidos en el punto anterior.

Sería una continuación interesante de este trabajo, verificar que la forma invariante de gauge (6.22) se obtiene al calcular explícitamente las contribuciones de los gráficos adecuados.

- El caso no masivo: Mencionemos aquí que la parte impar de la acción efectiva para fermiones no masivos en $2+1$ dimensiones ha sido calculada en [66], donde también ha sido analizada su relación con el modelo de Wess-Zumino-Witten.

\subsection{Conclusiones}

Resumiremos aquí los principales resultados de éste capítulo

- Hemos calculado la acción efectiva para fermiones en espacio no conmutativo, en las diferentes representaciones, mostrando que cuando se toman en cuenta las contribuciones del regulador se obtiene un resultado invariante de gauge, aún bajo transformaciones de gauge grandes. En todos los casos la forma de la acción efectiva impar invariante de gauge es una acción de Chern-Simons no conmutativa.

- Para las representaciones fundamental y anti-fundamental, el cálculo es completamente análogo al caso conmutativo, ya que las únicas contribuciones provienen de diagramas planos, en los cuales el factor de fase sale fuera de las integrales.

- Para la representación adjunta, en la cual intervienen diagramas no planos, el resultado invariante de gauge no trivial (6.20) es completamente originado por los campos reguladores, lo que implica que el límite conmutativo $\theta^{\mu \nu} \rightarrow 0$ no conmuta con el límite de la regularización $M \rightarrow \infty$. Este es el fenómeno de mezcla infrarrojo-ultravioleta por lo que nuestro cálculo es el análogo tridimensional del cálculo efectuado en [79]. 


\title{
Capítulo 7
}

\section{La acción de Chern-Simons no conmutativa en variedades con borde}

\begin{abstract}
Aquí estudiaremos algunos aspectos de la acción de Chern-Simons en variedades compactas. En particular, describiremos las condiciones de contorno necesarias para hacer que la acción de Chern-Simons definida en una variedad con borde sea diferenciable e invariante de gauge. Además estudiaremos las transformaciones de simetría de la acción que preservan estas condiciones de contorno. Finalmente estableceremos la conexión entre la acción de Chern-Simons no conmutativa y la acción quiral de WessZumino-Witten. Lo que aquí se expone constituye otro de los resultados originales de la tesis
\end{abstract}

\subsection{El efecto del borde y la derivada de la acción}

Cuando se considera la teoría de Chern-Simons no conmutativa en una variedad con borde, emergen complicaciones debido a la presencia del producto de Moyal. En esta sección seguiremos los pasos delineados en [39] para tratar la acción de Chern-Simons en una variedad con borde, adecuadamente adaptados al presente caso no conmutativo. Tales pasos son:

1. En primer lugar estableceremos las condiciones de contorno sobre el campo de gauge $\hat{A}_{\mu}$ de manera de asegurar la diferenciabilidad de la acción.

2. En segundo lugar, estableceremos las simetrías del problema, encontrando las transformaciones $\hat{g}: \mathcal{M} \rightarrow G$ que preserven esas condiciones de contorno.

3. El último paso en este procedimiento, consiste en definir el grupo de transformaciones de gauge como aquéllas $\hat{g}$ que son generadas por el vínculo ${ }^{1}$. Dado que el desarrollo de

\footnotetext{
${ }^{1}$ Estas serán las simetrías a ser "gaugeadas", es decir eliminadas del espectro de la teoría mediante una proyección adecuada. El resto de las simetrías serán simetrías observables del problema.
} 
este último paso requiere de la formulación hamiltoniana de la teoría, que casi siempre es problemática en el caso no conmutativo, no desarrollaremos esta parte.

\section{Condiciones de contorno}

Veamos primero cuales son las condiciones de contorno adecuadas para que la acción sea diferenciable, es decir que exista una derivada variacional. Al variar la acción para encontrar las ecuaciones de movimiento, es necesario manipular los productos estrella para obtener una expresión cerrada para la derivada variacional. Estas manipulaciones dan origen a términos de borde, que pondrían en duda en principio la diferenciabilidad de la acción. Explícitamente

$$
\begin{aligned}
\delta S_{C S}^{\theta}[\hat{A}]= & \frac{\kappa}{4 \pi} \operatorname{Tr} \int_{\mathcal{M}} d^{3} x \epsilon^{\mu \nu \rho}\left(\delta \hat{A}_{\mu} * \partial_{\nu} \hat{A}_{\rho}+\hat{A}_{\mu} * \partial_{\nu} \delta \hat{A}_{\rho}\right. \\
& \left.+\frac{2}{3}\left(\delta \hat{A}_{\mu} * \hat{A}_{\nu} * \hat{A}_{\rho}+\hat{A}_{\mu} * \delta \hat{A}_{\nu} * \hat{A}_{\rho}+\hat{A}_{\mu} * \hat{A}_{\nu} * \delta \hat{A}_{\rho}\right)\right)= \\
= & \frac{\kappa}{4 \pi} \operatorname{Tr} \int_{\mathcal{M}} d^{3} x \epsilon^{\mu \nu \rho}\left(\delta \hat{A}_{\mu} * \partial_{\nu} \hat{A}_{\rho}-\partial_{\nu} \hat{A}_{\mu} * \delta \hat{A}_{\rho}+\partial_{\nu}\left(\hat{A}_{\mu} * \delta \hat{A}_{\rho}\right)\right. \\
& \left.+\frac{2}{3}\left(\delta \hat{A}_{\mu} * \hat{A}_{\nu} * \hat{A}_{\rho}+\hat{A}_{\mu} * \delta \hat{A}_{\nu} * \hat{A}_{\rho}+\hat{A}_{\mu} * \hat{A}_{\nu} * \delta \hat{A}_{\rho}\right)\right)
\end{aligned}
$$

La derivada variacional es el coeficiente de $\delta \hat{A}_{\mu}$ en la variación de la acción. Para identificarla, es necesario reordenar los productos estrella en la expresión anterior, de manera de dejar $\delta \hat{A}_{\mu}$ a la izquierda, y luego eliminar la estrella que multiplica a $\delta \hat{A}_{\mu}$. Como se muestra en el apéndice 13, este tipo de manipulaciones del producto Moyal genera derivadas totales que contribuirán a la variación de la acción bajo la forma de términos de borde.

Siguiendo el apéndice, definiremos las dos derivadas totales que surgen al conmutar un producto de Moyal $\partial_{\alpha} B^{\alpha}\left[f_{1}, f_{2}\right]=\left[f_{1}, f_{2}\right]$ y al eliminar la estrella $\partial_{\alpha} \tilde{B}^{\alpha}\left[f_{1}, f_{2}\right]=f_{1} * f_{2}-f_{1} f_{2}$. Entonces, como primer paso, reordenaremos los términos en (7.1) de manera de dejar $\delta \hat{A}_{\mu}$ a la derecha, poniendo atención a los términos de borde que aparecen en el proceso

$$
\begin{aligned}
\delta S_{C S}^{\theta}[\hat{A}]= & \frac{\kappa}{4 \pi} \operatorname{Tr} \int_{\mathcal{M}} d^{3} x \epsilon^{\mu \nu \rho}\left(\delta \hat{A}_{\mu} * \partial_{\nu} \hat{A}_{\rho}-\delta \hat{A}_{\rho} * \partial_{\nu} \hat{A}_{\mu}+\partial_{\nu}\left(\hat{A}_{\mu} * \delta \hat{A}_{\rho}\right)-\partial_{\alpha} B^{\alpha}\left[\partial_{\nu} \hat{A}_{\mu}, \delta \hat{A}_{\rho}\right]\right. \\
& \left.+2 \delta \hat{A}_{\mu} * \hat{A}_{\nu} * \hat{A}_{\rho}+\frac{2}{3} \partial_{\alpha} B^{\alpha}\left[\hat{A}_{\mu}, \delta \hat{A}_{\nu} * \hat{A}_{\rho}\right]+\frac{2}{3} \partial_{\alpha} B^{\alpha}\left[\hat{A}_{\mu} * \hat{A}_{\nu}, \delta \hat{A}_{\rho}\right]\right)
\end{aligned}
$$

aquí, la derivada total en la primera línea aparece cuando se rota el término cuadrático en $\hat{A}_{\mu}$ y las dos derivadas totales en la segunda línea aparecen al hacer lo mismo con el término cúbico. La expresión resultante puede reacomodarse para dar

$$
\begin{aligned}
\delta S_{C S}^{\theta}[\hat{A}]= & \frac{\kappa}{4 \pi} \operatorname{Tr} \int_{\mathcal{M}} d^{3} x \epsilon^{\mu \nu \rho}\left(\delta \hat{A}_{\mu} * \hat{F}_{\nu \rho}+\partial_{\alpha}\left(\delta_{\nu}^{\alpha} \hat{A}_{\mu} * \delta \hat{A}_{\rho}-B^{\alpha}\left[\partial_{\nu} \hat{A}_{\mu}, \delta \hat{A}_{\rho}\right]\right.\right. \\
& \left.\left.+\frac{2}{3} B^{\alpha}\left[\hat{A}_{\mu}, \delta \hat{A}_{\nu} * \hat{A}_{\rho}\right]+\frac{2}{3} B^{\alpha}\left[\hat{A}_{\mu} * \hat{A}_{\nu}, \delta \hat{A}_{\rho}\right]\right)\right) .
\end{aligned}
$$


En esta última expresión, es necesario eliminar la estrella que multiplica a $\delta \hat{A}_{\mu}$, de manera de identificar correctamente la derivada variacional de la acción como el coeficiente correspondiente, con lo que se llega a la forma final

$$
\begin{aligned}
\delta S_{C S}^{\theta}[\hat{A}]= & \frac{\kappa}{4 \pi} \operatorname{Tr} \int_{\mathcal{M}} d^{3} x \epsilon^{\mu \nu \rho}\left(\delta \hat{A}_{\mu} \hat{F}_{\nu \rho}+\partial_{\alpha}\left(\tilde{B}^{\alpha}\left[\delta \hat{A}_{\mu}, \hat{F}_{\nu \rho}\right]+\delta_{\nu}^{\alpha} \hat{A}_{\mu} * \delta \hat{A}_{\rho}-B^{\alpha}\left[\partial_{\nu} \hat{A}_{\mu}, \delta \hat{A}_{\rho}\right]\right.\right. \\
& \left.\left.+\frac{2}{3} B^{\alpha}\left[\hat{A}_{\mu}, \delta \hat{A}_{\nu} * \hat{A}_{\rho}\right]+\frac{2}{3} B^{\alpha}\left[\hat{A}_{\mu} * \hat{A}_{\nu}, \delta \hat{A}_{\rho}\right]\right)\right)= \\
= & \frac{\kappa}{4 \pi} \operatorname{Tr} \int_{\mathcal{M}} d^{3} x \epsilon^{\mu \nu \rho} \delta \hat{A}_{\mu} \hat{F}_{\nu \rho}+\frac{\kappa}{4 \pi} \operatorname{Tr} \int_{\partial \mathcal{M}} d^{2} x n_{\alpha} B_{\text {total }}^{\alpha}
\end{aligned}
$$

donde hemos definido

$$
\begin{aligned}
n_{\alpha} B_{\text {total }}^{\alpha}= & n_{\alpha} \epsilon^{\mu \nu \rho}\left(\delta_{\nu}^{\alpha} \hat{A}_{\mu} * \delta \hat{A}_{\rho}+\tilde{B}^{\alpha}\left[\delta \hat{A}_{\mu}, \hat{F}_{\nu \rho}\right]-B^{\alpha}\left[\partial_{\nu} \hat{A}_{\mu}, \delta \hat{A}_{\rho}\right]+\frac{2}{3} B^{\alpha}\left[\hat{A}_{\mu}, \delta \hat{A}_{\nu} * \hat{A}_{\rho}\right]\right. \\
& \left.+\frac{2}{3} B^{\alpha}\left[\hat{A}_{\mu} * \hat{A}_{\nu}, \delta \hat{A}_{\rho}\right]\right)
\end{aligned}
$$

El procedimiento usual es imponer condiciones de contorno tales que anulen el término de borde en (7.4). De esta manera, la acción es diferenciable, es decir tiene una derivada variacional bien definida, y las ecuaciones de movimiento y las condiciones de contorno son

$$
\frac{\delta S_{C S}^{\theta}}{\delta \hat{A}_{\mu}}=\epsilon^{\mu \nu \rho} \hat{F}_{\nu \rho}=0,\left.\quad n_{\alpha} B^{\alpha}\right|_{\partial \mathcal{M}}=0 .
$$

Para hacer esto, necesitamos anular los términos de borde presentes en (7.5). En el caso usual conmutativo, en la expresión (7.5) subsiste solamente el primer término, que se hace cero con sólo imponer la nulidad de una de las componentes tangenciales del campo de gauge en la frontera. En el caso no conmutativo, surgen complicaciones debidas a la presencia de los términos que contienen $B^{\mu}[\cdot, \cdot]$ y $\tilde{B}^{\mu}[\cdot, \cdot]$.

Una primera posibilidad es que sobre alguno de los bordes del problema, el producto escalar $n_{\mu} B^{\mu}[\cdot, \cdot]$ y $n_{\mu} \tilde{B}^{\mu}[\cdot, \cdot]$ sea nulo. Para que esto suceda, dado que $B^{\mu}[\cdot, \cdot \cdot]$ es proporcional a $\theta^{\mu \nu}$, es necesario que el tensor $\theta^{\mu \nu}$ no tenga componentes en la dirección normal al borde $n_{\mu} \theta^{\mu \nu}=0$. Como primera observación, dado que $\theta^{\mu \nu}$ es constante, $n_{\mu}$ debe ser constante para poder cumplir esta condición, lo que implica que el borde es un plano. Además, esto significa que la dirección normal al borde es la direción conmutativa $x^{\prime 0}$ de la que hemos hablado en la sección 5.2, por lo tanto las direcciones tangenciales al borde serán ${x^{\prime}}^{1}$ y ${x^{\prime 2}}^{2}$. Por lo tanto en este caso la frontera es un plano perpendicular a la dirección conmutativa ${x^{\prime}}^{0}$. En tal caso, la expresión (7.5) se reduce a

$$
n_{\alpha} B^{\alpha}=n_{\alpha} \epsilon^{\mu \alpha \rho} \hat{A}_{\mu} * \delta \hat{A}_{\rho} .
$$


Haciendo una transformación de Lorentz al sistema de coordenadas $\left(x_{0}^{\prime}, x_{1}^{\prime}, x_{2}^{\prime}\right)$, en el cual $n_{\alpha}=(1,0,0)$, tenemos

$$
n_{\alpha} B^{\alpha}=\hat{A}_{1^{\prime}} * \delta \hat{A}_{2^{\prime}}-\hat{A}_{2^{\prime}} * \delta \hat{A}_{1^{\prime}}
$$

donde es fácil ver que si ponemos por ejemplo $\left.\hat{A}_{1^{\prime}}\right|_{\partial M}=0$, automáticamente todas las derivadas tangenciales de $\hat{A}_{1^{\prime}}$ se anulan en la frontera y, dado que en este caso el producto estrella no involucra derivadas normales, toda la expresión (7.8) se anula. Por lo tanto hemos llegado a la conclusión siguiente: Si existen bordes sobre los cuales $\theta^{\mu \nu}$ no tiene componentes normales, sobre ellos es necesario y suficiente para la diferenciabilidad de la acción, que se anule sólo una componente tangencial del campo de gauge. Ésta es la misma condición de contorno que en el caso conmutativo.

Sin embargo, en el caso general en el cual $\theta^{\mu \nu}$ tiene componentes en la dirección perpendicular a la frontera, los términos que contienen $B^{\mu}[\cdot, \cdot]$ y $\tilde{B}^{\mu}[\cdot, \cdot]$ no se anulan automáticamente, y es necesario imponer condiciones de contorno mas fuertes sobre el campo de gauge. Por ejemplo, centrándonos en el segundo término en (7.5)

$$
\epsilon^{\mu \nu \rho} \tilde{B}^{\alpha}\left[\delta \hat{A}_{\mu}, \hat{F}_{\nu \rho}\right]=2 \tilde{B}^{\alpha}\left[\delta \hat{A}_{0}, \hat{F}_{12}\right]+2 \tilde{B}^{\alpha}\left[\delta \hat{A}_{1}, \hat{F}_{20}\right]-2 \tilde{B}^{\alpha}\left[\delta \hat{A}_{2}, \hat{F}_{10}\right] .
$$

Como se puede ver en las fórmulas (13.3), algunos de estos términos se anulan cuando se impone que una de las funciones en el argumento se anule en el borde, junto con sus infinitas derivadas normales. Si en esta expresión, ponemos por ejemplo $\hat{A}_{0}$ y todas sus derivadas normales iguales a cero, obtenemos

$$
\epsilon^{\mu \nu \rho} \tilde{B}^{\alpha}\left[\delta \hat{A}_{\mu}, \hat{F}_{\nu \rho}\right]=-2 \tilde{B}^{\alpha}\left[\delta \hat{A}_{1}, \partial_{0} \hat{A}_{2}\right]+2 \tilde{B}^{\alpha}\left[\delta \hat{A}_{2}, \partial_{0} \hat{A}_{2}\right]
$$

donde aún hay contribuciones provenientes de las otras componentes del campo de gauge. Por lo tanto, debemos exigir la nulidad de una componente más, digamos $\hat{A}_{1}$, y de todas sus derivadas normales en la frontera [78]. Esto es suficiente para anular los otros términos en (7.5). Con lo cual concluimos que sobre aquellos bordes en los cuales $\theta^{\mu \nu}$ tenga componentes normales no nulas, la condición de contorno a imponer es que dos componentes del campo de gauge, junto con sus infinitas derivadas normales, sean cero.

\section{Simetrías que preservan las condiciones de contorno:}

Habiendo establecido las condiciones de contorno, el siguiente punto es encontrar, dentro del grupo de simetrías de la acción, el subgrupo de transformaciones que preservan estas condiciones de contorno. Este subgrupo será el grupo de simetrías del sistema.

En el primer caso particular que analizamos, es decir cuando $\theta^{\mu \nu}$ no tiene componentes transversales, la condición de contorno adecuada es anular una componente tangencial del campo de gauge en la frontera, digamos $\hat{A}_{1^{\prime}}$. Bajo un cambio de gauge, esta componente se transformará según

$$
\left.\delta \hat{A}_{1^{\prime}}\right|_{\partial \mathcal{M}}=\left.\left(\partial_{1^{\prime}} \lambda+\left[\hat{A}_{1^{\prime}}, \lambda\right]\right)\right|_{\partial \mathcal{M}} \equiv 0
$$


en esta fórmula, el conmutador es nulo porque sólo involucra derivadas tangenciales de $\hat{A}_{1^{\prime}}$, que son naturalmente nulas en la frontera. Por lo tanto, si $\lambda$ no depende de la dirección tangencial $x^{\prime 1}$ en la frontera, de manera que el primer término (7.11) sea cero, la transformación de gauge deja invariante la condición de contorno. Entonces si $\theta^{\mu \nu}$ no tiene componentes normales, las simetrías de la teoría estarán restringidas a las transformaciones $\hat{g}: \mathcal{M} \rightarrow G$ tales que $\left.\hat{g}\right|_{\partial \mathcal{M}}$ no dependa de $x^{\prime 1}$.

En el segundo caso que analizamos más arriba, es decir cuando $\theta^{\mu \nu}$ tiene componentes normales a la superficie, la condición de contorno adecuada será anular dos componentes del campo de gauge, digamos $\hat{A}_{0}$ y $\hat{A}_{1}$, y todas sus derivadas normales en el borde. Entonces bajo una transformación de gauge, estas componentes se transformarán de acuerdo con

$$
\left.\delta \hat{A}_{0}\right|_{\partial \mathcal{M}}=\left.\left(\partial_{0} \lambda+\left[\hat{A}_{0}, \lambda\right]\right)\right|_{\partial \mathcal{M}} \equiv 0
$$

y similar para $\hat{A}_{1}$. Para que esta transformación no afecte las condiciones de contorno, deberá cumplirse que $\delta \hat{A}_{0}, \delta \hat{A}_{1}$ y todas sus derivadas normales se anulan en la superficie. En primer lugar, para anular $\delta \hat{A}_{0}$ y $\delta \hat{A}_{1}$ basta con que $\lambda$ no dependa de $x_{0}$ ni de $x_{1}$ en la superficie lo que hace cero el primer término en (7.12). En cuanto al segundo término, es cero debido a la nulidad de todas las derivadas normales de $\hat{A}_{0}$ y $\hat{A}_{1}$. Para preservar la nulidad de las derivadas normales de $\delta \hat{A}_{0}$ y $\delta \hat{A}_{1}$ debe cumplirse que

$$
\left.\partial_{n}^{(q)} \delta \hat{A}_{0}\right|_{\partial \mathcal{M}}=\left.\left(\partial_{n}^{(q)} \partial_{0} \lambda+\left[\hat{A}_{0}, \partial_{n}^{(q)} \lambda\right]\right)\right|_{\partial \mathcal{M}} \equiv 0,
$$

y lo mismo para $A_{1}$. Por lo tanto, es necesario y suficiente que todas las derivadas normales de $\lambda$ se anulen en la superficie. Entonces en el caso en el que $\theta^{\mu \nu}$ tiene componentes no nulas en las direcciones normales a la superficie, las transformaciones de simetría serán aquellas tales que $\left.\hat{g}\right|_{\partial \mathcal{M}}$ no dependa de $x_{0}, x_{1}$ y que $\left.\partial_{n}^{(q)} \hat{g}\right|_{\partial \mathcal{M}}=0$.

\subsection{La conexión entre la acción de Chern-Simons y la acción quiral de Wess-Zumino-Witten}

Como es bien conocido, la teoría de Chern-Simons conmutativa se puede reducir a una acción efectiva que tome en cuenta solamente los grados de libertad en el borde de la variedad sobre la cual están definidos los campos, siguiendo diferentes caminos [25]-[33]. Aquí discutiremos de qué manera se puede establecer esta conexión en el caso no conmutativo.

Si reescribimos la acción de Chern-Simons no conmutativa, singularizando una componente del campo de gauge, digamos $\hat{A}_{0}$, llegamos a la siguiente expresión

$$
\begin{aligned}
& S_{C S}^{\theta}\left[\hat{A}_{0}, \hat{A}_{i}\right]=\frac{\kappa}{4 \pi} \operatorname{Tr} \int_{\mathcal{M}} d^{3} x\left(\epsilon^{i j}\left(\hat{A}_{0} * \partial_{i} \hat{A}_{j}+\frac{2}{3} \hat{A}_{0} * \hat{A}_{i} * \hat{A}_{j}\right)\right. \\
&\left.-\epsilon^{i j}\left(\hat{A}_{i} * \partial_{0} \hat{A}_{j}+\frac{2}{3} \hat{A}_{i} * \hat{A}_{0} * \hat{A}_{j}\right)+\epsilon^{i j}\left(\hat{A}_{i} * \partial_{j} \hat{A}_{0}+\frac{2}{3} \hat{A}_{i} * \hat{A}_{j} * \hat{A}_{0}\right)\right)(
\end{aligned}
$$


lo que se puede transformar mediante rotación de productos estrella e integración por partes en

$$
S_{C S}^{\theta}\left[\hat{A}_{0}, \hat{A}_{i}\right]=\frac{\kappa}{4 \pi} \operatorname{Tr} \int_{\mathcal{M}} d^{3} x \epsilon^{i j}\left(\hat{A}_{0} * \hat{F}_{i j}-\hat{A}_{i} * \partial_{0} \hat{A}_{j}\right)+\frac{\kappa}{4 \pi} \operatorname{Tr} \int_{\partial \mathcal{M}} d^{2} x n_{\alpha} B_{\text {total }}^{\alpha},
$$

donde el término de borde, que surge de las rotaciones y de la integración por partes, es similar al que hemos visto en la sección anterior y viene dado por

$$
B_{\text {total }}^{\alpha}=\epsilon^{i j}\left(\delta_{\alpha j} \hat{A}_{i} * \hat{A}_{0}+B^{\alpha}\left[\partial_{j} \hat{A}_{i}, \hat{A}_{0}\right]+\frac{2}{3}\left(B^{\alpha}\left[\hat{A}_{i} * \hat{A}_{j}, \hat{A}_{0}\right]-B^{\alpha}\left[\hat{A}_{i}, \hat{A}_{0} * \hat{A}_{j}\right]\right)\right) .
$$

Estos términos se anulan para cualquiera de las dos condiciones de contorno que hemos explicado en la sección previa. Si $\theta^{\mu \nu}$ no tiene componentes normales a la superficie, entonces los términos $B^{\alpha}[\cdot, \cdot]$ son naturalmente nulos y el término restante se anula si $\left.\hat{A}_{0}\right|_{\partial \mathcal{M}}=0$. Por otro lado, si $\theta^{\mu \nu}$ tiene componentes normales, las condiciones de contorno adecuadas exigen que dos cualesquiera componentes del campo de gauge se anulen en la frontera junto con todas sus derivadas normales, lo que hace que toda la expresión anterior se anule.

Si ahora además eliminamos la estrella en el primer término de la acción (7.15), el correspondiente término de borde también se anula, dejándonos con la expresión simplificada

$$
S_{C S}^{\theta}\left[\hat{A}_{0}, \hat{A}_{i}\right]=\frac{\kappa}{4 \pi} \operatorname{Tr} \int_{\mathcal{M}} d^{3} x \epsilon^{i j}\left(\hat{A}_{0} \hat{F}_{i j}-\hat{A}_{i} * \partial_{0} \hat{A}_{j}\right) .
$$

Por conveniencia, por el momento mantendremos la estrella en el segundo término, si bien se podría eliminar en razón de nuestras condiciones de borde.

Usando la acción (7.17), la función de partición para la teoría de Chern-Simons no conmutativa toma la forma

$$
Z=\int \mathcal{D} \hat{A}_{i} \mathcal{D} \hat{A}_{0} e^{S_{C S}^{\theta}\left[\hat{A}_{0}, \hat{A}_{i}\right]}
$$

Para cada uno de los puntos de $\mathcal{M}, \hat{A}_{0}$ actúa como un multiplicador de Lagrange, forzando la anulación de las componentes espaciales de la conexión

$$
\epsilon^{i j} \hat{F}_{i j}=0
$$

entonces, la función de partición (7.18) se puede reescribir como

$$
Z=\int \mathcal{D} \hat{A}_{i} \delta\left(\epsilon^{i j} \hat{F}_{i j}\right) \exp \left(-\frac{i \kappa}{4 \pi} \operatorname{Tr} \int_{\mathcal{M}} d^{3} x \epsilon^{i j} \hat{A}_{i} * \partial_{0} \hat{A}_{j}\right)
$$

El siguiente paso es resolver la ecuación (7.19) y substituir el resultado en el exponente de (7.20). Para esto es necesario definir la topología de nuestra variedad. En lo que sigue, nos limitaremos al caso $\mathcal{M}=\Sigma \times \mathbb{R}$ donde $\Sigma$ tiene la topología del disco. Entonces la solución 
de la condición de conexión plana (7.19) es $\hat{A}_{i}=\hat{g} * \partial_{i} \hat{g}^{-1}$, con $\hat{g}: \mathcal{M} \rightarrow G$ una función arbitraria. Reinsertando en (7.20) se tiene

$$
Z=\int \mathcal{D} \hat{g} e^{i S_{Q W Z W}^{\theta}[\hat{g}]}
$$

donde $S_{Q W Z W}^{\theta}[\hat{g}]$ es una acción que tiene la forma de un modelo de Wess-Zumino-Witten quiral no conmutativo

$$
\begin{aligned}
S_{Q W Z W}^{\theta}[\hat{g}]= & -\frac{\kappa}{4 \pi} \operatorname{Tr} \int_{\partial \mathcal{M}} d^{2} x \check{t}^{i}\left(\hat{g} * \partial_{0} \hat{g}^{-1}\right) *\left(\hat{g} * \partial_{i} \hat{g}^{-1}\right) \\
& -\frac{\kappa}{12 \pi} \operatorname{Tr} \int_{\mathcal{M}} d^{3} x \epsilon^{\mu \nu \rho}\left(\hat{g} * \partial_{\mu} \hat{g}^{-1}\right) *\left(\hat{g} * \partial_{\nu} \hat{g}^{-1}\right) *\left(\hat{g} * \partial_{\rho} \hat{g}^{-1}\right),
\end{aligned}
$$

aquí $\check{t}^{i}$ es un vector unitario tangente al borde de $\Sigma$. Nótese que debido a la presencia del producto estrella, en un borde arbitrario no es posible combinar $\check{t}_{i}$ con $\partial_{i} \hat{g}$ para formar la derivada tangencial de $\hat{g}$. Esto es posible solamente en un borde plano, donde $\check{t}_{i}$ es constante, y en ese caso se tiene

$$
\begin{aligned}
S_{Q W Z W}^{\theta}[\hat{g}]= & -\frac{\kappa}{4 \pi} \operatorname{Tr} \int_{\partial \mathcal{M}} d^{2} x\left(\hat{g} * \partial_{0} \hat{g}^{-1}\right) *\left(\hat{g} * \partial_{\varphi} \hat{g}^{-1}\right) \\
& -\frac{\kappa}{12 \pi} \operatorname{Tr} \int_{\mathcal{M}} d^{3} x \epsilon^{\mu \nu \rho}\left(\hat{g} * \partial_{\mu} \hat{g}^{-1}\right) *\left(\hat{g} * \partial_{\nu} \hat{g}^{-1}\right) *\left(\hat{g} * \partial_{\rho} \hat{g}^{-1}\right),
\end{aligned}
$$

Nótese que, si $\theta^{\mu \nu}$ tiene componentes normales al borde de la variedad, la acción (7.23) no es la acción quiral de Wess-Zumino-Witten no conmutativa definido sobre $\partial \Sigma \times \mathbb{R}$, ya que contiene infinitas derivadas de los campos $\hat{g}$ en las direcciones que salen de $\partial \Sigma \times \mathbb{R}$. Entonces sólo en el caso en el que $\theta^{\mu \nu}$ no tenga componentes normales a la frontera, la expresión (7.23) corresponde a la acción quiral de Wess-Zumino-Witten no conmutativa en $\partial \Sigma \times \mathbb{R}$.

En el caso general en el que $\theta^{\mu \nu}$ tiene componentes normales a la frontera, la condición de contorno sobre el campo de gauge impone que dos componentes del campo se anulen en la superficie. Por lo tanto en el primer término de la fórmula (7.22) debemos reemplazar $\hat{A}_{1}=\hat{g} * \partial_{1} \hat{g}^{-1}$ por cero, y sólo sobrevive $\hat{A}_{2}=\hat{g} * \partial_{2} \hat{g}^{-1}$, por lo que la expresión se reduce a

$$
\begin{aligned}
\hat{S}_{C W Z W}[\hat{g}]= & -\frac{\kappa}{4 \pi} \operatorname{Tr} \int_{\partial \mathcal{M}} d^{2} x \check{t}^{2}\left(\hat{g} * \partial_{0} \hat{g}^{-1}\right) *\left(\hat{g} * \partial_{2} \hat{g}^{-1}\right) \\
& -\frac{\kappa}{12 \pi} \operatorname{Tr} \int_{\mathcal{M}} d^{3} x \epsilon^{\mu \nu \rho}\left(\hat{g} * \partial_{\mu} \hat{g}^{-1}\right) *\left(\hat{g} * \partial_{\nu} \hat{g}^{-1}\right) *\left(\hat{g} * \partial_{\rho} \hat{g}^{-1}\right) .
\end{aligned}
$$

Donde en el término de borde sólo interviene la derivada en la direción $x^{2}$, mostrando explícitamente la ruptura de la invarianza de Lorentz introducida por la no conmutatividad. Entonces en el caso general de borde curvo, la acción de Chern-Simons es equivalente a la acción (7.24), donde sólo permanece una de las derivadas en el término cuadrático. 


\subsection{Alcances y limitaciones del cálculo}

Algunas consideraciones acerca de la validez de nuestros cálculos

- Existencia del producto estrella: Hemos utilizado en este capítulo la expresión diferencial del producto de Moyal (2.2). Cuando realizamos el producto entre dos funciones a cualquier orden finito en $\theta^{\mu \nu}$, la serie en derivadas truncada de este producto es siempre una expresión local, por lo que siempre obtenemos como resultado una función continua bien definida sobre nuestra variedad.

Sin embargo, al sumar los infinitos órdenes en el desarrollo del producto, podemos encontrarnos con que el resultado no es una función continua bien definida sobre nuestra variedad. Esto se hace evidente cuando escribimos la forma integral del producto estrella, la cual claramente depende de la variedad elegida.

- Existencia de funciones que cumplan las condiciones de contorno: Otro punto a tener en cuenta, es la existencia de funciones no triviales que verifiquen las condiciones de contorno (7.12). Debido a que se trata de condiciones de contorno muy fuertes, no es en principio evidente que existan tales funciones.

Ambas cuestiones han sido analizadas en profundidad y respondidas afirmativamente en la referencia [78].

\subsection{Conclusiones}

Como resumen de las conclusiones podemos decir que

- La acción de Chern-Simons no conmutativa definida en un variedad con borde es diferenciable e invariante de gauge siempre que se cumpla alguna de las siguientes condiciones de borde, dependiendo de las direcciones del parámetro $\theta^{\mu \nu}$

1. Caso especial: si existen bordes sobre los cuales $\theta^{\mu \nu}$ no tiene componentes normales, sobre ellos es necesario y suficiente para la diferenciabilidad de la acción que se anule una componente tangencial del campo de gauge.

2. Caso general: sobre aquellos bordes en los cuales $\theta^{\mu \nu}$ tenga componentes normales no nulas, la condición de contorno a imponer es que $\operatorname{dos}^{2}$ componentes del campo de gauge, junto con sus infinitas derivadas normales, sean cero.

- Las transformaciones de simetría que preservan las condiciones de contorno serán aquellas que cumplan las adecuadas condiciones de borde, a saber

${ }^{2} \mathrm{El}$ hecho de que se debe imponer la condiciones de contorno sobre dos componentes del campo de gauge en lugar de sobre una sola fue señalado originalmente en [78] 
1. Caso especial: si $\theta^{\mu \nu}$ no tiene componentes longitudinales, las simetrías de la teoría serán las funciones $\hat{g}: \mathcal{M} \rightarrow G$ tales que $\left.\hat{g}\right|_{\partial \mathcal{M}}$ no dependa de una dirección tangente $x^{\prime 1}$ en el borde.

2. Caso general: en el caso en que $\theta^{\mu \nu}$ tenga componentes no nulas en las direcciones normales a la superficie, las transformaciones de simetría serán aquellas tales que $\left.\hat{g}\right|_{\partial \mathcal{M}}$ no dependa de $x_{0}, x_{1}$ y que $\left.\partial_{n}^{(q)} \hat{g}\right|_{\partial \mathcal{M}}=0$ en el borde.

- Para el caso $\mathcal{M}=\Sigma \times \mathbb{R}$ donde $\Sigma$ tiene la topología del disco, la acción de Chern-Simons es equivalente a un modelo de Wess-Zumino-Witten con las siguientes características

1. Caso especial: cuando $\theta^{\mu \nu}$ no tiene componentes normales al borde del disco, obtenemos una acción quiral de Wess-Zumino-Witten, donde los productos se han transformado en productos estrella. Es decir obtenemos lo que llamaríamos un modelo quiral de Wess-Zumino-Witten no conmutativo.

2. Caso general: cuando $\theta^{\mu \nu}$ posee componentes no nulas normales al borde del disco, obtenemos un modelo de Wess-Zumino-Witten no conmutativo un poco más complicado, como se puede ver en la fórmula (7.24). Esto se debe a la necesidad de imponer condiciones de contorno sobre dos componentes del campo de gauge en lugar de sobre una sola. 


\section{Capítulo 8}

\section{Comportamiento de la acción de Chern-Simons bajo el mapeo de Seiberg-Witten}

En este capítulo estudiaremos el comportamiento de la acción de Chern-Simons no conmutativa definida en los capítulos anteriores, respecto del mapeo de Seiberg y Witten, que relaciona teorías de gauge conmutativas y no conmutativas. Como resultado importante veremos que su imagen bajo este mapeo es la acción de Chern-Simons usual conmutativa, en contraste con lo que sucede con otras teorías de gauge

\subsection{Algunas motivaciones}

En el capítulo precedente, hemos encontrado la relación entre la acción de Chern-Simons no conmutativa y la acción no conmutativa de Wess-Zumino-Witten quiral.

La teoría de Wess-Zumino-Witten no conmutativa ha sido estudiada en [83], donde se encontró un cambio de variables, similar al mapeo de Seiberg y Witten, que relaciona los campos $\hat{g}$ de un modelo de Wess-Zumino-Witten no conmutativo con los campos $g$ de un dado modelo conmutativo. Según los razonamientos expuestos en ese trabajo, la dinámica de este último está regida por la acción de Wess-Zumino-Witten conmutativa. Por lo tanto, en el caso espacial de la teoría de Wess-Zumino-Witten, el cambio de variables mapea el modelo no conmutativo en el correspondiente modelo conmutativo. Esto no es automático en un modelo general, por ejemplo, al aplicar el mapeo de Seiberg y Witten (4.22) para los campos de gauge $\hat{A}_{\mu}$ a la acción de Yang-Mills no conmutativa, se obtiene una acción complicada para los campos conmutativos $A_{\mu}$, que tiene la forma de un desarrollo en potencias de $\theta^{\mu \nu}$. Es decir que el cambio de variables no mapea la acción de Yang-Mills no conmutativa en la acción de Yang-Mills conmutativa.

El cambio de variables para $\hat{g}$ definido en [83] se puede aplicar también al modelo quiral de 
Wess-Zumino-Witten no conmutativo, y es razonable suponer que la mencionada conexión se verificará también para ese modelo, obteniéndose para $g$ una acción quiral de Wess-ZuminoWitten conmutativa. En ese caso, podemos adelantar una conexión análoga para las teorías de Chern-Simons. La situación se puede visualizar en el siguiente esquema

$$
\begin{array}{ccc}
S_{Q W Z W}^{\theta}[\hat{g}] & \longleftrightarrow & S_{C S}^{\theta}[\hat{A}] \\
\uparrow & \uparrow ? \\
S_{Q W Z W}[g] & \longleftrightarrow & S_{C S}[A]
\end{array}
$$

La acción de Chern-Simons conmutativa está relacionada con el modelo de Wess-ZuminoWitten quiral conmutativo por integración del $A_{0}$ como hemos mencionado en el capítulo 5 . La misma conexíon se verifica para los modelos no conmutativos como demostramos en el capítulo precedente. Por otro lado, el modelo quiral de Wess-Zumino-Witten no conmutativo se mapea a través del cambio de variables definido en [83] en el modelo conmutativo. Entonces podemos inferir que lo mismo sucede para las acciones de Chern-Simons.

Pero para estudiar esta última conexión, no es necesario recurrir al mapeo definido en [83] para los campos $\hat{g}$ del modelo de Wess-Zumino-Witten. Podemos utilizar directamente el mapeo de Sieberg y Witten (4.22) para los campos de gauge $\hat{A}_{\mu}$, e investigar si la acción de Chern-Simons no conmutativa se mapea en la acción de Chern-Simons conmutativa.

Otra evidencia en favor de esta hipótesis se obtiene al aplicar el mapeo de Seiberg y Witten a una configuración clásica de la teoría de Chern-Simons no conmutativa, que cumple las ecuaciones de movimiento $\hat{F}_{\mu \nu}=0$. De acuerdo a la regla de transfomación para $\hat{F}_{\mu \nu}$, ecuación 4.23 , vemos que la correspondiente teoría conmutativa cumple $F_{\mu \nu}=0$, con lo que tenemos el siguiente esquema

$$
\begin{array}{ccc}
\hat{F}_{\mu \nu}=0 & \longleftrightarrow & S_{C S}^{\theta}[\hat{A}] \\
\uparrow & \uparrow ? \\
F_{\mu \nu}=0 & \longleftrightarrow & S_{C S}[A]
\end{array}
$$

Entonces soluciones clásicas de la teoría de Chern-Simons no conmutativa son mapeadas en soluciones clásicas de la teoría de Chern-Simons conmutativa.

\section{2. $\quad$ El mapeo de Seiberg y Witten aplicado a la acción de Chern-Simons}

En lo que sigue aplicaremos el mapeo de Seiberg y Witten a la acción de Chern-Simons no conmutativa, para comprobar si se verifica la relación sugerida más arriba. La teoría de 
Chern-Simons estará definida sobre una variedad arbitraria $\mathcal{M}$ y supondremos que se cumplen las condiciones de contorno adecuadas para que la acción sea diferenciable e invariante de gauge.

Nótese que quedan descartadas las condiciones de contorno del caso especial que hemos discutido en el capítulo anterior, donde $\theta^{\mu \nu}$ no tiene componentes normales al borde, ya que nos proponemos estudiar el comportamiento de la acción de Chern-Simons bajo variaciones totalmente arbitrarias del parámetro antisimétrico $\theta^{\mu \nu}$.

Tomaremos como punto de partida la acción de Chern-Simons en la forma (7.17), en la cual sacamos el producto estrella en el segundo término, lo que no genera términos de borde debido a las condiciones de contorno, es decir que tenemos que

$$
S_{C S}^{\theta}\left[\hat{A}_{0}, \hat{A}_{i}\right]=\frac{\kappa}{4 \pi} \operatorname{Tr} \int_{\mathcal{M}} d^{3} x \epsilon^{i j}\left(\hat{A}_{0} \hat{F}_{i j}-\hat{A}_{i} \partial_{0} \hat{A}_{j}\right) .
$$

Como esta expresión no contiene productos estrella, depende del parámetro de no conmutatividad $\theta^{\mu \nu}$ solamente a través de la dependencia de $\hat{A}_{\mu}$ dada por el mapeo de Seiberg y Witten. Entonces, si derivamos con respecto a $\theta_{\mu \nu}$ obtenemos

$$
\begin{aligned}
\frac{\partial S_{C S}^{\theta}(\hat{A})}{\partial \theta_{\mu \nu}} & =\frac{\kappa}{4 \pi} \int_{\mathcal{M}} d^{3} x \epsilon^{i j} \frac{\partial}{\partial \theta_{\mu \nu}}\left(\hat{A}_{0} \hat{F}_{i j}-A_{i} \partial_{0} A_{j}\right)= \\
& =\frac{\kappa}{4 \pi} \int_{\mathcal{M}} d^{3} x \epsilon^{i j}\left(\frac{\partial \hat{A}_{0}}{\partial \theta_{\mu \nu}} \hat{F}_{i j}+\hat{A}_{0} \frac{\partial \hat{F}_{i j}}{\partial \theta_{\mu \nu}}-2 \frac{\partial A_{i}}{\partial \theta_{\mu \nu}} \partial_{0} A_{j}\right),
\end{aligned}
$$

donde para reescribir el último término hemos integrado por partes, descartando un término de superficie que se anula con las condiciones de contorno que hemos elegido. En la expresión precedente, las derivadas de los campos con respecto a $\theta^{\mu \nu}$ se obtienen de las fórmulas (4.22)

$$
\begin{aligned}
\frac{\partial \hat{A}_{\mu}}{\partial \theta^{\rho \sigma}} & =\frac{1}{4}\left\{\hat{A}_{\rho}, \partial_{\sigma} \hat{A}_{\mu}+\hat{F}_{\sigma \mu}\right\} \\
\frac{\partial \hat{F}_{\mu \nu}}{\partial \theta^{\rho \sigma}} & =\frac{1}{4}\left(-2\left\{\hat{F}_{\mu \rho}, \hat{F}_{\nu \sigma}\right\}+\left\{\hat{A}_{\rho}, \hat{D}_{\sigma} \hat{F}_{\mu \nu}+\partial_{\sigma} \hat{F}_{\mu \nu}\right\}\right) .
\end{aligned}
$$

Reemplazando (8.5) en la expresión (8.4) obtenemos una expresión para la variación de la acción bajo un cambio arbitrario de $\theta^{\mu \nu}$.

Mediante un cálculo directo, que no vale la pena detallar aquí, en el cual se deben mantener solamente los términos antisimétricos con respecto a los índices $\mu, \nu$ e $i, j$, obtenemos como resultado final

$$
\frac{\partial S_{C S}^{\theta}(\hat{A})}{\partial \theta_{\mu \nu}}=0 \quad \Rightarrow \quad S_{C S}^{\theta}(\hat{A})=S_{C S}(A),
$$

donde $S_{C S}(A)$ es la acción de Chern-Simons ordinaria conmutativa como se definición en el capítulo 5. Los pasos intermedios de este cálculo contienen integraciones por partes, las 
cuales generan términos de superficie, que se anulan debido a las condiciones de contorno. Es interesante notar que en el caso $U(1)$ el mapeo de Seiberg-Witten cancela los términos cúbicos presentes en $\hat{S}_{C S}(\hat{A})$.

En suma, hemos visto que la transformación de (8.5) mapea la acción de Chern-Simons no conmutativa en su contraparte conmutativa

\subsection{Alcances y limitaciones del cálculo}

Detallaremos a continuacion los alcances de nuestro cálculo, las posibles derivaciones y algunas de sus limitaciones

- Si bien entre las motivaciones originales de nuestro cálculo se encontraba la relación de la acción de Chern-Simons con el modelo quiral de Wess-Zumino-Witten y la conexión entre las acciones conmutativa y no conmutativa de éste último, nuestra demostración es completamente independiente de estas consideraciones, ya que involucra solamente a la acción de Chern-Simons y al cambio de variables (4.22) de Seiberg y Witten.

- Otra de las motivaciones fue la correspondencia de las soluciones clásicas de las teorías de Chern-Simons conmutativa y no conmutativa. Sin embargo, debemos señalar que nuestra demostración en ningún punto supone que los campos cumplen las ecuaciones de movimiento.

- El resultado obtenido es en realidad más general: la acción de Chern-Simons no conmutativa con un valor dado para el parámetro de no conmutatividad $\theta^{\mu \nu}$ se mapea por el mapeo de Seiberg y Witten en la acción no conmutativa con un valor diferente de este parámetro.

- Estos resultados pueden ser útiles al interpretar el esquema 8.1 en la otra dirección, como un argumento a favor de la hipótesis de que el mapeo definido en [83] relaciona también los modelos quirales de Wess-Zumino-Witten conmutativo y no conmutativo.

- Otra posible derivación de este resultado se infiere a partir del esquema 8.2: sería interesante estudiar el efecto del mapeo de Seiberg y Witten sobre otras teorías de gauge no conmutativas cuya ecuación clásica de movimiento sea $\hat{F}_{\mu \nu}=0$, como por ejemplo los modelos $B F$, así como también sobre otros modelos topológicos más generales.

- Este cálculo tiene aplicación solamente a la teoría de Chern-Simons clásica. Un análisis cuántico requeriría del estudio del cambio bajo el mapeo de Seiberg y Witten de la medida de integración $\mathcal{D} A_{\mu}$, con el gauge adecuadamente fijado. Debido a que este mapeo es no lineal, es difícil definir consistentemente el correspondiente jacobiano. 


\subsection{Conclusiones}

Resumimos a continuación las conclusiones de este capítulo

- La principal conclusión de este capítulo es que la acción de Chern-Simons no conmutativa se mapea por la transformación de Seiberg y Witten en la acción de Chern-Simons conmutativa. Esta es una propiedad no trivial de este modelo, que no se verifica en otras teorías como por ejemplo la de Maxwell o la de Yang-Mills.

- Esta conclusión se puede extender al resultado más general de que la acción de ChernSimons no conmutativa no depende del valor del parámetro $\theta^{\mu \nu}$, cuando se consideran a los campos de gauge $\hat{A}_{\mu}$ como funciones de este parámetro dadas por el mapeo de Seiberg y Witten. 


\section{Capítulo 9}

\section{Gravedad no conmutativa en tres dimensiones}

Formulamos una teoría de gravedad en tres dimensiones no conmutativas mediante el uso de la conexión entre gravedad tridimensional y teoría de Chern-Simons. En el sector euclídeo, consideramos, con constante cosmológica negativa, estudiaremos el efecto particular de la topología $T^{2} \times \mathbb{R}$ y mostramos que el agujero negro tridimensional (BTZ) resuelve las ecuaciones de movimiento no conmutativas.

\subsection{Teoría de Chern-Simons no conmutativa}

Como hemos explicado con cierto detalle en el capítulo 5, en el caso usual conmutativo, la gravitación en espacio euclídeo con constante cosmológica negativa, es completamente equivalente a una teoría de gauge, con grupo de gauge $S L(2, \mathbb{C})$, y cuya dinámica está regida por la acción de Chern-Simons.

En el caso no conmutativo, definiremos nuestra teoría de gravedad en $d=3$ dimensiones euclídeas y con constante cosmológica negativa, como la correspondiente teoría de gauge no conmutativa [126]. Sin embargo, como hemos mencionado en la sección 4.2, el álgebra de $S L(2, \mathbb{C})$ no es cerrada cuando los conmutadores se calculan usando el producto de Moyal, por lo que es necesario ampliarla y considerar el grupo $G L(2, \mathbb{C})$. El álgebra de Lie de $G L(2, \mathbb{C})$ esta generada con coeficientes complejos por la base $\left\{J_{a}, i I\right\}$, donde $J_{a}$ son los generadores que hemos definido en el capítulo 5. Una representación de estos generadores esta dada por $J_{1}=i \sigma_{1}, J_{2}=i \sigma_{2}, J_{3}=i \sigma_{3}$ donde $\sigma_{a}$ son las matrices de Pauli.

El campo de gauge $\hat{A}_{\mu} \in G L(2, \mathbb{C})$ se puede expandir en esta base de la siguiente manera

$$
\hat{A}_{\mu}=\hat{A}_{\mu}^{a} J_{a}+\hat{\mathbb{A}}_{\mu} i I=\hat{\mathrm{A}}_{\mu}+i \hat{\mathbb{A}}_{\mu}
$$

donde hemos llamado $\hat{\mathrm{A}}_{\mu}=A_{\mu}^{a} J_{a}$ a la parte $S L(2, \mathbb{C})$ de la conexión y $\hat{\mathbb{A}}_{\mu}$ a la parte $U(1)$. Dado que $\hat{A}_{\mu}$ es una conexión compleja, debemos definir un segundo campo independiente 
$\hat{\bar{A}}_{\mu}$.

$$
\hat{\bar{A}}_{\mu}=\hat{\bar{A}}_{\mu}^{a} J_{a}+\hat{\overline{\mathbb{A}}}_{\mu} i I=\hat{\overline{\mathrm{A}}}_{\mu}+i \hat{\overline{\mathbb{A}}}_{\mu}
$$

La acción adecuada no conmutativa para estos campos de gauge se define como

$$
S_{C S}^{\theta}\left[\hat{A}_{\mu}, \hat{\bar{A}}_{\mu}\right]=i\left(S_{C S}^{\theta}\left[\hat{A}_{\mu}\right]-\hat{S}_{C S}^{\theta}\left[\hat{\bar{A}}_{\mu}\right]\right)
$$

donde el factor $i$ proviene de la rotación de Wick necesaria para llevar la teoría de gravitación a espacio euclídeo. Es decir que tenemos dos teorías de gauge desacopladas, con acción de Chern-Simons no conmutativa, para los campos de gauge complejos $\hat{A}_{\mu}$ y $\hat{\bar{A}}_{\mu}$.

Para asegurar la diferenciabilidad de la acción, debemos imponer alguna de las dos condiciones de contorno estudiadas en el capítulo 7. En cualquiera de esos casos, las ecuaciones de movimiento son

$$
\hat{F}_{\mu \nu}\left[\hat{A}_{\rho}\right]=\hat{F}_{\mu \nu}\left[\hat{\bar{A}}_{\rho}\right]=0 .
$$

Las mismas condiciones de contorno nos garantizan que la acción es invariante frente a transformaciones de gauge no conmutativas. Si en las ecuaciones (9.4) separamos el campo $U(1), \hat{\mathbb{A}}_{\mu}$, del resto de las componentes, obtenemos

$$
\begin{aligned}
\hat{\mathrm{F}}_{\mu \nu}\left[\hat{\mathrm{A}}_{\rho}\right] & \equiv \partial_{\mu} \hat{\mathrm{A}}_{\nu}-\partial_{\nu} \hat{\mathrm{A}}_{\mu}-\frac{1}{2} \epsilon^{a b c}\left\{A_{\mu}^{b}, A_{\nu}^{c}\right\} J_{a}=i\left[\hat{\mathbb{A}}_{\mu}, \hat{\mathrm{A}}_{\nu}\right]+i\left[\hat{\mathrm{A}}_{\mu}, \hat{\mathbb{A}}_{\nu}\right] \\
\hat{\mathbb{F}}_{\mu \nu}\left[\mathbb{A}_{\rho}\right] & \equiv \partial_{\mu} \hat{\mathbb{A}}_{\nu}-\partial_{\nu} \hat{\mathbb{A}}_{\mu}+i\left[\hat{\mathbb{A}}_{\mu}, \hat{\mathbb{A}}_{\nu}\right]=i \operatorname{Tr}\left[\mathrm{A}_{\mu}, \mathrm{A}_{\nu}\right]
\end{aligned}
$$

y ecuaciones análogas para $\hat{\bar{A}}_{\mu}$. El miembro derecho de estas ecuaciones se anula para $\theta^{\mu \nu}=0$, mostrando que $\hat{\mathrm{A}}_{\mu}$ y $\hat{\mathbb{A}}_{\mu}$ se desacoplan en el límite conmutativo. Es importante notar que $\hat{\mathrm{F}}_{\mu \nu}$ no son las componentes en $J_{a}$ del tensor de curvatura $\hat{F}_{\mu \nu}$ de la teoría de gauge $G L(2, \mathbb{C})$, sino las que corresponderían a una teoría $S L(2, \mathbb{C})$, escrita en componentes. Análogamente, $\hat{\mathbb{F}}_{\mu \nu}$ no es la componente en la identidad de la curvatura $G L(2, \mathbb{C})$, sino la curvatura correspondiente a una teoría $U(1)$ no conmutativa. Para referencia futura, mencionaremos aquí que existen soluciones "planas en $S L(2 \mathbb{C})$ ", es decir con $\hat{\mathrm{F}}_{\mu \nu}=0$ siempre que,

$$
\left[\hat{\mathbb{A}}_{\mu}, \hat{\mathrm{A}}_{\nu}\right]+\left[\hat{\mathrm{A}}_{\mu}, \hat{\mathbb{A}}_{\nu}\right]=0
$$

En el límite $\theta^{\mu \nu} \rightarrow 0$, la teoría de gauge que hemos definido es equivalente a nivel clásico a la gravitación en $d=3$ dimensiones, más dos campos de gauge $U(1)$ libres y con acción de Chern-Simons. En efecto, los campos abelianos $\hat{\mathbb{A}}_{\mu}$ y $\hat{\overline{\mathbb{A}}}_{\mu}$ se desacoplan completamente de $\hat{\mathrm{A}}_{\mu}$ y $\hat{\overline{\mathrm{A}}}_{\mu}$ en ese límite, como se puede ver en (9.5-9.6), y pueden elegirse nulos. Si bien los campos se acoplan no trivialmente en el caso $\theta^{\mu \nu}$ finito, existen, como veremos, soluciones $\operatorname{con} \hat{\mathbb{A}}_{\mu}=\hat{\overline{\mathbb{A}}}_{\mu}=0$. 


\subsection{Gravedad tridimensional no conmutativa}

Representación en términos de tríada y conexión de Ricci

En la sección anterior hemos definido una teoría de gauge no conmutativa, en cuyo límite $\theta^{\mu \nu}=0$ recuperamos la forma de Chern-Simons de la gravitación en tres dimensiones, junto con dos campos de gauge $U(1)$ con acción de Chern-Simons.

Estamos entonces en condiciones de preguntarnos si nuestra teoría puede ser formulada en términos de variables tríada $\hat{e}_{\mu}^{a}$ y conexión de spin $\hat{w}^{a b}{ }_{\mu}$ "no conmutativas". Si aceptamos que la relación entre estas variables y las componentes $S L(2, \mathbb{C})$ de los campos de gauge $\hat{A}_{\mu}^{a}$ y $\hat{\bar{A}}_{\mu}^{a}$ es la misma que en el caso conmutativo, obtenemos una generalización no conmutativa natural, es decir

$$
\begin{aligned}
\hat{e}_{\mu}^{a} & =\frac{l}{2 i}\left(\hat{A}_{\mu}^{a}-\hat{\bar{A}}_{\mu}^{a}\right), \\
\hat{w}_{\mu}^{a} & =\frac{1}{2}\left(\hat{A}_{\mu}^{a}+\hat{\bar{A}}_{\mu}^{a}\right)=-\frac{1}{2} \epsilon^{a b c} w_{\mu}^{b c} .
\end{aligned}
$$

Sumando y restando las ecuaciones de Chern-Simons para $\hat{A}_{\mu}^{a}$ y $\hat{\bar{A}}_{\mu}^{a}$, se prueba directamente que $e_{\mu}^{a}$ y $w_{\mu}^{a}$ satisfacen la "ecuaciones de estructura de Cartan no conmutativas"

$$
\begin{aligned}
& R^{a}{ }_{\mu \nu}=-\frac{i}{2}\left(\left[\hat{\mathbb{A}}_{\mu}+\hat{\overline{\mathbb{A}}}_{\mu}, \hat{w}^{a}{ }_{\nu}\right]+\left[\hat{w}^{a}{ }_{\mu}, \hat{\mathbb{A}}_{\nu}+\hat{\overline{\mathbb{A}}}_{\nu}\right]+\frac{i}{l}\left[\hat{\mathbb{A}}_{\mu}-\hat{\overline{\mathbb{A}}}_{\mu}, e^{a}{ }_{\nu}\right]+\left[e^{a}{ }_{\mu}, \hat{\mathbb{A}}_{\nu}-\hat{\overline{\mathbb{A}}}_{\nu}\right]\right) \\
& T^{a}{ }_{\mu \nu}=-\frac{i}{2}\left(\left[\hat{\mathbb{A}}_{\mu}-\hat{\mathbb{A}}_{\mu}, \hat{w}^{a}{ }_{\nu}\right]+\left[\hat{w}^{a}{ }_{\mu}, \hat{\mathbb{A}}_{\nu}-\hat{\overline{\mathbb{A}}}_{\nu}\right]+\frac{i}{l}\left[\hat{\mathbb{A}}_{\mu}+\hat{\widehat{\mathbb{A}}}_{\mu}, e^{a}{ }_{\nu}\right]+\left[e^{a}{ }_{\mu}, \hat{\mathbb{A}}_{\nu}+\hat{\overline{\mathbb{A}}}_{\nu}\right]\right)
\end{aligned}
$$

mientras que, para el campo $U(1)$ obtenemos

$$
\begin{aligned}
& \hat{\mathbb{F}}_{\mu \nu}=-2 i\left(\left[w_{\mu}^{a}, w^{a}{ }_{\nu}\right]+\frac{1}{l^{2}}\left[e_{\mu}^{a}, e_{\nu}^{a}\right]+\frac{i}{l}\left(\left[e_{\mu}^{a}, w_{\nu}^{a}\right]+\left[w_{\mu}^{a}, e_{\nu}^{a}\right]\right)\right), \\
& \hat{\overline{\mathbb{F}}}_{\mu \nu}=-2 i\left(\left[w_{\mu}^{a}, w^{a}{ }_{\nu}\right]+\frac{1}{l^{2}}\left[e_{\mu}^{a}, e^{a}{ }_{\nu}\right]-\frac{i}{l}\left(\left[e_{\mu}^{a}, w^{a}{ }_{\nu}\right]+\left[w_{\mu}^{a}, e_{\nu}^{a}{ }_{\nu}\right]\right)\right) .
\end{aligned}
$$

En estas ecuaciones, la curvatura de gauge $U(1), \hat{\mathbb{F}}_{\mu \nu}$ esta definida como en (9.6), y los tensores de curvatura y de torsión tienen la forma

$$
\begin{aligned}
R_{\mu \nu}^{a} & =\partial_{\mu} \hat{w}^{a}{ }_{\nu}-\partial_{\nu} \hat{w}_{\mu}^{a}-\frac{1}{2} \epsilon^{a b c}\left\{\hat{w}_{\mu}^{b}, \hat{w}^{c}{ }_{\nu}\right\}+\frac{1}{2 l^{2}} \epsilon^{a b c}\left\{\hat{e}_{\mu}^{b}, \hat{e}_{\nu}^{c}\right\}, \\
T_{\mu \nu}^{a} & =\partial_{\mu} \hat{e}^{a}{ }_{\nu}-\partial_{\nu} \hat{e}^{a}{ }_{\mu}-\frac{1}{2} \epsilon^{a b c}\left(\left\{\hat{e}_{\mu}^{b}, \hat{w}_{\nu}^{c}\right\}+\left\{\hat{w}_{\mu}^{b}, \hat{e}^{c}{ }_{\nu}\right\}\right) .
\end{aligned}
$$

Se observa inmediatamente que, siempre que esté presente el campo $U(1)$, el espacio no conmutativo tiene torsión no nula. Por otro lado, los campos gravitatorios actúan como fuente para el campo $U(1)$, por lo que podemos decir que están cargados frente a ese campo. 
Si se desea simplificar aún más estas ecuaciones, podemos definir el dreivein $\hat{e}_{\mu}=\hat{e}^{a}{ }_{\mu} J_{a}-$ $(l / 2)\left(\hat{\mathbb{A}}_{\mu}-\hat{\mathbb{A}}_{\mu}\right)$ y la conexión de spin $\hat{w}_{\mu}=\hat{w}_{\mu}^{a} J_{a}+(i / 2)\left(\hat{\mathbb{A}}_{\mu}+\hat{\mathbb{A}}_{\mu}\right)$, ambos valuados en el algebra de $G L(2, C)$ (nótese la cuarta componente en la identidad, ausente en el caso conmutativo). Entonces las ecuaciones toman la forma

$$
\begin{aligned}
\partial_{\mu} \hat{w}_{\nu}-\partial_{\nu} \hat{w}_{\mu}+\left[\hat{w}_{\mu}, \hat{w}_{\nu}\right]-\frac{1}{l^{2}}\left[\hat{e}_{\mu}, \hat{e}_{\nu}\right] & =0, \\
\partial_{\mu} \hat{e}_{\nu}-\partial_{\nu} \hat{e}_{\mu}+\left[\hat{w}_{\mu}, \hat{e}_{\nu}\right]-\left[\hat{e}_{\mu}, \hat{w}_{\nu}\right] & =0,
\end{aligned}
$$

que, superficialmente, coinciden con las condiciones usuales de torsion y curvatura para la conexión de spin y el tríada valuados en el álgebra. Sin embargo, no debe olvidarse que en estas ecuaciones están involucrados los campos $U(1)$ que, si bien ocultos por la notación, se acoplan a las variables gravitatorias de manera no trivial como puede verse en (9.5-9.6)

Como un procedimiento alternativo, podemos invertir las relaciones (9.9-9.8) para obtener los campos de gauge y reemplazar en la acción (9.3), con lo que obtenemos como acción para las nuevas variables

$$
S^{\theta}\left[\hat{w}^{a}{ }_{\mu}, \hat{e}^{a}{ }_{\mu}, \hat{\mathbb{A}}_{\mu}, \hat{\mathbb{A}}_{\mu}\right]=S_{E H}^{\theta}\left[\hat{w}^{a}{ }_{\mu}, \hat{e}^{a}{ }_{\mu}\right]+i \hat{S}_{C S}\left[\hat{\mathbb{A}}_{\mu}\right]-i S_{C S}^{\theta}\left[\hat{\mathbb{A}}_{\mu}\right]+S_{I n t}^{\theta}\left[\hat{\mathbb{A}}_{\mu}, \hat{\widehat{A}}_{\mu}, \hat{w}_{\mu}^{a}, \hat{e}^{a}{ }_{\mu}\right],
$$

donde $S_{C S}^{\theta}$ es la acción de Chern-Simons no conmutativa $U(1)$ para los campos $\mathbb{A}_{\mu}$ y $\hat{\mathbb{A}}_{\mu}, S_{E H}^{\theta}$ es la "acción de Einstein-Hilbert no conmutativa"

$$
S_{E H}^{\theta}\left[\hat{e}_{\mu}^{a}, \hat{w}^{a}{ }_{\mu}\right]=\frac{2}{l} \int d^{3} x \epsilon_{\mu \nu \rho}\left(R^{a}{ }_{\mu \nu} * \hat{e}_{\rho}^{a}+\frac{1}{3 l^{2}} \epsilon^{a b c} \hat{e}^{a}{ }_{\mu} * \hat{e}^{b}{ }_{\nu} * \hat{e}^{c}{ }_{\rho}\right),(0.1
$$

y $\hat{S}_{\text {Int }}$ es el término de interacción que viene dado por

$$
S_{\text {Int }}^{\theta}=\int d^{3} x\left(\hat{\mathbb{A}}_{\mu} j_{\mu}+\hat{\mathbb{A}}_{\mu} \bar{j}_{\mu}\right),
$$

donde hemos definido las corrientes $j_{\mu}$ y $\bar{j}_{\mu}$ de acuerdo con

$$
\begin{aligned}
& j_{\mu}=-4 i \epsilon_{\mu \nu \rho}\left(w^{a}{ }_{\nu} w_{\rho}^{a}-\frac{1}{l^{2}} e_{\nu}^{a} e_{\rho}^{a}+\frac{i}{l}\left(\left[e_{\nu}^{a}, w_{\rho}^{a}\right]+\left[w_{\nu}^{a}, e_{\rho}^{a}\right]\right)\right), \\
& \bar{j}_{\mu}=4 i \epsilon_{\mu \nu \rho}\left(w_{\nu}^{a}{ }_{\nu} w_{\rho}^{a}-\frac{1}{l^{2}} e_{\nu}^{a} e_{\rho}^{a}-\frac{i}{l}\left(\left[e_{\nu}^{a}, w_{\rho}^{a}\right]+\left[w_{\nu}^{a}{ }_{\nu}, e_{\rho}^{a}\right]\right)\right) .
\end{aligned}
$$

En términos de las variables valuadas en el algebra, $\hat{w}_{\mu} \mathrm{y} \hat{e}_{\mu}$, que hemos definido anteriormente, el conjunto completo de ecuaciones (9.16-9.17) se puede derivar de la "acción no conmutativa de Einstein-Hilbert",

$$
S^{\theta}\left[\hat{e}_{\mu}, \hat{w}_{\mu}\right]=-\frac{2}{l} \operatorname{Tr} \int d^{3} x \epsilon_{\mu \nu \rho}\left(R_{\mu \nu} * \hat{e}_{\rho}-\frac{2}{3 l^{2}} \hat{e}_{\mu} * \hat{e}_{\nu} * \hat{e}_{\rho}\right),
$$


donde $R_{\mu \nu}=\partial_{\mu} \hat{w}_{\nu}-\partial_{\nu} \hat{w}_{\mu}+\left[\hat{w}_{\mu}, \hat{w}_{\nu}\right]$. La variación con respecto al la tríada $\hat{e}_{\mu}$ origina (9.16), mientras que la variación con respecto a la conexión de spin $\hat{w}_{\mu}$ origina (9.17). La diferenciabilidad de esta acción queda asegurada dadas las condiciones de contorno discutidas anteriormente.

Se debe insistir en que, más allá de la similitud entre (9.23) y la acción de Einstein-Hilbert usual, en la primera los campos abelianos $\hat{\mathbb{A}}_{\mu}$ y $\hat{\mathbb{A}}_{\mu}$ están acoplados a $\hat{e}^{a}{ }_{\mu}$ y $\hat{w}^{a}{ }_{\mu}$ de manera no trivial, siendo los acoplamientos proporcionales a potencias positivas de $\theta^{\mu \nu}$.

Si, a manera de anzätz, elegimos anular los campos abelianos, las ecs. (9.16-9.17) (o sus equivalentes (9.10-9.11)) se transforman en,

$$
\begin{aligned}
\partial_{\mu} \hat{w}^{a}{ }_{\nu}-\partial_{\nu} \hat{w}^{a}{ }_{\mu}-\frac{1}{2} \epsilon^{a b c}\left\{\hat{w}_{\mu}^{b}, \hat{w}^{c}{ }_{\nu}\right\}-\frac{1}{2 l^{2}} \epsilon^{a b c}\left\{\hat{e}_{\mu}^{b}, \hat{e}^{c}{ }_{\nu}\right\} & =0 \\
\partial_{\mu} \hat{e}^{a}{ }_{\nu}-\partial_{\nu} \hat{e}^{a}{ }_{\mu}-\frac{1}{2} \epsilon^{a b c}\left(\left\{e^{b}{ }_{\mu}, w^{c}{ }_{\nu}\right\}+\left\{w^{b}{ }_{\mu}, e^{c}{ }_{\nu}\right\}\right) & =0
\end{aligned}
$$

Teniendo en cuenta a los tensores de torsión y curvatura introducidos en las ecuaciones (9.10-9.11), la primera ecuación se puede entender como una condición de curvatura no conmutativa contante, escrita en términos de conexiones y la segunda ecuación es la análoga a una condición de torsión nula. Esta ecuación no implica, por supuesto, que la conexión afín sea simétrica.

El mismo anzätz aplicado a las ecuaciones (9.12)-(9.13) implica que deben cumplirse las igualdades

$$
\begin{aligned}
{\left[w_{\mu}^{a}, w_{\nu}^{a}\right]+\frac{1}{l^{2}}\left[e_{\mu}^{a}, e^{a}{ }_{\nu}\right] } & =0 \\
{\left[e_{\mu}^{a}, w_{\nu}^{a}\right]+\left[w_{\mu}^{a}, e_{\nu}^{a}\right] } & =0 .
\end{aligned}
$$

Encontraremos más adelante soluciones explícitas que satisfacen esta condición. Estas ecuaciones se satisfacen idénticamente para $\theta^{\mu \nu}=0$.

Representación en términos de métrica:

La ecuaciones (9.24) y (9.25) tienen la misma forma que las ecuaciones de estructura de Cartan, pero donde todos los productos han sido reemplazados por productos estrella. Es entonces natural preguntarse si existe una formulación en términos de métrica para estas ecuaciones, es decir, si podemos encontrar las correspondientes ecuaciones de Einstein.

Supondremos que los vínculos (9.26-9.27) se satisfacen e intentaremos escribir (9.24-9.25) en términos de la métrica y de la conexión afín. (Véase [85]-[90] para otras soluciones de este problema en cuatro dimensiones.) 
Definiremos la métrica y la conexión afín como

$$
\begin{aligned}
\hat{g}_{\mu \nu} & =-\delta_{a b} \hat{e}_{\mu}^{a} * \hat{e}_{\nu}^{b}, \\
\hat{\Gamma}_{\lambda \rho}^{\mu} & =\epsilon^{a b c} \hat{e}_{a}^{\mu} * \hat{w}_{\rho}^{b}{ }^{b} \hat{e}_{\lambda}^{c}+\hat{e}_{a}^{\mu} * \partial_{\rho} \hat{e}_{\lambda}^{a} .
\end{aligned}
$$

En otras palabras, $\hat{g}_{\mu \nu}$ y $\hat{\Gamma}^{\rho}{ }_{\mu \nu}$ representan, como es usual, la métrica y la conexión en una base coordenada. Dado $\hat{e}_{\mu}^{a}$ y $\hat{w}_{\mu}^{a}$, las fórmulas escritas arriba determinan completamente $\hat{g}_{\mu \nu} \mathrm{y} \hat{\Gamma}_{\nu \rho}^{\mu}$. Si $\hat{e}^{a}{ }_{\mu} \mathrm{y} \hat{w}^{a}{ }_{\mu}$ satisfacen las ecuaciones de Chern-Simons, nos gustaría encontrar las ecuaciones diferenciales satisfechas por $\hat{g}_{\mu \nu}$ y $\hat{\Gamma}_{\nu \rho}^{\mu}$.

La definición de la conexión afín se puede motivar en la invarianza de gauge de la acción. Bajo transformaciones de gauge $\hat{g}$ la conexión de spin transforma como $\hat{w}_{\mu}^{a b} \rightarrow \hat{w}_{\mu}^{\prime a b}=$ $\left[\hat{g}^{-1}\right]_{c}^{a} * \hat{w}^{c d}{ }_{\mu} * \hat{g}_{d}^{b}+\left[\hat{g}^{-1}\right]_{c}^{a} * \partial_{\mu} \hat{g}_{b}^{c}$. Si $\hat{w}^{\prime}=\hat{\Gamma}_{\lambda \sigma}^{\rho}$ es la conexión en un base coordenada, relacionada con la base tangente a través de la matriz $\hat{g}_{\mu}^{a}=\hat{e}_{\mu}^{a}$, la nueva conexión deviene (9.29).

La curvatura en una base de coordenadas es

$$
R_{\nu \rho \sigma}^{\mu}=\partial_{\rho} \hat{\Gamma}_{\nu \sigma}^{\mu}-\partial_{\sigma} \hat{\Gamma}_{\nu \rho}^{\mu}+\hat{\Gamma}_{\epsilon \rho}^{\mu} * \hat{\Gamma}_{\nu \sigma}^{\epsilon}-\hat{\Gamma}_{\epsilon \sigma}^{\mu} * \hat{\Gamma}_{\nu \rho}^{\epsilon}
$$

y se relaciona con $R^{a}{ }_{\mu \nu}$ por medio de la formula

$$
R_{\nu \rho \sigma}^{\mu}=\epsilon^{a b c} \hat{e}_{a}^{\mu} * R_{\rho \sigma}^{b} * \hat{e}_{\nu}^{c}
$$

Esto se sigue de reemplazar directamente (9.29) en (9.30), y expresa el hecho de que la curvatura es un tensor. Como $R_{\mu \nu}^{a}$ satisface (9.24), encontramos la "ecuación de Einstein no conmutativa",

$$
R_{\nu \rho \sigma}^{\mu}=-\frac{1}{l^{2}}\left(\delta_{\rho}^{\mu} \hat{g}_{\sigma \nu}-\delta_{\sigma}^{\mu} \hat{g}_{\rho \nu}\right)+\hat{E}_{\nu \rho \sigma}^{\mu}
$$

donde $\hat{g}_{\mu \nu}$ se define en $(9.28), \mathrm{y}$

$$
\hat{E}_{\nu \rho \sigma}^{\mu}=\frac{1}{2 l^{2}} \hat{e}_{a}^{\mu} *\left(\hat{e}_{[\rho}^{a} * \hat{e}_{\sigma]}^{b}-\hat{e}_{[\sigma}^{b} * \hat{e}_{\rho]}^{a}\right) * \hat{e}_{b \nu}
$$

El primer término en (9.32) es la contribución usual de la constante cosmológica a las ecuaciones de Einstein. Recuérdese, sin embargo, que en esta teoría la métrica es no simétrica. El segundo término $\hat{E}_{\nu \rho \sigma}^{\mu}$ es un efecto puramente no conmutativo, dependiendo del conmutador de Moyal de las tríadas, y no se puede expresar solamente en términos de la métrica.

Resumiendo, dados $\hat{e}_{\mu}^{a}$ y $\hat{w}^{a}{ }_{\mu}$ que satisfagan las ecuaciones de Chern-Simons, entonces la métrica (9.28) y la conexión afín (9.29) satisfacen las ecuaciones de Einstein no conmutativas (9.32). Mostraremos mas abajo una familia de soluciones de estas ecuaciones. 


\subsection{Soluciones}

Todas las soluciones consideradas aquí viven en la topología $M_{3}=T^{2} \times \mathbb{R}$. Las coordenadas locales en $T^{2}$ son $z, \bar{z}$ y $\rho \in \mathbb{R}$. Las componentes del campo de gauge son entonces $\hat{A}_{\mu}=\left(\hat{A}_{z}, \hat{A}_{\bar{z}}, \hat{A}_{\rho}\right)$. Tomaremos $\theta^{\rho z}=\theta^{\rho \bar{z}}=0$ mientras que las coordenadas no conmutativas satisfacen,

$$
[z, \bar{z}]=\theta
$$

Se debe notar que esta elección de la componentes de $\theta^{\mu \nu}$ y de la variedad $\mathcal{M}$ nos situa en el caso especial estudiado en el capítulo 7.1, es decir cuando $\theta^{\mu \nu}$ no tiene componentes en las direcciones perpendiculares al borde de la variedad. En tal caso y de acuerdo a lo estudiado en dicho capítulo, basta con anular una de las componentes del potencial vector sobre la superficie para tener una acción diferenciable e invariante de gauge. Elegiremos entonces la condición de contorno $\left.\hat{A}_{\bar{z}}\right|_{\partial \mathcal{M}}=0$ y $\left.\hat{\bar{A}}_{z}\right|_{\partial \mathcal{M}}=0$.

Debería ser claro que el agujero negro en tres dimensiones [37]-[38] es una solución de las ecuaciones completas no conmutativas, simplemente porque este campo tiene dos vectores de Killing $\partial_{z}$ y $\partial_{\bar{z}}$, los cuales efectivamente reducen el producto estrella al producto usual.

Para explorar la estructura no conmutativa, necesitamos buscar soluciones más generales. Comenzaremos buscando soluciones a las ecuaciones no conmutativas de Chern-Simons.

La solución quiral

Reescribiremos la ecuación (9.5) en la forma

$$
\begin{aligned}
& \hat{\mathrm{F}}_{\rho z}[\hat{\mathrm{A}}]+i\left[\hat{\mathbb{A}}_{\rho}, \hat{\mathrm{A}}_{z}\right]+i\left[\hat{\mathrm{A}}_{\rho}, \hat{\mathbb{A}}_{z}\right]=0, \\
& \hat{\mathrm{F}}_{\rho \bar{z}}[\hat{\mathrm{A}}]+i\left[\hat{\mathbb{A}}_{\rho}, \hat{\mathrm{A}}_{\bar{z}}\right]+i\left[\hat{\mathrm{A}}_{\rho}, \hat{\mathbb{A}}_{\bar{z}}\right]=0, \\
& \hat{\mathrm{F}}_{z \bar{z}}[\hat{\mathrm{A}}]+i\left[\hat{\mathbb{A}}_{z}, \hat{\mathrm{A}}_{\bar{z}}\right]+i\left[\hat{\mathrm{A}}_{z}, \hat{\mathbb{A}}_{\bar{z}}\right]=0 .
\end{aligned}
$$

Para resolver estas ecuaciones podemos fijar el gauge a

$$
\hat{\mathrm{A}}_{\rho}=i J_{3}, \quad \hat{\mathbb{A}}_{\rho}=0 .
$$

Las dos primeras ecuaciones de (9.35) se transforman en

$$
\begin{aligned}
& \partial_{\rho} \hat{\mathrm{A}}_{z}+\left[\hat{\mathrm{A}}_{\rho}, \hat{\mathbb{A}}_{z}\right]=0, \\
& \partial_{\rho} \hat{\mathrm{A}}_{\bar{z}}+\left[\hat{\mathrm{A}}_{\rho}, \hat{\mathbb{A}}_{\bar{z}}\right]=0,
\end{aligned}
$$

aquí, dado que $\hat{\mathrm{A}}_{\rho}$ es constante, el conmutador no contiene productos de Moyal. La solución de estas ecuaciones es

$$
\hat{\mathrm{A}}_{z}=d^{-1} \tilde{\mathrm{A}}_{z}(z, \bar{z}) d, \quad \hat{\mathrm{A}}_{\bar{z}}=d^{-1} \tilde{\mathrm{A}}_{\bar{z}}(z, \bar{z}) d,
$$


donde $d=e^{i \rho J^{3}}$ y hemos incluido dos matrices arbitrarias $\tilde{\mathrm{A}}_{z}(z, \bar{z})$ y $\tilde{\mathrm{A}}_{\bar{z}}(z, \bar{z})$ que son funciones de $z, \bar{z}$ pero no de $\rho$. La condición de contorno $\left.\hat{A}_{\bar{z}}\right|_{\partial M}=0$ implica $\tilde{\mathrm{A}}_{\bar{z}}=0$, o sea que $\hat{\mathrm{A}}_{\bar{z}}=0$.

Reescribiendo ahora la ecuación (9.6), tenemos que

$$
\begin{aligned}
& \hat{\mathbb{F}}_{\rho z}-i \operatorname{Tr}\left[\hat{\mathrm{A}}_{\rho}, \hat{\mathrm{A}}_{z}\right]=0, \\
& \hat{\mathbb{F}}_{\rho \bar{z}}-i \operatorname{Tr}\left[\hat{\mathrm{A}}_{\rho}, \hat{\mathrm{A}}_{\bar{z}}\right]=0, \\
& \hat{\mathbb{F}}_{z \bar{z}}-i \operatorname{Tr}\left[\hat{\mathrm{A}}_{z}, \hat{\mathrm{A}}_{\bar{z}}\right]=0 .
\end{aligned}
$$

Usando $\hat{\mathrm{A}}_{\bar{z}}=0$ y la condición de gauge (9.36), las dos primeras ecuaciones en (9.39) se escriben

$$
\partial_{\rho} \hat{\mathbb{A}}_{z}=\partial_{\rho} \widehat{\mathbb{A}}_{\bar{z}}=0,
$$

Se ve entonces que $\hat{\mathbb{A}}_{z}$ y $\widehat{\mathbb{A}}_{\bar{z}}$ deben ser independientes de $\rho$. Dada la condición de contorno $\left.\hat{\mathbb{A}}_{\bar{z}}\right|_{\partial M}=0$, esto implica que $\hat{\mathbb{A}}_{\bar{z}}=0$ en todas partes. La ecuación que resta en (9.39) es

$$
\partial_{\bar{z}} \hat{\mathbb{A}}_{z}=0,
$$

y entonces $\hat{\mathbb{A}}_{z}=\widehat{\mathbb{A}}_{z}(z)$ es una función analítica arbitraria de $z$. Con esta solución para el campo $U(1)$, la última ecuación en (9.35) se simplifica, transformándose en

$$
\partial_{\bar{z}} \hat{\mathrm{A}}_{z}=0
$$

o sea $\hat{\mathrm{A}}_{z}=\hat{\mathrm{A}}_{z}(z)$.

Entonces, la solución general a las ecuaciones (9.5-9.6) con condiciones de contorno $\left.\hat{\mathrm{A}}_{\bar{z}}\right|_{\partial M}=\left.\hat{\mathbb{A}}_{\bar{z}}\right|_{\partial M}=0$, la cual está cercanamente relacionada con el agujero negro en tres dimensiones, es la siguiente solución quiral,

$$
\begin{gathered}
\hat{\mathrm{A}}_{z}=d^{-1} \tilde{\mathrm{A}}_{z}(z) d, \quad \hat{\mathrm{A}}_{\rho}=i J_{3}=d^{-1} \partial_{\rho} d, \quad \hat{\mathbb{A}}_{z}=\hat{\mathbb{A}}_{z}(z), \\
\hat{\mathbb{A}}_{\rho}=\hat{\mathbb{A}}_{\bar{z}}=\hat{\mathrm{A}}_{\bar{z}}=0,
\end{gathered}
$$

donde $\widetilde{\mathrm{A}}_{z}(z)+i \hat{\mathbb{A}}_{z}(z)$ es una función arbitraria de $z$ valuada en el álgebra de Lie de $G L(2, \mathbb{C})$. Esta configuración resuelve tanto las ecuaciones conmutativas cuanto las no conmutativas. Se puede también comprobar que se trata de un punto fijo bajo el mapeo de Seiberg y Witten [19]. Un análisis similar para el segundo campo complejo $\hat{\bar{A}}_{\mu}$ lleva a una solución análoga a (9.43) pero con $\hat{\mathrm{A}}_{z}(z) \rightarrow \hat{\overline{\mathrm{A}}}_{\bar{z}}(\bar{z}), \hat{\mathbb{A}}_{z}(z) \rightarrow \hat{\overline{\mathbb{A}}}_{\bar{z}}(\bar{z})$ y $d \rightarrow d^{-1}$.

Una transformación de gauge (con elemento del grupo $d$ ) lleva la solución a la forma más simple

$$
\begin{gathered}
\hat{\mathrm{A}}_{z}=\tilde{\mathrm{A}}_{z}(z), \quad \hat{\mathbb{A}}_{z}=\hat{\mathbb{A}}_{z}(z), \\
\hat{\mathrm{A}}_{\bar{z}}=\hat{\mathrm{A}}_{\rho}=\hat{\mathbb{A}}_{\bar{z}}=\hat{\mathbb{A}}_{\rho}=0 .
\end{gathered}
$$


Una propiedad importante de (9.44) es la simetría de Kac-Moody bajo transformaciones de gauge holomorfas. Para ver esto, especialicemos al caso $\hat{\mathbb{A}}_{z}=0$ y notemos que la configuración (9.44) es invariante de forma bajo transformaciones de gauge que sólo dependen de $z$. Hagamos $\hat{\lambda}=\hat{\lambda}(z)$. Actuando con la transformación no conmutativa (4.10) se encuentra que,

$$
\begin{aligned}
& \delta \hat{A}_{z}=\partial_{z} \hat{\lambda}+\hat{A}_{z} * \hat{\lambda}-\hat{\lambda} * \hat{A}_{z}=\partial_{z} \hat{\lambda}+\hat{A}_{z} \hat{\lambda}-\hat{\lambda} \hat{A}_{z} \\
& \delta \hat{A}_{\bar{z}}=0 \\
& \delta \hat{A}_{\rho}=0
\end{aligned}
$$

El producto estrella ha sido eliminado porque la solución sólo depende de $z$. Esta simetría del espacio de soluciones (9.44) es generada por un álgebra de Kac-Moody y juega un rol importante en la comprensión de la entropía del agujero negro en tres dimensiones.

\section{La métrica}

En esta sección construiremos la métrica correspondiente a la solución quiral de la sección anterior. Utilizaremos la ecuación (9.28) donde la tríada $\hat{e}^{a}{ }_{\mu}$ estará construida de acuerdo a (9.8).

Utilizando la solución quiral (9.43) en la definición de la tríada (9.8), nos queda

$$
\hat{e}_{z}^{a} J_{a}=\frac{l}{2 i} d^{-1} \tilde{\mathrm{A}}_{z}(z) d, \quad \hat{e}_{\bar{z}}^{a} J_{a}=-\frac{l}{2 i} d \tilde{\overline{\mathrm{A}}}_{\bar{z}}(\bar{z}) d^{-1},
$$

Definimos las componentes de las matrices $S L(2, \mathbb{C}), \tilde{\mathrm{A}}_{z}$ y $\tilde{\mathrm{A}}_{\bar{z}}$ de acuerdo con

$$
\tilde{\mathrm{A}}_{z}=\frac{i}{2}\left(\begin{array}{cc}
A^{3} & A^{+} \\
A^{-} & -A^{3}
\end{array}\right), \quad \tilde{\overline{\mathrm{A}}}_{\bar{z}}=\frac{i}{2}\left(\begin{array}{cc}
\bar{A}^{3} & \bar{A}^{+} \\
\bar{A}^{-} & -\bar{A}^{3}
\end{array}\right) .
$$

Entonces la parte simétrica de la métrica, asociada a la longitud de arco $d s^{2}=g_{\mu \nu} d x^{\mu} d x^{\nu}$, es

$$
\begin{aligned}
d s^{2}= & l^{2} d \rho^{2}-\frac{l^{2}}{4}\left(A^{3^{2}}+A^{+} A^{-}\right) d z^{2}-\frac{l^{2}}{4}\left(\bar{A}^{3^{2}}+\bar{A}^{+} \bar{A}^{-}\right) d \bar{z}^{2} \\
& +\frac{l^{2}}{8}\left(2\left\{A^{3}, \bar{A}^{3}\right\}+\left\{A^{-}, \bar{A}^{+}\right\} e^{-2 \rho}+\left\{A^{+}, \bar{A}^{-}\right\} e^{2 \rho}\right) d z d \bar{z} \\
& +i l^{2} \bar{A}^{3} d \bar{z} d \rho-i l^{2} A^{3} d z d \rho .
\end{aligned}
$$

En este punto, estamos interesados en determinar las condiciones que deben ser impuestas a los campos de gauge para obtener una métrica que sea asintóticamente anti de Sitter. Con este fin, seguiremos el método de la ref. [40] extendido al caso no conmutativo. Las componentes no diagonales que contienen $\rho$ deben ser nulas. Esto se puede asegurar tomando 
$A^{3}=\bar{A}^{3}=0$, lo que extiende al caso no conmutativo la primera condición de reducción de Polyakov. La métrica resultante es

$$
d s^{2}=l^{2} d \rho^{2}-\frac{l^{2}}{4} A^{+} A^{-} d z^{2}-\frac{l^{2}}{4} \bar{A}^{+} \bar{A}^{-} d \bar{z}^{2}+\frac{l^{2}}{8}\left(\left\{\bar{A}^{+}, A^{-}\right\} e^{-2 \rho}+\left\{A^{+}, \bar{A}^{-}\right\} e^{2 \rho}\right) d z d \bar{z},
$$

la cual tiene como forma asintótica $(\rho \rightarrow \infty)$

$$
d s^{2}=l^{2} d \rho^{2}+\frac{l^{2}}{8}\left\{A^{+}, \bar{A}^{-}\right\} e^{2 \rho} d z d \bar{z} .
$$

Entonces, para tener una forma AdS es necesario imponer

$$
\left\{A^{+}, \bar{A}^{-}\right\}=8 \text {. }
$$

Derivando respecto de $z$ y $\bar{z}$ obtenemos las siguientes relaciones (recuérdese que $A^{+}$es holomorfa y $\bar{A}^{-}$es antiholomorfa)

$$
\left\{\partial_{z} A^{+}, \bar{A}^{-}\right\}=0, \quad\left\{A^{+}, \partial_{\bar{z}} \bar{A}^{-}\right\}=0
$$

En el caso usual conmutativo, estas relaciones implican $A^{+}, \bar{A}^{-}$constantes. Para comprobar esto en el caso no conmutativo, obsérvese que, siguiendo [83], se puede escribir

$$
\begin{aligned}
& \hat{f}(z) * \hat{g}(\bar{z})=e^{\frac{\theta}{2} \partial \bar{\partial}} \hat{f}(z) \hat{g}(\bar{z}), \\
& \hat{g}(\bar{z}) * \hat{f}(z)=e^{-\frac{\theta}{2} \partial \bar{\partial}} \hat{f}(z) \hat{g}(\bar{z}) \text {, }
\end{aligned}
$$

lo cual implica

$$
\begin{aligned}
\frac{1}{2}\{\hat{f}, \hat{g}\} & =\frac{1}{2}\left(e^{\frac{\theta}{2} \partial \bar{\partial}}+e^{-\frac{\theta}{2} \partial \bar{\partial}}\right) \hat{f}(z) \hat{g}(\bar{z})= \\
& =\cosh \left(\frac{\theta}{2} \partial \bar{\partial}\right) \hat{f}(z) \hat{g}(\bar{z}) .
\end{aligned}
$$

Usando esto, la ecuación (9.54) se puede reescribir como

$$
\cosh \left(\frac{\theta}{2} \partial \bar{\partial}\right)\left(\partial_{z} A^{+} \bar{A}^{-}\right)=0, \quad \cosh \left(\frac{\theta}{2} \partial \bar{\partial}\right)\left(A^{+} \partial_{\bar{z}} \bar{A}^{-}\right)=0 .
$$

Llamando $\left\{\psi_{\lambda}\right\}$ a un conjunto completo de autofunciones del laplaciano $\partial \bar{\partial}$ con autovalores $\{\lambda\}$, se puede escribir $\cosh ((\theta / 2) \partial \bar{\partial})=\sum_{\lambda} \cosh ((\theta / 2) \lambda)\left|\psi_{\lambda}\right\rangle\left\langle\psi_{\lambda}\right|$. Esto asegura que $\cosh (\theta / 2 \partial \bar{\partial})$ no tiene modos cero, y entonces de (9.57) se puede deducir

$$
\partial_{z} A^{+} \bar{A}^{-}=0, \quad A^{+} \partial_{\bar{z}} \bar{A}^{-}=0,
$$


esto implica que $A^{+}, \bar{A}^{-}$deben ser constantes. Hemos encontrado entonces la segunda condición de reducción, que escribiremos como $A^{+}=2, \bar{A}^{-}=2$.

Concluimos que para tener una métrica asintóticamente AdS en el caso no conmutativo, es necesario imponer sólo las condiciones usuales de reducción de Polyakov, discutidas en [40]. En este caso, la solución quiral (9.43) toma la forma

$$
\begin{gathered}
\hat{\mathrm{A}}_{z}=i\left(\begin{array}{ll}
0 & e^{\rho} \\
\frac{1}{2 l} T(z) e^{-\rho} & 0
\end{array}\right), \quad \hat{\mathrm{A}}_{\rho}=-\overline{\mathrm{A}}_{\rho}=i J_{3}, \quad \hat{\overline{\mathrm{A}}}_{\bar{z}}=i\left(\begin{array}{cc}
0 & \frac{1}{2 l} \bar{T}(\bar{z}) e^{-\rho} \\
e^{\rho} & 0
\end{array}\right), \\
\hat{\mathbb{A}}_{z}=\hat{\mathbb{A}}_{z}(z) \quad \hat{\overline{\mathbb{A}}}_{\bar{z}}=\hat{\overline{\mathbb{A}}}_{\bar{z}}(\bar{z}) \\
\hat{\mathrm{A}}_{\bar{z}}=\hat{\overline{\mathrm{A}}}_{z}=\hat{\mathbb{A}}_{\bar{z}}=\hat{\mathbb{A}}_{\rho}=\hat{\overline{\mathbb{A}}}_{z}=\hat{\overline{\mathbb{A}}}_{\rho}=0 .
\end{gathered}
$$

Con esto, la parte simétrica de la métrica, como se definió en (9.50), deviene

$$
d s^{2}=l^{2} d \rho^{2}-\frac{l}{2} T d z^{2}-\frac{l}{2} \bar{T} d \bar{z}^{2}+\frac{1}{8}\left(\{\bar{T}, T\} e^{-2 \rho}+8 l^{2} e^{2 \rho}\right) d z d \bar{z} .
$$

Vemos que la única componente de la parte simétrica de la métrica afectada por la no conmutatividad es $\hat{g}_{z \bar{z}}^{S}=(1 / 8)\{\bar{T}, T\} \exp (-2 \rho)$. Usando (9.55) para reescribir el anticonmutador, esta componente se puede reescribir como

$$
\hat{g}_{z \bar{z}}^{S}=\cosh \left(\frac{\theta}{2} \partial \bar{\partial}\right) g_{z \bar{z}}
$$

siendo $g_{\mu \nu}$ la métrica construída en [39] para el caso conmutativo. El operador $\cosh ((\theta / 2) \partial \bar{\partial})$ actúa como la identidad cuando se aplica a las otras componentes de la métrica, dado que todas las derivadas se anulan,

$$
\hat{g}_{z z}^{S}=\cosh \left(\frac{\theta}{2} \partial \bar{\partial}\right) g_{z z}, \quad \hat{g}_{\bar{z} \bar{z}}^{S}=\cosh \left(\frac{\theta}{2} \partial \bar{\partial}\right) g_{\bar{z} \bar{z}}
$$

Entonces, la relación entre la solución usual conmutativa y la parte simétrica de la solución no conmutativa se puede escribir en la forma compacta

$$
\hat{g}_{\mu \nu}^{S}=\cosh \left(\frac{\theta}{2} \partial \bar{\partial}\right) g_{\mu \nu} .
$$

La métrica completa $\hat{g}_{\mu \nu}=\hat{g}_{\mu \nu}^{S}+\hat{g}_{\mu \nu}^{A}$, donde $\hat{g}_{\mu \nu}^{A}$ es la parte antisimétrica, satisface las ecuaciones de Einstein no conmutativas (9.32). Nótese que $\hat{g}_{\mu \nu}^{A}$ es de hecho no nula. Esta contribución no nula proviene de

$$
\hat{g}_{z \bar{z}}=\exp \left(\frac{\theta}{2} \partial \bar{\partial}\right) g_{z \bar{z}}, \quad \hat{g}_{\bar{z} z}=\exp \left(-\frac{\theta}{2} \partial \bar{\partial}\right) g_{\bar{z} z}
$$

lo cual implica

$$
\hat{g}_{z \bar{z}}^{A}=\sinh \left(\frac{\theta}{2} \partial \bar{\partial}\right) g_{z \bar{z}}
$$


Recuérdese que la derivación de (9.32) a partir de las ecuaciones de Einstein ordinarias esta codificada en la combinación $\hat{E}_{\nu \rho \sigma}^{\mu}$ la cual depende del conmutador $\left[\hat{e}_{\rho}^{a}, \hat{e}_{\sigma}^{b}\right]$. En el presente caso, la única contribución no nula a este conmutador es la componente $(\rho=z, \sigma=\bar{z})$, que es proporcional a $[T, \bar{T}]=2 \sinh \left(\frac{\theta}{2} \partial \bar{\partial}\right) T(z) \bar{T}(\bar{z})$.

\subsection{Alcances y limitaciones de nuestra teoría}

Como los éxitos y puntos abiertos de este capítulo, mencionaremos

- La formulación en términos de métrica de nuestra teoría de la gravitación no conmutativa, conduce a ecuaciones de Einstein que contienen un término adicional que involucra al tensor $E_{\nu \rho \sigma}^{\mu}$, cuya definición contiene las tríadas $\hat{e}_{\mu}^{a}$. Para que las ecuaciones de Einstein queden escritas en forma completamente independiente de estas variables, sería de interés encontrar algún tipo de interpretación geométrica para este tensor.

- Existen en la literatura varias formulaciones de la gravitación no conmutativa [9]-[14], [85]-[90]. Es importante investigar la relación que estas teorías puedan tener con la definición aquí propuesta.

- En otras formulaciones de la gravitación no conmutativa [87]-[90], el determinante la métrica que aparece en el elemento invariante de volumen en la acción de Einstein, presenta problemas de ordenamiento en su desarrollo en serie de potencias. Con nuestra definición de la gravitación no conmutativa, hemos sorteado estos problemas al utilizar la acción de Chern-Simons para definir la dinámica.

- En este capítulo nos hemos centrado en el caso de la gravitación euclídea y con constante cosmológica negativa. La extensión a otros valores de la constante cosmológica o a la signatura minkowskiana parece directa. En cada caso, será necesario ampliar el grupo de gauge que su álgebra de Lie cierre por anticonmutacion

- Se puede objetar que la inclusión de los campos $U(1)$ asociados al generador adicional resulta antinatural. Es ese caso, sería necesario adaptar los resultados de [63]-[65] a la teoría de Chern-Simons, para poder formular una teoría de la gravitación no conmutativa sin generadores adicionales

\subsection{Conclusiones}

Resumiremos aquí nuestras conclusiones

- Hemos formulado una teoría de Chern-Simons no conmutativa, con grupo de gauge $G L(2, \mathbb{R})$. El límite conmutativo de esta teoría coincide, a nivel clásico, con la forma de 
Chern-Simons de la gravitación en $d=3$ dimensiones euclídeas, sumada a un campo de gauge complejo $U(1)$, desacoplado y con acción de Chern-Simons.

Para $\theta^{\mu \nu}$ finito, si bien el acoplamiento entre el campo $U(1)$ y la parte $S L(2, \mathbb{R})$ es no trivial, hemos propuesto considerar esta teoría como la forma de Chern-Simons de una teoría de gravitación no conmutativa en $d=3$ dimensiones euclídeas.

- Hemos sido capaces de reformular dicha teoría de Chern-Simons en términos de un dreibein y conexión de spin no conmutativos, cuyas ecuaciones de movimiento generalizan las ecuaciones de Einstein en este formalismo.

Verificamos que es posible definir una acción de Einstein escrita en términos de los campos no conmutativos, de la cual se derivan las correctas ecuaciones de movimiento, sin necesidad de definir algún ordenamiento para el determinante del dreibein, sorteando de esta manera las dificultades que se han discutido en la literatura [87]-[90].

- Encontramos soluciones a nuestras ecuaciones que se corresponden en el límite conmutativo a las soluciones conocidas de la teoría de gravitación en 3 dimensiones, por ejemplo la solución afín y la solución de agujero negro BTZ.

Además, hemos encontrado un operador que mapea las soluciones de nuestra teoría no conmutativa el las correspondientes soluciones de la teoría conmutativa. 


\section{Parte III}

\section{Teoría de Born-Infeld en espacio no conmutativo}



En esta última parte de la tesis, estudiaremos algunos aspectos de la teoría de Born-Infeld en espacio no conmutativo.

Primeramente, resumiremos las características principales de la acción de Born-Infeld conmutativa. Repasaremos su definición para grupo de gauge abeliano y no abeliano, los problemas de ordenamiento que aparecen en este último caso, y su relación con la supersimetría. Luego definiremos una acción de Born-Infeld invariante de gauge en espacio no conmutativo, utilizando para esto una generalización de la prescripción de ordenamiento simétrico que se ha propuesto para el caso no abeliano (capítulo 10).

Hecho esto, estudiaremos la extensión supersimétrica del modelo de Born-Infeld no conmutativo, utilizando para esto una adaptación adecuada de la técnica de supercampos. Con esta acción supersimétrica, estudiaremos la estructura de las ecuaciones de Bogomol'nyi de esta teoría (capítulo 11).

Las contribuciones originales de esta sección de la tesis, fueron desarrolladas en colaboración con F.A. Schaposnik y R.L. Pakman (de la Universidad Nacional de La Plata). 


\title{
Capítulo 10
}

\section{Introducción a la teoría de Born-Infeld}

\begin{abstract}
En esta sección recorreremos algunas propiedades de la acción de Born-Infeld en espacio conmutativo. Comenzaremos dando su definición en el caso abeliano así como su conexión con la forma determinantal Dirac. Luego analizaremos la definición de la acción de Born-Infeld en el caso de una teoría de gauge no abeliana, deteniéndonos un poco en la forma simétrica propuesta por Tseytlin, escribiéndola de una forma ligeramente diferente de la usual. En la última sección de este capítulo propondremos una definición de la acción de Born-Infeld en espacio no conmutativo.
\end{abstract}

\subsection{La acción de Born-Infeld conmutativa}

\section{Introducción}

En 1934 Max Born [91] luego acompañado por Leopold Infeld [92]-[94], desarrolló una teoría general de la electrodinámica no lineal, como alternativa a la teoría de Maxwell. Su principal motivación fue construir una teoría con soluciones clásicas representando partículas eléctricamente cargadas, como los electrones, con autoenergía finita.

Si bien la teoría inicialmente causó interés en físicos como Schrödinger [95], Einstein y Rosen, etc, entró en un período de hibernación por aproximadamente 40 años, asomando en la superficie de vez en cuando. Hace aproximadamente diez años reapareció en conexión con la teoría de cuerdas y modelos sigma [44]-[52].

Más recientemente ha habido una explosión de artículos donde la acción de Born-Infeld juega algún rol en diferentes contextos, en particular, será importantes para lo que sigue los citados en [97]-[101]. 


\section{La acción de Born-Infeld para el caso abeliano}

La acción de Born-Infeld puede escribirse en dos formas equivalentes. En el espacio de Minkowsky en $3+1$ dimensiones se tiene

$$
S_{B I}=-\beta^{2} \int d^{4} x\left(\sqrt{1+\frac{1}{2 \beta^{2}} F_{\mu \nu} F^{\mu \nu}-\frac{1}{16 \beta^{4}}\left(F^{\mu \nu} \tilde{F}_{\mu \nu}\right)^{2}}-1\right),
$$

o bien

$$
S_{B I}=\int d^{4} x\left(\sqrt{-\beta \operatorname{det} g_{\mu \nu}}-\sqrt{-\operatorname{det}\left(\beta g_{\mu \nu}-F_{\mu \nu}\right)}\right) .
$$

debido a su similitud con la acción de Dirac para el modelo extensible del electron [96], esta última forma en determinantes se suele llamar forma de Dirac de la acción de Born-Infeld

Las dimensiones de $\beta$ y $F_{\mu \nu}$ son $[\beta]=\left[F_{\mu \nu}\right]=m^{2}$. La identidad entre ambas formas de $S_{B I}$ se puede probar fácilmente calculando el determinante que aparece bajo la raíz cuadrada en la forma (10.2)

$$
\begin{aligned}
& \left|\begin{array}{llll}
\beta & -F_{01} & -F_{02} & -F_{03} \\
F_{01} & -\beta & -F_{12} & -F_{13} \\
F_{02} & -F_{12} & -\beta & F_{23} \\
F_{03} & -F_{13} & F_{23} & -\beta
\end{array}\right|= \\
= & \beta\left(\beta^{3}-F_{12} F_{23} F_{13}-F_{13} F_{12} F_{23}+\beta F_{13}^{2}+\beta F_{23}^{2}+\beta F_{12}^{2}\right)+\ldots= \\
= & \beta^{4}+(0) \beta^{3}-\left(\frac{1}{2} F_{\mu \nu} F^{\mu \nu}\right) \beta^{2}+(0) \beta+\frac{1}{16}\left(F^{\mu \nu} \tilde{F}_{\mu \nu}\right)^{2} .
\end{aligned}
$$

Se puede verificar que la teoría no lineal coincide con la teoría de Maxwel para valores pequeños de $E$ y $B$. En otras palabras, en el límite $\beta \rightarrow \infty$, tenemos que $S_{B I} \rightarrow S_{\text {Maxwell }}{ }^{1}$.

Como teoría cuántica de campos, el modelo de Born-Infeld no es renormalizable: cuando se expande la raíz cuadrada, aparecen potencias crecientes de $1 / \beta^{2} \sim 1 / m^{4}$. Por supuesto, se puede considerar el lagrangiano de Born-Infeld como el lagrangiano efectivo a baja energía resultante de la integración de modos masivos en algún lagrangiano renormalizable. En el régimen de baja energía, donde se usa el lagrangiano de Born-Infeld, hay un cutoff natural, $\Lambda=\sqrt{\beta}$, el cual puede ser usado para tener resultados perturbativos finitos.

Las ecuaciones de Euler-Lagrange en la teoría de Born-Infeld son

$$
\begin{gathered}
\partial_{\mu}\left(\frac{1}{R}\left(F^{\mu \nu}-\frac{1}{4 \beta^{2}}\left(F^{\alpha \beta} \tilde{F}_{\alpha \beta}\right) \tilde{F}^{\mu \nu}\right)\right)=0 \\
R=\sqrt{1+\frac{1}{2 \beta^{2}} F_{\mu \nu} F^{\mu \nu}-\frac{1}{16 \beta^{4}}\left(F_{\mu \nu} \tilde{F}^{\mu \nu}\right)^{2}} .
\end{gathered}
$$

\footnotetext{
${ }^{1}$ Se puede ver que este requerimieto, unido a la simetría de dualidad, impone varios vínculos sobre la forma de cualquier lagrangiano no lineal. [97]
} 
Si se agrega como fuente en estas ecuaciones una carga eléctrica puntual en el origen, y se considera el caso puramente eléctrico $F^{0 i}=E^{i}, F^{i j}=0$, se tiene como solución

$$
\vec{E}=\frac{e}{4 \pi} \frac{1}{\sqrt{r^{4}+r_{0}^{4}}} \check{r}
$$

con $r_{0}^{2}=e /(4 \sqrt{2} \pi \beta)$. Vemos entonces que el campo eléctrico no diverge en el origen, sino que toma un valor máximo, $E(0)=\sqrt{2} \beta$. Esa es la razón por la cual Born llamó a $\beta$ el "campo absoluto". Las soluciones de las ecuaciones de Born-Infeld cuando son incorporadas fuentes fueron llamadas BIones por Gibbons [52].

La energía total de esta solución se puede hallar a partir del tensor de energía impulso derivado de la acción (10.1). Después de un poco de cálculo se encuentra una respuesta finita $U_{\text {tot }}=1,236 e^{2} / 4 \pi r_{0}$, lo que se podía esperar siendo $E(0)$ finito.

\section{Teorías de Born-Infeld no abelianas}

En la literatura [44]-[48], han sido discutidas varias posibilidades para extender la acción de Born-Infeld abeliana al caso de una simetría de gauge no abeliana. Básicamente, estas difieren en la forma en que la operación de traza sobre el álgebra está definida.

Se puede construir una extensión natural de la propuesta de Yang y Mills a una acción de Born-Infeld, tomando la traza fuera de la raíz cuadrada, es decir

$$
\begin{aligned}
S_{B I} & =-\beta^{2} \operatorname{Tr} \int d^{4} x\left(\sqrt{1+\frac{1}{2 \beta^{2}} F_{\mu \nu} F^{\mu \nu}-\frac{1}{16 \beta^{4}}\left(F^{\mu \nu} \tilde{F}_{\mu \nu}\right)^{2}}-1\right)= \\
& =\operatorname{Tr} \int d^{4} x\left(-\frac{1}{4} F_{\mu \nu} F^{\mu \nu}+\frac{1}{32 \beta^{2}}\left(F^{\mu \nu} \tilde{F}_{\mu \nu}\right)^{2}+\frac{1}{8}\left(\frac{1}{2 \beta} F_{\mu \nu} F^{\mu \nu}-\frac{1}{16 \beta^{3}}\left(F^{\mu \nu} \tilde{F}_{\mu \nu}\right)^{2}\right)^{2}+\cdots\right),
\end{aligned}
$$

donde en la segunda línea hemos desarrollado la raíz cuadrada en serie de potencias para luego tomar la traza sobre los productos de matrices resultantes.

Sin embargo, en el contexto de teoría de cuerdas, una operación de "traza simétrica" como la propuesta por Tseytlin [44] parece ser la apropiada. Una forma de definir esta acción es

$$
\begin{aligned}
S_{B I} & =-\beta^{2} \operatorname{Tr} \mathcal{S} \int d^{4} x\left(\sqrt{1+\frac{1}{2 \beta^{2}} F_{\mu \nu}, F^{\mu \nu}-\frac{1}{16 \beta^{4}}\left(F^{\mu \nu}, \tilde{F}_{\mu \nu}\right)^{2}}-1\right)= \\
& =\operatorname{Tr} \mathcal{S} \int d^{4} x\left(-\frac{1}{4} F_{\mu \nu}, F^{\mu \nu}+\frac{1}{32 \beta^{2}}\left(F^{\mu \nu}, \tilde{F}_{\mu \nu}\right)^{2}+\frac{1}{8}\left(\frac{1}{2 \beta} F_{\mu \nu}, F^{\mu \nu}-\frac{1}{16 \beta^{3}}\left(F^{\mu \nu}, \tilde{F}_{\mu \nu}\right)^{2}\right)^{2}+\cdots\right) .
\end{aligned}
$$

Aquí la operación $\mathcal{S}$, que actúa linealmente en el desarrollo en serie de potencias de la segunda línea, se define sobre un producto arbitrario de componentes de la curvatura de la siguiente 
manera

$$
\mathcal{S}\left(F_{\mu_{1} \nu_{1}}, F_{\mu_{2} \nu_{2}}, \ldots, F_{\mu_{N} \nu_{N}}\right)=\frac{1}{N !} \sum_{\{\sigma\}} F_{\mu_{\sigma_{1}} \nu_{\sigma_{1}}} F_{\mu_{\sigma_{2}} \nu_{\sigma_{2}}} \ldots F_{\mu_{\sigma_{N}} \nu_{\sigma_{N}}}
$$

donde la suma se toma sobre todas las permutaciones de los factores presentes en el miembro derecho.

Es decir que para definir el lagrangiano de Born-Infeld no abeliano, en cada término de la serie de potencias, sumamos sobre los productos ordenados de todas las maneras posibles ("simetrizando" la expresión mediante la operación $\mathcal{S}$ ) y luego tomamos la traza de la serie resultante. El resultado se puede resumar sólo para algunas configuraciones particulares del campo $F_{\mu \nu}[98]$.

Si escribimos $F_{\mu \nu}^{a} t^{a}$, podemos reescribir la traza de la expresión anterior de la siguiente manera

$$
\begin{aligned}
\operatorname{Tr} \mathcal{S}\left(F_{\mu_{1} \nu_{1}}, F_{\mu_{2} \nu_{2}}, \ldots, F_{\mu_{N} \nu_{N}}\right) & =F_{\mu_{1} \nu_{1}}^{a_{1}} F_{\mu_{2} \nu_{2}}^{a_{2}} \ldots F_{\mu_{N} \nu_{N}}^{a_{2}} \frac{1}{N !} \sum_{\{\sigma\}} \operatorname{Tr}\left(t^{a_{\sigma_{1}}} t^{a_{\sigma_{2}}} \ldots t^{a_{\sigma_{N}}}\right)= \\
& =F_{\mu_{1} \nu_{1}}^{a_{1}} F_{\mu_{2} \nu_{2}}^{a_{2}} \ldots F_{\mu_{N} \nu_{N}}^{a_{2}} \operatorname{STr}\left(t^{a_{1}}, t^{a_{2}}, \ldots, t^{a_{N}}\right) .
\end{aligned}
$$

Entonces la operación compuesta $\operatorname{Tr} \mathcal{S}$ se reduce a la prescripción de ordenamiento simétrico para los generadores en el desarrollo en serie de potencias de la raíz cuadrada, o traza simétrica, STr, la cual afecta al producto de $N$ generadores de la siguiente manera

$$
\mathrm{S} \operatorname{Tr}\left(t^{a_{1}}, t^{a_{2}}, \ldots, t^{a_{N}}\right)=\frac{1}{N !} \sum_{\{\sigma\}} \operatorname{Tr}\left(t^{a_{\sigma_{1}}} t^{a_{\sigma_{2}}} \ldots t^{a_{\sigma_{N}}}\right),
$$

con la cual la acción (10.14) se puede reescribir

$$
S_{B I}=-\beta^{2} \operatorname{STr} \int d^{4} x\left(\sqrt{1+\frac{1}{2 \beta^{2}} F_{\mu \nu}, F^{\mu \nu}-\frac{1}{16 \beta^{4}}\left(F^{\mu \nu}, \tilde{F}_{\mu \nu}\right)^{2}}-1\right) .
$$

Nótese que la prescripción (10.11) se debe aplicar exclusivamente sobre los generadores presentes en la curvatura $F_{\mu \nu}^{a} t^{a}$ y no sobre aquéllos presentes en la conexión $A_{\mu}^{a} t^{a}$. Si hiciéramos lo último, al simetrizar desaparecerían aquellos términos en $F_{\mu \nu}$ que contienen conmutadores de $A_{\mu}$.

Sólo cuando se utiliza esta simetrización puede relacionarse el lagrangiano con una expresión en términos de un determinante, ya que desaparecen la ambigüedades relacionadas con el orden del producto de los elementos del determinante

$$
\begin{aligned}
S_{B I} & =-\beta^{2} \operatorname{STr} \int d^{4} x\left(\sqrt{1+\frac{1}{2 \beta^{2}} F_{\mu \nu}, F^{\mu \nu}-\frac{1}{16 \beta^{4}}\left(F^{\mu \nu}, \tilde{F}_{\mu \nu}\right)^{2}}-1\right)= \\
& =\operatorname{STr} \int d^{4} x\left(\sqrt{-\beta \operatorname{det} g_{\mu \nu}}-\sqrt{-\operatorname{det}\left(\beta g_{\mu \nu}-F_{\mu \nu}\right)}\right)
\end{aligned}
$$


que hace contacto con la definición de Dirac para el caso abeliano y es mucho más cercana a las acciones efectivas de branas en las que $g_{\mu \nu}$ es una métrica inducida

Esta definición para la acción de Born-Infeld es la única que lleva a una acción que es linealizada por ecuaciones de Bogomol'nyi y a ecuaciones de movimiento que coinciden con las que surgen de imponer la nulidad de la función $\beta$ para los campos subyacentes en la teoría de supercuerdas abiertas.

Se debe mencionar que aún hay algunos problemas no resueltos concernientes al uso de la traza simétrica en teorías de Born-Infeld no abelianas. En particular, algunas discrepancias entre los resultados provenientes de una teoría de Born-Infeld simetrizada y el espectro esperado en las teorías de branas como se señala en [48].

\section{Supersimetría}

Ya en los 80, interesantes propiedades de la teoría de Born-Infeld en conexión con supersimetría fueron descubiertas por diferentes autores:

Deser y Puzalowski [99] estudiaron el conjunto infinito de acciones no masivas de spin 1 formadas con los dos invariantes algebraicos $F_{\mu \nu} F^{\mu \nu}$ y $F^{\mu \nu} \tilde{F}_{\mu \nu}$. La teoría de Born-Infeld fue una de las teorías supersimetrizables mas sencillas seleccionadas. Estos autores también mostraron que el lagrangiano efectivo de Euler-Heisenberg, que surge de la integración de escalares masivos y fermiones en una teoría supersimétrica coincide sólo hasta el orden sexto en potencias del campo vectorial. A órdenes más altos no hay más parámetros para acomodar de manera de hacer coincidir los dos modelos.

Mas recientemente, la supersimetrización de la acción de Born-Infeld ha sido estudiada en [100]-[101]. La teoría no abeliana supersimétrica ha sido estudiada en [53], donde se ha demostrado que la elección del orden simétrico de los generadores, en la expansión en potencias de la raíz cuadrada, es consistente con la supersimetría.

\subsection{La acción de Born-Infeld no conmutativa}

Llegados a este punto, podemos plantearnos cómo definir la acción de Born-Infeld en espacio no conmutativo.

En el espacio ordinario conmutativo, cuando son necesarias potencias más altas de combinaciones de $F_{\mu \nu}$ y $\tilde{F}_{\mu \nu}$ para generar lagrangianos del tipo del de Born-Infeld, aparece un problema de ordenamiento relacionado con los generadores $t^{a}$, como hemos visto en la sección previa. En el presente caso no conmutativo y no abeliano, nos enfrentamos a un segundo problema de orden relacionado con el producto estrella. Como veremos, ambos problemas se entrelazan y deben ser resueltos ambos a la vez ${ }^{2}$.

\footnotetext{
${ }^{2} \mathrm{Si}$ el interés en la teoría de Born-Infeld viene de la dinámica de $D$-branas, en la cual es relevante cuando se pueden despreciar los términos que contienen derivadas de la curvatura, el problema de ordenamiento puede en principio ser ignorado [19]. No obstante, la equivalencia entre la teoría de gauge no conmutativa y
} 
Si pretendemos aplicar la receta que hemos desarrollado en capítulos anteriores, que nos dice cómo definir la acción de una teoría no conmutativa a partir de su análoga conmutativa, debemos primero escribir la acción conmutativa en términos de matrices del álgebra de Lie $A_{\mu}$, evitando cualquier forma en componentes $A_{\mu}^{a}$. Por lo tanto, aplicaremos nuestra receta de reemplazar campos conmutativos por sus análogos no conmutativos y productos normales por productos estrella en la forma (10.8) de la acción de Born-Infeld no abeliana, donde la operación $\mathcal{S}$ se definirá según (10.9).

Por lo tanto, la acción de Born-Infeld no conmutativa quedará definida de acuerdo con $[127]$

$$
\begin{aligned}
& S_{B I}^{\theta}=-\beta^{2} \operatorname{Tr} \mathcal{S}^{*} \int d^{4} x\left(* \sqrt{1+\frac{1}{2 \beta^{2}} \hat{F}_{\mu \nu} * \hat{F}^{\mu \nu}-\frac{1}{16 \beta^{4}}\left(\hat{F}^{\mu \nu} * \tilde{\hat{F}}_{\mu \nu}\right)^{2}}-1\right)= \\
& =\operatorname{Tr} \mathcal{S}^{*} \int d^{4} x\left(-\frac{1}{4} \hat{F}_{\mu \nu} * \hat{F}^{\mu \nu}+\frac{1}{32 \beta^{2}}\left(\hat{F}^{\mu \nu} * \hat{\hat{F}}_{\mu \nu}\right)^{2}+\frac{1}{8}\left(\frac{1}{2 \beta} \hat{F}_{\mu \nu} * \hat{F}^{\mu \nu}-\frac{1}{16 \beta^{3}}\left(\hat{F}^{\mu \nu} * \hat{\hat{F}}_{\mu \nu}\right)^{2}\right)^{2}+\cdots\right),
\end{aligned}
$$

donde $* \sqrt{ }$ significa que la raíz cuadrada es definida usando el producto de Moyal en cada orden de la expansión en serie de potencias, y la operación $\mathcal{S}^{*}$ se ha definido de acuerdo con

$$
\mathcal{S}^{*}\left(\hat{F}_{\mu_{1} \nu_{1}} * \hat{F}_{\mu_{2} \nu_{2}} * \ldots * \hat{F}_{\mu_{N} \nu_{N}}\right)=\frac{1}{N !} \sum_{\{\sigma\}} \hat{F}_{\mu_{\sigma_{1}} \nu_{\sigma_{1}}} * \hat{F}_{\mu_{\sigma_{2}} \nu_{\sigma_{2}}} * \ldots * \hat{F}_{\mu_{\sigma_{N}} \nu_{\sigma_{N}}}
$$

Nótese que, si bien la operación compuesta $\operatorname{tr} \mathcal{S}^{*}$ coincide con la traza simétrica Str en el límite conmutativo $\theta_{\mu \nu} \rightarrow 0$, cuando $\theta_{\mu \nu}$ es no nulo la ecuación (10.10) deja de ser válida debido a la no conmutatividad del producto estrella entre las componentes de la curvatura en el álgebra de Lie $\hat{F}_{\mu \nu}^{a}$.

El orden simétrico definido en (10.15) asegura que los productos de campos reales como el lado derecho de (10.14), son números reales, lo cual no está garantizado para un producto estrella general no ordenado. $\mathcal{S}^{*}$ también resuelve las ambigüedades que surgen en la definición del lagrangiano DBI como un determinante y se puede probar que

$$
\begin{aligned}
S_{B I}^{\theta} & =\operatorname{Tr} \mathcal{S}^{*} \int d^{4} x\left(1-* \sqrt{1+\frac{1}{2}\left(\hat{F}^{\mu \nu} * \hat{F}_{\mu \nu}\right)-\frac{1}{16}\left(\hat{F}^{\mu \nu} * \tilde{\hat{F}}_{\mu \nu}\right)^{2}}\right)= \\
& =\operatorname{Tr} \mathcal{S}^{*} \int d^{4} x\left(1-* \sqrt{\operatorname{det}\left(g_{\mu \nu}+\hat{F}_{\mu \nu}\right)}\right)
\end{aligned}
$$

donde la última línea está unívocamente definida a través de la prescripción $\mathcal{S}^{*}$. Entonces, si se obtiene, partir de la construcción supersimétrica, una acción bosónica que toma la forma

ordinaria a través de un cambio de variables, impone ciertas condiciones en las correcciones en derivadas a la acción DBI, como se ha analizado en la referencia [102]-[105]. Otras aspectos de la acción DBI no conmutativa se han discutido en la literatura [106]-[110]. 
del lado izquierdo de (10.16), se puede escribir también sin ambigüedades como la forma usual de determinante.

Es fácil ver que (10.15) cambia covariantemente bajo transformaciones de gauge $\hat{g}$ cuando $\hat{F}_{\mu \nu} \rightarrow \hat{g}^{-1} * \hat{F}_{\mu \nu} * \hat{g}$

$$
\begin{aligned}
\operatorname{Tr} \mathcal{S}^{*}\left(\left[\hat{g}^{-1} * \hat{F}_{\mu_{1} \nu_{1}} * \hat{g}\right] *\left[\hat{g}^{-1} * \hat{F}_{\mu_{2} \nu_{2}} * \hat{g}\right] * \ldots *\left[\hat{g}^{-1} * \hat{F}_{\mu_{N} \nu_{N}} * \hat{g}\right]\right)= \\
\quad=\operatorname{Tr}\left(\frac{1}{N !} \sum_{\{\sigma\}} \hat{g}^{-1} * \hat{F}_{\mu_{\sigma_{1} \nu_{\sigma_{1}}}} * \hat{g} * \hat{g}^{-1} * \hat{F}_{\mu_{\sigma_{2} \nu_{\sigma_{2}}}} * \hat{g} * \ldots * \hat{g}^{-1} * \hat{F}_{\mu_{\sigma_{N}} \mu_{\sigma_{N}}} * \hat{g}\right)= \\
\quad=\operatorname{Tr}\left(\frac{1}{N !} \sum_{\sigma} \hat{g}^{-1} * \hat{F}_{\mu_{\sigma_{1} \nu_{\sigma_{1}}}} * \hat{F}_{\mu_{\sigma_{2} \nu_{\sigma_{2}}}} * \ldots * \hat{F}_{\left.\mu_{\sigma_{N} \nu_{\sigma_{N}}} * \hat{g}\right)=}\right. \\
\quad=\operatorname{Tr}\left(\hat{g}^{-1} * \mathcal{S}^{*}\left(\hat{F}_{\mu_{1} \nu_{1}}, \hat{F}_{\mu_{2} \nu_{2}}, \ldots, \hat{F}_{\mu_{N} \nu_{N}}\right) * \hat{g}\right) .
\end{aligned}
$$

Por lo tanto el lagrangiano definido con la prescripción $\mathcal{S}^{*}$ será covariante bajo transformaciones de gauge no conmutativas, lo que nos asegura que, con las condiciones de contorno adecuadas, la acción de Born-Infeld no conmutativa definida en (10.14) será invariante de gauge.

Nótese que en lugar de tomar la traza normal Tr en los generadores del álgebra de Lie, se podría intentar usar la traza simétrica STr, simetrizando el producto de generadores $t^{a}$, sin afectar los coeficientes $\hat{F}_{\mu \nu}^{a}$ (que podemos elegir simetrizar o no). Entonces, se puede ver que se obtiene una expresión diferente (la cual también se reduce a la prescripción de traza simétrica en el límite conmutativo). Sin embargo, esta receta para ordenar el producto se debe descartar ya que carece de la propiedad de covarianza de gauge. 


\title{
Capítulo 11
}

\section{Teoría de Born-Infeld supersimétrica en espacio no conmutativo}

\begin{abstract}
Presentamos una versión supersimétrica de la teoría de Born-Infeld en espacio-tiempo no conmutativo, para grupo de gauge abeliano y no abeliano. Mostramos, usando el formalismo de supercampos, que la definición del orden simétrico con respecto al producto estrella del que hablamos en el capítulo anterior lleva naturalmente a una acción de Born-Infeld supersimetrizable en ambos casos, el $U(1)$ y el $U(N)$. Analizamos las ecuaciones de Bogomol'nyi en este contexto y discutimos las propiedades de la teoría resultante.
\end{abstract}

\subsection{Supercampos en espacio no conmutativo}

Los supercampos se definen como las serie de potencias formales generadas por los objetos $(x, \theta, \bar{\theta})$ donde las $x^{\mu}$ son variables conmutantes, que se transforman en la representación vectorial frente al grupo de Lorentz y $\theta^{\alpha}, \bar{\theta}_{\dot{\alpha}}$ son variables anticonmutantes o de Grassman, que se transforman en la representación espinorial. Estos supercampos forman naturalmente un álgebra asociativa, que se puede identificar con el álgebra de funciones sobre el superespacio de coordenadas $(x, \theta, \bar{\theta})$.

Como se discute en [111], cuando se construyen deformaciones del álgebra de supercampos, existen dos parámetros libres que caracterizan la deformación, un parámetro $\theta^{\mu \nu}$ relacionado con el conmutador de las coordenadas vectoriales $x^{\mu}$ en la forma (2.4), y un nuevo parámetro asociado al anticonmutador de las coordenadas de Grassman $\theta^{\alpha}, \bar{\theta}_{\dot{\alpha}}$.

Nuestro interés en los supercampos se relaciona con la construcción de acciones supersimétricas para teorías de campos no conmutativas, para lo cual es suficiente considerar las deformaciones que afectan solamente al conmutador de las coordenadas $x^{\mu}$ de acuerdo a (2.4), sin modificar la regla de anticonmutación de las coordenadas de Grassman $\theta^{\alpha}, \bar{\theta}_{\dot{\alpha}}$ $[112],[113]$. 
Por lo tanto, definiremos el producto estrella entre supercampos $U_{1}, U_{2}$ de acuerdo a

$$
\left(U_{1} * U_{2}\right)(x, \bar{\theta}, \theta)=\left.e^{\frac{i}{2} \theta_{\mu \nu} \partial_{\xi^{\mu}} \partial_{\zeta^{\nu}}} U_{1}(x+\xi, \bar{\theta}, \theta) U_{2}(x+\zeta, \bar{\theta}, \theta)\right|_{\zeta=\xi=0},
$$

Nótese que las derivadas en el producto estrella involucran solo coordenadas de espacio tiempo, sin afectar a las coordenadas de Grassman del superespacio, que siguen siendo anticonmutantes cuando el conmutador se calcula con este nuevo producto.

Por lo tanto, la expansión de los supercampos en serie de potencias de $\theta^{\alpha}, \bar{\theta}_{\dot{\alpha}}$ es idéntica al caso conmutativo. Por ejemplo, un campo vectorial real $V=V^{\dagger}$ en el gauge de Wess-Zumino se escribe como

$$
V(x, \bar{\theta}, \theta)=-\theta^{\alpha} \sigma_{\alpha \dot{\beta}}^{\mu} \bar{\theta}^{\dot{\beta}} A_{\mu}+i \theta^{\alpha} \theta_{\alpha} \bar{\theta}_{\dot{\beta}} \bar{\lambda}^{\dot{\beta}}-i \bar{\theta}_{\dot{\beta}} \bar{\theta}^{\dot{\beta}} \theta^{\alpha} \lambda_{\alpha}+\frac{1}{2} \theta^{\alpha} \theta_{\alpha} \bar{\theta}_{\dot{\beta}} \bar{\theta}^{\dot{\beta}} D
$$

donde $D$ es el campo auxiliar

Es conveniente definir las variables quirales $y^{\mu}$ y $y^{\dagger^{\mu}}$ en la forma

$$
y^{\mu}=x^{\mu}+i \theta^{\alpha} \sigma_{\alpha \dot{\beta}}^{\mu} \bar{\theta}^{\dot{\beta}}, \quad y^{\dagger^{\mu}}=x^{\mu}-i \theta^{\alpha} \sigma_{\alpha \dot{\beta}}^{\mu} \bar{\theta}^{\dot{\beta}},
$$

de manera que se puedan definir las derivadas $D_{\alpha}$ and $\bar{D}_{\alpha}$ como

$$
D_{\alpha}=\frac{\partial}{\partial \theta^{\alpha}}+2 i\left(\sigma^{\mu} \bar{\theta}\right)_{\alpha} \frac{\partial}{\partial y^{\mu}}, \quad \bar{D}_{\dot{\alpha}}=-\frac{\partial}{\partial \bar{\theta}^{\dot{\alpha}}}-2 i\left(\theta \sigma^{\mu}\right)_{\dot{\alpha}} \frac{\partial}{\partial y^{\dagger \mu}} .
$$

Con la ayuda de estas variables quirales, un campo quiral izquierdo $\bar{D}_{\dot{\alpha}} \Lambda=0$ se escribe

$$
\Lambda(y, \theta)=A+\sqrt{2} \theta^{\alpha} \chi_{\alpha}+\theta^{\alpha} \theta_{\alpha} G,
$$

donde $A$ y $G$ son campos escalares complejos y $\chi$ es un espinor de Weil

Escribiremos las transformaciones de gauge generalizadas actuando sobre supercampos en la forma

$$
e_{*}^{2 i \Lambda}=1+2 i \Lambda-2 \Lambda * \Lambda+\cdots,
$$

donde $\Lambda=\Lambda(y, \theta)$ es un supercampo quiral izquierdo y $\Lambda^{\dagger}\left(y^{\dagger}, \bar{\theta}\right)$ su hermítico conjugado derecho $D_{\alpha} \Lambda^{\dagger}=0$. Bajo tal transformación el supercampo $V$ se transforma según

$$
e_{*}^{2 V} \rightarrow e_{*}^{-2 i \Lambda^{\dagger}} * e_{*}^{2 V} * e_{*}^{2 i \Lambda}
$$

A partir de $V$ se construye el supercampo quiral de curvatura,

$$
W^{\alpha}(y, \theta)=-\frac{1}{8} \bar{D}_{\dot{\alpha}} \bar{D}^{\dot{\alpha}} e_{*}^{-2 V} * D^{\alpha} e_{*}^{2 V} .
$$

En contraste con (11.7), bajo una transformación de gauge $W_{\alpha}$ se transforma covariantemente,

$$
W^{\alpha} \rightarrow e_{*}^{-2 i \Lambda} * W^{\alpha} * e_{*}^{2 i \Lambda} .
$$


En cuanto al conjugado hermítico, $\bar{W}_{\alpha}$, se transforma como

$$
\bar{W}_{\dot{\alpha}} \rightarrow e_{*}^{-2 i \Lambda^{\dagger}} * \bar{W}_{\dot{\alpha}} * e_{*}^{2 i \Lambda^{\dagger}}
$$

Escrito en componentes, $W_{\alpha}$ se lee

$$
W^{\alpha}(y, \theta)=-i \lambda^{\alpha}+\theta^{\alpha} D-\frac{i}{2}\left(\sigma^{\mu} \bar{\sigma}^{\nu} \theta\right)^{\alpha} F_{\mu \nu}+\theta \theta(\not \supset \bar{\lambda})^{\alpha} .
$$

Ahora estamos en condiciones de escribir acciones supersimétricas para el campo de gauge en espacio no conmutativo. Por ejemplo la acción supersimétrica $N=1$ de Yang-Mills no conmutativa se puede escribir en términos del supercampo quiral $W_{\alpha}$ y su conjugado hermítico $\bar{W}_{\dot{\alpha}}$ de acuerdo con

$$
S_{S Y M}^{\theta}=\frac{1}{4 e^{2}} \operatorname{Tr} \int d^{4} x\left(\int d^{2} \theta W^{\alpha} * W_{\alpha}+\int d^{2} \bar{\theta} \bar{W}_{\dot{\alpha}} * \bar{W}^{\dot{\alpha}}\right)
$$

ya que las últimas componentes en $W * W$ y $\bar{W} * \bar{W}$ contienen la combinación $D * D-$ $\frac{1}{2}\left(F_{\mu \nu} * F^{\mu \nu} \pm i F_{\mu \nu} * \tilde{F}^{\mu \nu}\right)$.

\subsection{La acción de Born-Infeld supersimétrica no con- mutativa}

Siguiendo un enfoque similar a [53], presentaremos aquí la versión supersimétrica de la teoría de Born-Infeld en espacio no conmutativo, para los casos con grupo de gauge abeliano y no abeliano. Usando el formalismo de supercampos, adaptado al caso no conmutativo de la manera que hemos descripto en la sección anterior, veremos que la definición del orden $\mathcal{S}^{*}$ con respecto a la multiplicación de Moyal lleva naturalmente a una acción de Born-Infeld tanto en el caso $U(1)$ como en el caso $U(N)$.

Con el objeto de construir una acción de Born-Infeld en espacio no conmutativo, se deben incluir invariantes que no pueden ser expresados en términos de los invariantes cuadráticos básicos $F_{\mu \nu} * F^{\mu \nu}$ y $F_{\mu \nu} * \tilde{F}^{\mu \nu}$. En efecto, si se busca un lagrangiano de Born-Infeld expresable como un determinante, aparecerán invariantes de Lorentz que no pueden ser escritos en términos de los cuadráticos que citamos arriba. Este es de hecho el caso de los términos cuárticos que necesariamente aparecerán cuando se computa el determinante de Born-Infeld en cuatro dimensiones: si bien en el caso conmutativo abeliano estos términos se pueden escribir como algún funcional de $F_{\mu \nu} F^{\mu \nu}$ y $F_{\mu \nu} \tilde{F}^{\mu \nu}$, en el caso no conmutativo el producto de Moyal evita tal acomodación. Mas aún, potencias impares, que están ausentes en el caso usual conmutativo, pueden ahora aparecer.

Empezaremos entonces la búsqueda de un candidato para la extensión supersimétrica del modelo de Born-Infeld en espacio no conmutativo. Sabemos que potencias mas altas de $W$ y 
$\bar{W}$ deben incluirse de manera tal de respetar la invarianza de Lorentz. En el caso del espacio conmutativo, esto se logra combinando $W^{2} \bar{W}^{2}$ con potencias adecuadas de $D^{2} W^{2}$ y $\bar{D}^{2} \bar{W}^{2}$ [99]-[100]. En el presente caso, hay dos fuentes de complicaciones. Primero en vista de las leyes de transformación (11.7) y (11.9)-(11.10), factores $\exp _{*}(2 \mathrm{~V})$ se deben incluir adecuadamente en términos cuárticos en los supercampos, de manera de asegurar la invarianza de gauge. Segundo, uno tiene que incluir tantos invariantes independientes de los supercampos como sea necesario para reproducir aquéllos invariantes de Lorentz cuárticos que no puedan escribirse en términos de $F_{\mu \nu} * F^{\mu \nu}$ y $F_{\mu \nu} * \tilde{F}^{\mu \nu}$.

Considérese entonces los supercampos invariantes de gauge que pueden originar términos cuárticos. Hay dos candidatos naturales

$$
\begin{aligned}
Q_{1} & =\int d^{2} \theta d^{2} \bar{\theta} W^{\alpha} * W_{\alpha} * e_{*}^{-2 V} * \bar{W}_{\dot{\beta}} * \bar{W}^{\dot{\beta}} * e_{*}^{2 V}, \\
Q_{2} & =\int d^{2} \theta d^{2} \bar{\theta} W^{\alpha} * e_{*}^{-2 V} * \bar{W}^{\dot{\beta}} * e_{*}^{2 V} * W_{\alpha} * e_{*}^{-2 V} * \bar{W}_{\dot{\beta}} * e_{*}^{2 V} .
\end{aligned}
$$

La parte bosónica de $Q_{1}$ está dada por

$$
\begin{aligned}
\left.Q_{1}\right|_{\text {bos }} & =D^{* 4}-\frac{1}{2}\left\{F^{\mu \nu} * F_{\mu \nu}, D^{* 2}\right\}-\frac{i}{2}\left[F^{\mu \nu} * \tilde{F}_{\mu \nu}, D^{* 2}\right] \\
& +\frac{1}{4}\left(\left(F^{\mu \nu} * F_{\mu \nu}\right)^{* 2}+\left(F^{\mu \nu} * \tilde{F}_{\mu \nu}\right)^{* 2}\right)+\frac{i}{4}\left[F^{\mu \nu} * \tilde{F}_{\mu \nu}, F^{\rho \sigma} * F_{\rho \sigma}\right],
\end{aligned}
$$

donde $A^{* 2}=A * A$. Si extraemos la parte dependiente sólo en $F_{\mu \nu}$ toma la forma

$$
\left.Q_{1}\right|_{b o s, F_{\mu \nu}}=\frac{1}{4}\left(\left(F^{\mu \nu} * F_{\mu \nu}\right)^{* 2}+\left(F^{\mu \nu} * \tilde{F}_{\mu \nu}\right)^{* 2}\right)+\frac{i}{4}\left[F^{\mu \nu} * \tilde{F}_{\mu \nu}, F^{\rho \sigma} * F_{\rho \sigma}\right] .
$$

Análogamente se obtiene para $Q_{2}$,

$$
\left.Q_{2}\right|_{b o s, F_{\mu \nu}}=\frac{1}{4} F^{\mu \nu} * F^{\rho \sigma} * F_{\mu \nu} * F_{\rho \sigma}+\frac{1}{4} \tilde{F}^{\mu \nu} * \tilde{F}^{\rho \sigma} * F_{\mu \nu} * F_{\rho \sigma}+\frac{i}{4}\left[F^{\mu \nu}, F^{\rho \sigma} * \tilde{F}_{\mu \nu} * F_{\rho \sigma}\right]
$$

Se puede ver ahora que una combinación muy económica de $Q_{1}$ y $Q_{2}$ genera los términos cuárticos que se esperan en la expansión de una acción DBI con raíz cuadrada, siempre que esta sea definida usando un orden simétrico de los factores $F_{\mu \nu}$. Efectivamente, bajo las condiciones de contorno adecuadas, que permitan rotar productos estrella bajo el signo integral, tenemos que

$$
\left.\frac{1}{4 !} \operatorname{Tr} \int d^{4} x\left(2 Q_{1}+Q_{2}\right)\right|_{b o s, F_{\mu \nu}}=\left.\operatorname{Tr} \mathcal{S}^{*} \int d^{4} x\left(1-* \sqrt{1+\frac{1}{2} F_{\mu \nu} * F^{\mu \nu}-\frac{1}{16}\left(F_{\mu \nu} * \tilde{F}^{\mu \nu}\right)^{* 2}}\right)\right|^{4^{\text {to }} \text { orden }},
$$


donde en el lado derecho hemos utilizado la operación de orden simétrico $\mathcal{S}^{*}$, definida como en el capítulo anterior

El análisis de arriba concierne a los términos puramente bosónicos. Es entonces natural extenderlo considerando la combinación de supercampos $\int d^{4} x\left(2 Q_{1}+Q_{2}\right)$, que nuevamente se puede acomodar en la forma de un orden simétrico, el cual ahora se debe definir de manera de tener en cuenta la presencia de objetos de Grassman anticonmutantes.

$$
\frac{1}{3} \operatorname{Tr} \int d^{4} x\left(2 Q_{1}+Q_{2}\right)=\operatorname{Tr} \mathcal{S}^{*} \int d^{4} x\left(W^{\alpha} * W_{\alpha} *\left[e_{*}^{-2 V} * \bar{W}_{\dot{\beta}} * e_{*}^{2 V}\right] *\left[e_{*}^{-2 V} * \bar{W}^{\dot{\beta}} * e_{*}^{2 V}\right]\right)
$$

donde ahora hemos definido el orden simétrico de supercampos de manera similar al caso puramente bosónico

$$
\mathcal{S}^{*}\left(U_{1} * U_{2} * \ldots * U_{n}\right)=\frac{1}{n !} \sum_{\{\pi\}} \operatorname{sgn} \pi_{O} U_{\pi(1)} * U_{\pi(2)} * \ldots * U_{\pi(n)} .
$$

Aquí $U_{i}$ representa a $W$ o $\left[\exp _{*}(-2 V) \bar{W} \exp _{*}(2 V)\right]$ (cualquiera de ambos) y sgn $\pi_{O}$ sólo toma en cuenta el signo de la permutación de objetos Grassman impares.

Nótese que en la ecuación (11.18), $\exp _{*}(2 V)$ y $\exp _{*}(-2 V)$ adyacentes dentro del símbolo $\mathcal{S}^{*}$, no se pueden cancelar antes de desarrollar el orden simétrico, ya que este desarrollo incluye términos en los cuales estos factores no son adyacentes. Es decir, las expresiones dentro de los paréntesis en (11.18) se deben tomar como elementos individuales al hacer las permutaciones.

Ahora, con el objeto de obtener las potencias más altas de $F_{\mu \nu} * F^{\mu \nu}$ y $F_{\mu \nu} * \tilde{F}^{\mu \nu}$ necesarias para construir la acción de Born-Infeld, se ha demostrado [99] que en el caso conmutativo se deben incluir potencias de ciertos supercampos, que llamaremos $X$ e $Y$, y que en el presente caso no conmutativo se deben definir como

$$
\begin{aligned}
X= & \frac{1}{16}\left(\left\{\bar{D}_{\dot{\beta}} \bar{D}^{\dot{\beta}} \bar{R}_{\dot{\alpha}}, \bar{R}^{\dot{\alpha}}\right\}-2 \bar{D}_{\dot{\beta}} \bar{R}_{\dot{\alpha}} * \bar{D}^{\dot{\beta}} \bar{R}^{\dot{\alpha}}+\left\{e_{*}^{-2 V} * D^{\beta} D_{\beta} R^{\alpha} * e_{*}^{2 V}, W_{\alpha}\right\}\right. \\
& \left.-2\left[e_{*}^{-2 V} * D^{\beta} R^{\alpha} * e_{*}^{2 V}\right] *\left[e_{*}^{-2 V} * D_{\beta} R_{\alpha} * e_{*}^{2 V}\right]\right), \\
Y= & -\frac{i}{32}\left(\left\{\bar{D}_{\dot{\beta}} \bar{D}^{\dot{\beta}} \bar{R}_{\dot{\alpha}}, \bar{R}^{\dot{\alpha}}\right\}-2 \bar{D}_{\dot{\beta}} \bar{R}_{\dot{\alpha}} * \bar{D}^{\dot{\beta}} \bar{R}^{\dot{\alpha}}-\left\{e_{*}^{-2 V} * D^{\beta} D_{\beta} R^{\alpha} * e_{*}^{2 V}, W_{\alpha}\right\}\right. \\
& \left.+2\left[e_{*}^{-2 V} * D^{\beta} R^{\alpha} * e_{*}^{2 V}\right] *\left[e_{*}^{-2 V} * D_{\beta} R_{\alpha} * e_{*}^{2 V}\right]\right),
\end{aligned}
$$

donde hemos definido

$$
\bar{R}_{\dot{\alpha}}=e_{*}^{-2 V} * \bar{W}_{\dot{\alpha}} * e_{*}^{2 V}, \quad R^{\alpha}=e_{*}^{2 V} * W^{\alpha} * e_{*}^{-2 V} .
$$

En particular, se tiene en el sector bosónico

$$
\left.X\right|_{\theta=\bar{\theta}=0}=-\frac{1}{2} D * D+\frac{1}{4} F^{\mu \nu} * F_{\mu \nu},
$$




$$
\left.Y\right|_{\theta=\bar{\theta}=0}=\frac{1}{8} F^{\mu \nu} * \tilde{F}_{\mu \nu}
$$

O sea que las componentes mas bajas de $X$ e $Y$ incluyen los invariantes $F_{\mu \nu} * F^{\mu \nu}$ y $F_{\mu \nu} * \tilde{F}^{\mu \nu}$.

Por lo tanto, esto no lleva a considerar la siguiente acción supersimétrica como un candidato para reproducir la dinámica de Born-Infeld en su sector bosónico

$$
S_{S B I}^{\theta}=S_{S Y M}^{\theta}+\sum_{n, m} C_{n m} \operatorname{Tr} \mathcal{S}^{*} \int d^{4} x d^{4} \theta\left(W^{\alpha} * W_{\alpha} * \bar{R}_{\dot{\beta}} * \bar{R}^{\dot{\beta}} * X^{* n} * Y^{* m}\right)+\text { h.c. }(
$$

La operación $\mathcal{S}^{*}$ fue definida en la ecuación (11.19) para supercampos $U$, esto implica, para los factores $X$ e $Y$, que se deben ordenar los paréntesis que entran en su construcción sin cambio de signo, ya que son Grassman pares.

La expresión (11.24) es de hecho bastante general. Es la extensión supersimétrica de una acción bosónica invariante de gauge que depende de la intensidad de campo $F_{\mu \nu}$ a través de las cantidades $F_{\mu \nu} * F^{\mu \nu}$ y $F_{\mu \nu} * \tilde{F}^{\mu \nu}$, en ciertas combinaciones restringidas por supersimetría. Los coeficientes $C_{m n}$ son arbitrarios y se pueden elegir de manera de obtener la acción de Born-Infeld en su forma usual. De hecho, si se pone

$$
C_{00}=\frac{1}{8}, \quad C_{n-2 m 2 m}=(-1)^{m} \sum_{j=0}^{m}\left(\begin{array}{c}
n+2-j \\
j
\end{array}\right) q_{n+1-j}, \quad C_{n 2 m+1}=0,
$$

donde

$$
q_{0}=-\frac{1}{2}, \quad q_{n}=\frac{(-1)^{n+1}}{4 n} \frac{(2 n-1) !}{(n+1) !(n-1) !} \quad \text { for } n \geq 1
$$

entonces, la parte puramente bosónica de la acción (11.24) se transforma, después de usar la relación (10.16),

$$
\left.S_{S B I}^{\theta}\right|_{b o s}=\operatorname{Tr} \mathcal{S}^{*} \int d^{4} x\left(1-* \sqrt{-\operatorname{det}\left(g_{\mu \nu}+F_{\mu \nu}\right)}\right)=S_{B I}^{\theta} .
$$

Como en el caso de espacio ordinario, existen otras elecciones posibles de los coeficientes $C_{m n}$ que llevan a una teoría de gauge supersimétrica, consistente con dinámica no polinómica para el campo de gauge. Déjesenos notar que las potencias impares de la intensidad de campo $F_{\mu \nu}$ han sido excluidas de nuestro tratamiento, ya que no es posible construir un funcional de los supercampos $W, \bar{W}, D W$ y $\bar{D} \bar{W}$ que contenga $F^{3}$ en su componente mas alta en $\theta$.

\subsection{Supersimetría $N=2$ y ecuaciones de Bogomol'nyi}

Un aspecto importante en el estudio de las acciones supersimétricas, concierne a las ecuaciones de Bogomol'niy-Prassad-Sommerfeld del sector bosónico. A este respecto, para 
teorías de born-Infeld conmutativas, han sido estudiadas las condiciones bajo las cuales las ecuaciones resultantes coinciden con las de la teoría de Yang-Mills, y ha sido aclarada su relación con la supersimetría $N=2$ [45]-[47], [101]. En el caso no conmutativo, se han encontrado ya soluciones a diferentes ecuaciones autoduales [116]-[124], pero falta el análisis de la conexión entre estas ecuaciones y la supersimetría. Es entonces natural investigar esta cuestión en el marco supersimétrico aquí presentado, extendiendo nuestro tratamiento de supersimetría $N=1$ a $N=2$.

Para llevar a cabo la extensión al caso $N=2$, se debe añadir al supermultiplete vectorial $V$ ya introducido, un supermuliplete escalar quiral $\Phi(y, \theta)=\phi+\sqrt{2} \theta^{\alpha} \psi_{\alpha}+\theta^{\alpha} \theta_{\alpha} F$, donde $\phi=\phi_{1}+i \phi_{2}$ es un campo escalar complejo, $\psi$ un segundo fermión de Majorana, y $F$ un campo auxiliar complejo. Un multiplete vectorial completo $N=2$ se puede acomodar en términos de todos estos campos en la forma $\left(A_{\mu}, \chi, \phi, D, F\right)$, donde ahora $\chi$ es un fermión de Dirac construido a partir de $\lambda$ y $\psi, \chi=(\lambda, \bar{\psi})$.

El lagrangiano de Born-Infeld $N=2$ se construye siguiendo los mismos pasos descriptos para el caso $N=1$. Se usa como bloque para la construcción el supercampo quiral $N=2$, $\Psi$, el cual juega un rol análogo al del supercampo de curvatura $W$ en el caso $N=1$.

$$
\Psi=\Phi\left(\tilde{y}, \theta_{1}\right)+i \sqrt{2} \theta_{2}{ }^{\alpha} W_{\alpha}\left(\tilde{y}, \theta_{1}\right)+\theta_{2}^{\alpha} \theta_{2 \alpha} G\left(\tilde{y}, \theta_{1}\right),
$$

aquí $\tilde{y}^{\mu}=y^{\mu}+i \theta_{2} \sigma^{\mu} \bar{\theta}_{2}$ y $G$ es un escalar quiral, el cual se puede expresar en términos de $\Phi$ y $V$ (véase por ejemplo [115])

Es fácil ver que

$$
\left.\Psi^{2}\right|_{\psi=\lambda=\phi=0}=-4 \theta_{1}^{2} \theta_{2}^{2}\left(F_{\mu \nu} * F^{\mu \nu}+i F_{\mu \nu} * \tilde{F}^{\mu \nu}-\frac{1}{2} D * D-\frac{1}{4}\left\{F^{\dagger}, F\right\}_{+}\right)
$$

y por lo tanto la parte bosónica del lagrangiano $N=2$ dependerá de $F_{\mu \nu} D$ y $F$ a través de la combinación arriba definida. Entonces, las ecuaciones de movimiento para $D$ y $F$ son satisfechas para $D=F=0$.

Con el objeto de buscar configuraciones de instantón, se debe pasar a espacio euclídeo e igualar a cero la ley de transformación de supersimetría para el gaugino. Cuando suponemos $\theta^{0 i}=0$, la rotación de Wick se puede llevar a cabo sin ninguna complicación adicional relacionada con la definición del producto estrella. Ahora, en la ley de transformación del gaugino, igualaremos a cero todos los campos fermiónicos y el campo escalar se deben poner a cero mientras que los campos auxiliares $D$ y $F$ se deben eliminar usando sus ecuaciones de movimiento, las cuales, como hemos visto, llevan a $D=F=0$.

La ley de transformación de supersimetría del gaugino toma la forma

$$
\delta \chi=-i\left(\frac{i}{4}\left[\gamma^{\mu}, \gamma^{\nu}\right] F_{\mu \nu}-D\right) \xi+i \sqrt{2} \not \partial\left(\gamma_{5} \phi_{1}+i \phi_{2}\right) \chi-\sqrt{2} F^{\dagger} \gamma_{5} \bar{\chi}^{t}
$$

donde $\left(\xi_{1},-\bar{\xi}_{2}\right)=\xi$ son los dos parámetros de transformación de la supersimetría $N=2$. Poniendo $\phi=D=F=0$, la ley de transformación del gaugino se reduce a

$$
\delta \chi=-i \Gamma^{\mu \nu} F_{\mu \nu} \xi
$$


por lo tanto, cuando se imponen las condiciones de Bogomol'niy-Prassad-Sommerfeld en la forma $\delta \chi=0$, se obtiene la ecuación de autodualidad, la cual en espacio euclídeo toma la forma

$$
F_{\mu \nu}= \pm \tilde{F}_{\mu \nu}
$$

Cada una de las soluciones rompen la mitad de las supersimetrías. Como se esperaba, la ecuación del instantón coincide formalmente con la que surge en espacio tiempo ordinario. Por supuesto, se debe recordar que $F_{\mu \nu}$ en la ecuación (11.32) como se definió en (4.12) incluye un conmutador de Moyal. Esto significa que aún en el caso $U(1)$ podría haber soluciones de instantón, las cuales de hecho se han encontrado en [116].

\subsection{Alcances y limitaciones}

- Para entender mas profundamente las cotas y ecuaciones BPS, deberíamos construir el álgebra de supersimetría $N=2$.

- Sería interesante clarificar la relación de nuestra prescripción de ordenamiento simétrico con otras propuesta para la acción de Born-Infeld no conmutativa mencionadas en la literatura [102]-[105].

- Deberíamos estudiar el comportamiento de la acción de Born-Infeld no conmutativa que hemos definido bajo el mapeo de Seiberg y Witten, así como su relación con la teoría de cuerdas.

\subsection{Conclusiones}

Como sumario de nuestras conclusiones podemos decir lo siguiente

- El problema de definir un acción escalar a partir de objetos valuados en el álgebra de Lie, se reduce simplemente a tomar la traza de la expresión simetrizada con $\mathcal{S}^{*}$. Como hemos demostrado, esta prescripción lleva a una acción de Born-Infeld que es invariante de gauge, en contraste con lo que pasaría si usáramos la traza simétrica después de simetrizar sobre los coeficientes. Aún así, en el límite $\theta_{\mu \nu} \rightarrow 0$, debido al efecto de simetrización de $\mathcal{S}^{*}$, nuestro resultado coincide con el obtenido para la acción de Born-Infeld no abeliana conmutativa usando la traza simétrica

- Hemos construido la extensión supersimétrica de la acción de Born-Infeld no conmutativa. En nuestra construcción, hemos visto que las funcionales naturales de los supercampos a partir de las cuales se construyen las extensiones supersimétricas de teorías de gauge, se combinan en la forma adecuada de raíz cuadrada de la acción de Born-Infeld, de manera tal que el orden simétrico $\mathcal{S}^{*}$ es señalado. 
- Hemos avanzado en la construcción de una acción de Born-Infeld supersimétrca $N=2$ no conmutativa, verificando que las condiciones de Bogomol'niy-Prassad-Sommerfeld que de ella se obtienen, coinciden con las de la teoría de Yang-Mills no conmutativa. 


\section{Capítulo 12}

\section{Conclusiones}

En esta tesis doctoral hemos estudiado dos teorías de gauge definidas en espacio no conmutativo: la teoría de Chern-Simons y la de Born-Infeld. Tanto por su simplicidad cuanto por sus posibles aplicaciones en modelos físicos de interés, hemos seleccionado un tipo especial de espacio no conmutativo conocido como $\theta$-deformado.

En lo que concierne a la acción de Chern-Simons $\theta$-deformada, inducidos por los celebrados resultados obtenidos en espacio conmutativo [24], hemos investigado la posibilidad de que el término de Chern-Simons se genere por fluctuaciones de fermiones masivos en $2+1$ dimensiones espacio temporales, en las diferentes representaciones del grupo de gauge. $\mathrm{Pu}-$ dimos mostrar que cuando se toman en cuenta las contribuciones del regulador se obtiene un resultado invariante de gauge, aún bajo transformaciones "grandes". En todos los casos, la forma de la parte que viola paridad de la acción efectiva invariante de gauge, es la de una acción de Chern-Simons no conmutativa.

Para las representaciones fundamental y anti-fundamental, el cálculo es completamente análogo al caso conmutativo, ya que las únicas contribuciones a la acción efectiva provienen de diagramas planos, en los que el factor de fase sale fuera de las integrales. Para la representación adjunta, en la cual intervienen diagramas no planos, el resultado invariante de gauge no trivial (6.20) es originado solamente por los campos reguladores, lo que implica que el límite conmutativo $\theta_{\mu \nu} \rightarrow 0$ no conmuta con el límite de la regularización $M \rightarrow \infty$. Este fenómeno se conoce como mezcla infrarrojo-ultravioleta y es el análogo tridimensional del resultado obtenido por A. Matusis, L. Susskind y N. Toumbas en [79], para el caso de la anomalía quiral en $d=3+1$.

Luego, en vista de posibles aplicaciones al efecto Hall propuestas en [22], hemos considerado la posibilidad de definir la acción de Chern-Simons en un espacio con borde, determinando las condiciones de contorno adecuadas para que sea diferenciable e invariante de gauge. A este respecto encontramos dos casos relevantes: un caso especial en el cual existen bordes sobre los cuales $\theta^{\mu \nu}$ no tiene componentes normales, de modo que sobre ellos es necesario y 
suficiente para la diferenciabilidad de la acción que se anule una componente tangencial del campo de gauge; y un caso general, en el cual sobre aquellos bordes en los cuales $\theta^{\mu \nu}$ tenga componentes normales no nulas, la condición de contorno a imponer es que dos componentes del campo de gauge, junto con sus infinitas derivadas normales, sean cero.

A continuación hemos encontrado las transformaciones de simetría que preservan las condiciones de contorno, las cuales verifican las condiciones adecuadas en el borde, a saber: para el caso especial antes citado, las simetrías de la teoría serán las funciones valuadas en el grupo de gauge, tales que sobre el borde no dependan de una dirección tangente; para el caso general, las funciones no deben depender de dos direcciones en el borde, y todas sus derivadas normales deben anularse.

Hemos investigado también la relación entre la acción de Chern-Simons y la acción quiral de Wess-Zumino-Witten definida en el borde de la variedad, un aspecto importante, que en el caso conmutativo es muy útil para el análisis de modelos invariantes conformes de interés en mecánica estadística. Concluímos que cuando la variedad tiene la topología del disco por la recta real, la acción de Chern-Simons es equivalente a un modelo de Wess-Zumino-Witten con las siguientes características: para el caso especial, obtenemos una acción quiral de WessZumino-Witten, con los productos ordinarios transformados en productos estrella. Es decir obtenemos lo que llamaríamos un modelo quiral de Wess-Zumino-Witten no conmutativo; para el caso general, obtenemos un modelo de Wess-Zumino-Witten no conmutativo un poco más complicado, debido a la necesidad de imponer condiciones de contorno sobre dos componentes del campo de gauge en lugar de sobre una sola.

Motivados por la relación entre la acción de Chern-Simons y el modelo quiral de WessZumino-Witten, y la equivalencia entre los modelos de Wess-Zumino-Witten conmutativo y no conmutativo, investigamos el comportamiento de la acción de Chern-Simons bajo el mapeo de Seiberg y Witten, que relaciona teorías no conmutativas con diferentes valores del parámetro de no conmutatividad $\theta^{\mu \nu}$. Demostramos que, a diferencia de otros casos, el modelo de Chern-Simons se mapea en un modelo análogo, independientemente del valor de este parámetro.

A continuación hemos formulado una teoría de Chern-Simons no conmutativa, con grupo de gauge $G L(2, \mathbb{R})$, con la idea de dar una definición de la gravitación no conmutativa. Es sabido que en el caso conmutativo, la gravedad en $d=3$ dimensiones euclídeas es equivalente a una teoría de Chern-Simons con grupo de gauge $S L(2, \mathbb{C})$. El límite conmutativo de nuestra teoría coincide, a nivel clásico, con dicha forma de Chern-Simons de la gravitación, unida a un campo de gauge $U(1)$ complejo, desacoplado y con acción de Chern-Simons. Para $\theta^{\mu \nu}$ finito, el acoplamiento entre el campo $U(1)$ y la parte $S L(2, \mathbb{R})$ es no trivial.

Hemos sido capaces de reformular dicha teoría de Chern-Simons en términos de un dreibein y conexión de spin no conmutativos, cuyas ecuaciones de movimiento generalizan las ecuaciones de estructura de Cartan. Verificamos que es posible definir una acción de Einstein no conmutativa, escrita en términos de los campos no conmutativos, de la cual se derivan 
las correctas ecuaciones de movimiento, sin necesidad de definir algún ordenamiento para el determinante del dreibein, sorteando de esta manera las dificultades que se han discutido en la literatura [87]-[90]. Además, hemos formulado estas ecuaciones ne términos de métrica y conexión afín, encontrando los que hemos definido como las ecuaciones de Einstein no conmutativas.

Encontramos soluciones a nuestras ecuaciones que corresponden, en el límite conmutativo, a las soluciones conocidas de la teoría de gravitación en $d=3$ dimensiones, en particular la solución afín y la solución de agujero negro BTZ. Además, hemos encontrado un operador que mapea las soluciones de nuestra teoría no conmutativa en las correspondientes soluciones de la teoría conmutativa.

En cuanto a la teoría de Born-Infeld, dada su utilidad en el contexto de la teoría de cuerdas para la descripción de la dinámica de baja energía de las $D$-branas, nos propusimos definir la correspondiente versión no conmutativa, para los casos con grupo de gauge abeliano y no abeliano. En nuestra construcción, hemos encontrado que el orden simétrico de los factores $\hat{F}_{\mu \nu}$ en el desarrollo en serie de potencias de la acción, provee una definición adecuada para la acción de Born-Infeld invariante de gauge.

Esta construcción se puede hacer independientemente del grupo de gauge. Remarcablemente, en el caso $U(N)$, el problema de definir una acción escalar a partir de objetos valuados en el álgebra de Lie, se reduce simplemente a tomar la traza de la expresión simetrizada. Como demostramos, esta prescripción lleva a una acción de Born-Infeld que es invariante de gauge en contraste con lo que pasaría si usáramos la traza simétrica. Aún así, en el límite conmutativo, debido al efecto de la simetrización, nuestro resultado coincide con el obtenido para la acción de Born-Infeld no abeliana conmutativa, cuando se usa la traza simétrica [44].

La extensión supersimétrica de la acción de Born-Infeld que construímos, se basa en el formalismo de supercampos, adecuadamente extendido al presente caso no conmutativo. La acción resultante conduce a un sector bosónico con dinámica gobernada por la acción de Born-Infeld no conmutativa, a todos los órdenes en $\theta^{\mu \nu}$.

Finalmente, hemos hecho algunas consideraciones sobre la extensión supersimétrica $N=$ 2 y las ecuaciones de Bogomol'nyi de la acción de Born-Infeld no conmutativa. Como se esperaba, la ecuación de autodualidad coincide formalmente con la que surge en espacio tiempo ordinario. es de interés recordar que, dado que el tensor de curvatura no conmutativo incluye un conmutador de Moyal, pueden existir soluciones de instantón aún en el caso $U(1)$, las que de hecho han sido encontradas en [116].

En resumen, hemos estudiado la acción de Chern-Simons en espacio no conmutativo, demostrando que se puede obtener como la acción efectiva para fermiones masivos en $d=$ $2+1$ dimensiones, y hallando las condiciones en las cuales esta acción esta bien definida en variedades con borde, además de estudiar su relación con el modelo quiral de Wess-ZuminoWitten y con la acción de Chern-Simons usual conmutativa. También la hemos aplicado a 
la construcción de una teoría de gravitación tridimensional no conmutativa, encontrando soluciones de las ecuaciones clásicas de movimiento, entre las que se encuentra el agujero negro tridimensional BTZ.

Además, hemos definido una acción de Born-Infeld no conmutativa, en la cual un ordenamiento simétrico de los factores que contienen la curvatura de gauge, asegura la invarianza de la acción. Construímos además una extensión supersimétrica, verificando que la supersimetría es consistente con el ordenamiento simétrico y la hemos utilizado para investigar las ecuaciones de Bogomol'nyi del sector bosónico.

Como hemos hecho al terminar cada capítulo, señalaremos aquí los problemas abiertos por nuestro trabajo, que pueden ser objeto de una futura investigación 6.4.

- Generalizando nuestros cálculos sobre la acción de Chern-Simons inducida, se podría considerar la contribución de gráficos con dos o mas loops, y verificar si existe una versión no conmutativa del teorema de no renormalización de Coleman y Hill [30]. Tal demostración deberia seguir las líneas de las versiones no abelianas [31], [32], de manera similar a lo desarrollado en [77].

- Es natural preguntarse acerca de los diagramas de un loop, pero con más patas externas, que no contribuyen al término de Chern-Simons ya calculada. En el caso masivo, estos gráficos, si bien continúan siendo convergentes y por lo tanto no reciben contribuciones que contengan la masa del regulador, contienen potencias de la masa física $m$. Por lo tanto no está en principio vedado que contribuyan a la violación de paridad.

- El siguiente orden en la expansión en potencias de $p /|m|$, corresponde en el caso usual conmutativo abeliano a la extensión de Chern-Simons en derivadas mas altas, estudiada en [28],[29]. En el caso no conmutativo la fórmula análoga no es invariante de gauge. La forma de una extensión en derivadas más altas, invariante de gauge, contiene términos cuadráticos en $\hat{A}_{\mu}$ y en $\hat{F}_{\mu}$, por lo que provendrá necesariamente de gráficos con más patas externas. Sería interesante verificar si la forma invariante de gauge de la extensión de Chern-Simons en derivadas más altas, se obtiene al calcular explícitamente las contribuciones de los gráficos adecuados.

- En nuestro trabajo sobre la acción de Chern-Simons en variedades con borde utilizamos la expresión diferencial del producto de Moyal. Es importante verificar si los resultados relativos a a las condiciones de borde se sostienen cuando se utiliza la forma integral de este producto, que depende fuertemente de la variedad elegida.

- Sería interesante estudiar el efecto del mapeo de Seiberg y Witten sobre otras teorías de gauge no conmutativas cuya ecuación clásica de movimiento sea $\hat{F}_{\mu \nu}=0$, como por ejemplo los modelos $B F$. 
- Queda por realizar un análisis cuántico de este mapeo, lo que requeriría el del comportamiento de la medida de integración bajo este cambio de variables.

- Es importante estudiar la relación de nuestro modelo de gravitación no conmutativa con otras propuestas como las que se han discutido en [9]-[14] y en [87]-[90]

- Sería interesante encontrar una interpretación geométrica del término adicional en las ecuaciones de Einstein no conmutativas.

- En lo relacionado con la acción de Born-Infeld, es interesante clarificar la relación de nuestra prescripción de ordenamiento simétrico con otras propuestas para la acción de Born-Infeld no conmutativa mencionadas en la literatura [102]-[105].

- Queda abierta la posibilidad de estudiar el comportamiento de la acción de Born-Infeld no conmutativa que hemos definido bajo el mapeo de Seiberg y Witten, así como su relación con la teoría de cuerdas. 


\section{Parte IV \\ Apéndices}





\section{Capítulo 13}

\section{Algunas propiedades del producto estrella}

1. Conjugación compleja: El complejo conjugado del producto estrella de dos funciones $f$ y $g$ es igual al producto de las funciones conjugadas, tomado en orden inverso

$$
\begin{aligned}
(f * g)^{*} & =\left(\sum_{n=0}^{\infty} \frac{1}{n !}\left(\frac{i}{2}\right)^{n} \theta^{\alpha_{1} \beta_{1}} \ldots \theta^{\alpha_{n} \beta_{n}} \partial_{\alpha_{1}} \ldots \partial_{\alpha_{n}} f \partial_{\beta_{1}} \ldots \partial_{\beta_{n}} g\right)^{*}= \\
& =\sum_{n=0}^{\infty} \frac{1}{n !}\left(-\frac{i}{2}\right)^{n} \theta^{\alpha_{1} \beta_{1}} \ldots \theta^{\alpha_{n} \beta_{n}} \partial_{\alpha_{1}} \ldots \partial_{\alpha_{n}} f^{*} \partial_{\beta_{1}} \ldots \partial_{\beta_{n}} g^{*} \\
& =\sum_{n=0}^{\infty} \frac{1}{n !}\left(-\frac{i}{2}\right)^{n}(-1)^{n} \theta^{\beta_{1} \alpha_{1}} \ldots \theta^{\beta_{n} \alpha_{n}} \partial_{\beta_{1}} \ldots \partial_{\beta_{n}} g^{*} \partial_{\alpha_{1}} \ldots \partial_{\alpha_{n}} f^{*} \\
& =g^{*} * f^{*}
\end{aligned}
$$

2. Relación con el producto normal: el producto estrella de dos funciones $f$ y $g$ se puede escribir como el producto puntual usual de ambas funciones mas una derivada total

$$
\begin{aligned}
f * g= & \sum_{n=0}^{\infty} \frac{1}{n !}\left(\frac{i}{2}\right)^{n} \theta^{\alpha_{1} \beta_{1}} \ldots \theta^{\alpha_{n} \beta_{n}} \partial_{\alpha_{1}} \ldots \partial_{\alpha_{n}} f \partial_{\beta_{1}} \ldots \partial_{\beta_{n}} g= \\
= & f g+\sum_{n=1}^{\infty} \frac{1}{n !}\left(\frac{i}{2}\right)^{n} \theta^{\alpha_{1} \beta_{1}} \ldots \theta^{\alpha_{n} \beta_{n}} \partial_{\alpha_{1}} \ldots \partial_{\alpha_{n}} f \partial_{\beta_{1}} \ldots \partial_{\beta_{n}} g= \\
= & f g+ \\
& +\theta^{\alpha_{1} \beta_{1}} \partial_{\alpha_{1}} \sum_{n=1}^{\infty} \frac{1}{n !}\left(\frac{i}{2}\right)^{n} \theta^{\alpha_{2} \beta_{2}} \ldots \theta^{\alpha_{n} \beta_{n}} \partial_{\alpha_{2}} \ldots \partial_{\alpha_{n}} f \partial_{\beta_{1}} \ldots \partial_{\beta_{n}} g= \\
= & f g+\partial_{\mu} B^{\mu}[f, g]
\end{aligned}
$$


donde

$$
B^{\mu}[f, g]=\theta^{\mu \beta_{1}} \sum_{n=1}^{\infty} \frac{1}{n !}\left(\frac{i}{2}\right)^{n} \theta^{\alpha_{2} \beta_{2}} \ldots \theta^{\alpha_{n} \beta_{n}} \partial_{\alpha_{2}} \ldots \partial_{\alpha_{n}} f \partial_{\beta_{1}} \ldots \partial_{\beta_{n}} g
$$

3. Conmutador de Moyal: el conmutador de dos funciones $f$ y $g$ es una derivada total, como se deduce fácilmente de la formula anterior

$$
[f, g]-=\partial_{\mu} B^{\mu}[f, g]
$$

donde

$$
\begin{aligned}
B^{\mu}[f, g]= & B^{\mu}[f, g]-B^{\mu}[g, f]= \\
= & \theta^{\mu \beta_{1}} \sum_{n=1}^{\infty} \frac{1}{n !}\left(\frac{i}{2}\right)^{n} \theta^{\alpha_{2} \beta_{2}} \ldots \theta^{\alpha_{n} \beta_{n}} \partial_{\alpha_{2}} \ldots \partial_{\alpha_{n}} f \partial_{\beta_{1}} \ldots \partial_{\beta_{n}} g- \\
& -\theta^{\mu \beta_{1}} \sum_{n=1}^{\infty} \frac{1}{n !}\left(\frac{i}{2}\right)^{n} \theta^{\alpha_{2} \beta_{2}} \ldots \theta^{\alpha_{n} \beta_{n}} \partial_{\alpha_{2}} \ldots \partial_{\alpha_{n}} g \partial_{\beta_{1}} \ldots \partial_{\beta_{n}} f= \\
& =\theta^{\mu \beta_{1}} \sum_{n=1}^{\infty} \frac{1}{n !}\left(\frac{i}{2}\right)^{n} \theta^{\alpha_{2} \beta_{2}} \ldots \theta^{\alpha_{n} \beta_{n}} \partial_{\alpha_{2}} \ldots \partial_{\alpha_{n}} f \partial_{\beta_{1}} \ldots \partial_{\beta_{n}} g- \\
- & \theta^{\mu \beta_{1}} \sum_{n=1}^{\infty} \frac{1}{n !}(-1)^{n-1}\left(\frac{i}{2}\right)^{n} \theta^{\alpha_{2} \beta_{2}} \ldots \theta^{\alpha_{n} \beta_{n}} \partial_{\beta_{2}} \ldots \partial_{\beta_{n}} g \partial_{\beta_{1}} \partial_{\alpha_{2}} \ldots \partial_{\alpha_{n}} f= \\
& =\theta^{\mu \beta_{1}} \sum_{n=1}^{\infty} \frac{1}{n !}\left(\frac{i}{2}\right)^{n} \theta^{\alpha_{2} \beta_{2}} \ldots \theta^{\alpha_{n} \beta_{n}} \partial_{\alpha_{2}} \ldots \partial_{\alpha_{n}} f \partial_{\beta_{1}} \ldots \partial_{\beta_{n}} g- \\
- & \theta^{\mu \beta_{1}} \sum_{n=1}^{\infty} \frac{1}{n !}(-1)^{n}\left(\frac{i}{2}\right)^{n} \theta^{\alpha_{2} \beta_{2}} \ldots \theta^{\alpha_{n} \beta_{n}} \partial_{\beta_{1}} \ldots \partial_{\beta_{n}} g \partial_{\alpha_{2}} \ldots \partial_{\alpha_{n}} f= \\
& =\theta^{\mu \beta_{1}} \sum_{n=1}^{\infty} \frac{1}{n !}\left(1-(-1)^{n}\right)\left(\frac{i}{2}\right)^{n} \theta^{\alpha_{2} \beta_{2}} \ldots \theta^{\alpha_{n} \beta_{n}} \partial_{\alpha_{2}} \ldots \partial_{\alpha_{n}} f \partial_{\beta_{1}} \ldots \partial_{\beta_{n}} g= \\
& =\theta^{\mu \beta_{1}} \sum_{n \text { impar }}^{\infty} \frac{1}{n !}\left(\frac{i}{2}\right)^{n} \theta^{\alpha_{2} \beta_{2}} \ldots \theta^{\alpha_{n} \beta_{n}} \partial_{\alpha_{2}} \ldots \partial_{\alpha_{n}} f \partial_{\beta_{1}} \ldots \partial_{\beta_{n}} g
\end{aligned}
$$

4. Permutación cíclica: la permutación cíclica de un producto estrella genera derivadas totales

$$
f * g * h=h * f * g+\partial_{\mu} B_{\text {Cicl }}^{\mu}[f, g, h]
$$

donde

$$
B_{\mathrm{Cicl}}^{\mu}[f, g, h]=B^{\mu}[f * g, h]=B^{\mu}[f, g * h]+B^{\mu}[g, h * f]
$$




\section{Capítulo 14}

\section{Notación}

- Conjuntos

$\Omega$

$\mathcal{C}_{0}(\Omega)$

$\mathcal{C}$

$\mathcal{A}$

$\mathcal{M}$

$\mathcal{C}_{\theta}(\mathcal{M})$ espacio topologico,

funciones continuas definidas sobre $\Omega$,

algebra conmutativa arbitraria,

algebra no conmutativa arbitraria,

variedad arbitraria

algebra de funciones $\theta$-deformada sobre la variedad $\mathcal{M}$

- Subíndices:

$$
\begin{aligned}
& A, B \in[0, . ., 9] \\
& \mu, \nu, \rho, \sigma, \epsilon \in[0, . ., d<9] \\
& i, j, k \in[1, d] \\
& \alpha, \beta, \gamma \in[0,1] \\
& a, b, c
\end{aligned}
$$

dimensiones del espacio tiempo, dimensiones del espacio tiempo $d=2$ en la Parte II y $d=3$ en la Parte III dimensiones espaciales del espacio tiempo dimensiones de la hoja de mundo indices de gauge

- Operaciones

$$
\begin{aligned}
& \cdots * \cdots \\
& \{\cdots, \cdots\}_{P B} \\
& {[\cdots, \cdots]} \\
& \{\cdots, \cdots\}_{D B} \\
& \langle\cdots\rangle \\
& \left.\cdots\right|_{\text {impar }} \\
& \operatorname{Tr}(\cdots) \\
& \operatorname{tr}(\cdots)
\end{aligned}
$$

producto estrella, parentesis de Poisson, conmutador de Moyal, parentesis de Dirac, valor de expectacion de vacio toma la parte no invariante de paridad de la expresion, representa la traza sobre los indices del grupo de gauge representa traza sobre indices espinoriales 


\section{Bibliografía}

[1] P.A.M. Dirac, The fundamental equations of quantum mechanics, Proc. Roy. Soc. A109 (1926) 642;

[2] P.A.M. Dirac, On quantum algebras, Proc. Camb. Phil. Soc. 23 (1926) 412.

[3] H. S. Snyder, Quantized Space-Time, Phys. Rev. 71 (1947) 38.

[4] H. S. Snyder, The electromagnetic field in quantized space-time, Phys. Rev. 72 (1947) 68.

[5] A. Connes Géométrie non commutative (1990) InterEditions, Paris. Para una versión en inglés ver Noncommutative Geometry (1994) Academic Press

[6] A. Connes and J. Lott, Particle Models and Noncommutative Geometry, Nucl. Phys. (Proc. Suppl.) B18 (1990) 29;

[7] J.C. Várilly and J.M. Gracia-Bondía, Connes' Noncommutative Differential Geometry and the Standard Model, J. Geom. Phys. 12 (1993) 223;

[8] C.P. Martín, J.M. Gracia-Bondía and J.C. Várilly, The Standard Model as a Noncommutative Geometry: The Low-Energy Regime, Phys. Rep. 294 (1998) 363 [hep-th/9605001].

[9] A.H. Chamseddine, G. Felder and J. Fröhlich, Gravity in Noncommutative Geometry, Commun. Math. Phys. 155 (1993) 205 [hep-th/9209044];

[10] W. Kalau and M. Walze, Gravity, Noncommutative Geometry and the Wodzicki Residue, J. Geom. Phys. 16 (1995) 327 [gr-qc/9312031];

[11] D. Kastler, The Dirac Operator and Gravitation, Commun. Math. Phys. 166 (1995) 633 ;

[12] A.H. Chamseddine, J. Fröhlich and O. Grandjean, The Gravitational Sector in the Connes-Lott Formulation of the Standard Model, J. Math. Phys. 36 (1995) 6255 [hep-th/9503093]; 
[13] A. Connes, Gravity coupled with matter and the foundation of non-commutative geometry, Commun. Math. Phys. 182 (1996) 155 [hep-th/9603053].

[14] A.H. Chamseddine and A. Connes, The Spectral Action Principle, Commun. Math. Phys. 186 (1997) 731 [hep-th/9606001].

[15] B. DeWitt, Gravitation, Edited by L.Witten, (1962) 266-381

[16] E. Witten, Noncommutative Geometry And String Field Theory, Nucl. Phys. B 268 (1986) 253.

[17] A. Connes, M. R. Douglas y A. Schwarz, Noncommutative geometry and matrix theory: Compactification on tori, JHEP 9802 (1998) 003 [hep-th/9711162].

[18] M. R. Douglas y C. M. Hull, D-branes and the noncommutative torus, JHEP 9802 (1998) 008 [hep-th/9711165].

[19] N. Seiberg y E. Witten, String theory and noncommutative geometry, JHEP 9909 (1999) 032 [hep-th/9908142].

[20] S. A. Girvin and R. Prange The quantum Hall effect (1987)

[21] J. Bellissard, A. Van Elst and H. Schulz-Valdes The noncommutative geometry and the quantum Hall effect [cond-mat/9301005]

[22] L. Susskind, The quantum Hall fluid and non-commutative Chern Simons theory, [hep-th/0101029].

[23] S. Deser, R. Jackiw and S. Templeton, Annals Phys. 140 (1982) 372 [Erratum-ibid. 185 (1982) 406.1988 APNYA,281,409].

[24] A. N. Redlich, Gauge Noninvariance And Parity Violation Of Three-Dimensional Fermions, Phys. Rev. Lett. 52 (1984) 18.

[25] E. Witten, Quantum Field Theory And The Jones Polynomial, Commun. Math. Phys. 121 (1989) 351.

[26] G. W. Moore y N. Seiberg, Taming The Conformal Zoo, Phys. Lett. B 220 (1989) 422.

[27] D. G. Barci, J. F. Medeiros Neto, L. E. Oxman y S. P. Sorella, The point-splitting regularization of $(2+1) d$ parity breaking models, Nucl. Phys. B 600 (2001) 203 [hepth/0011154].

[28] S. D. Deser and A.Ñ. Redlich, $C p^{* * 1}$ - Fermion Correspondence In $D=3$, Phys. Rev. Lett. 61 (1988) 1541. 
[29] S. Deser y R. Jackiw, Higher derivative Chern-Simons extensions, Phys. Lett. B 451 (1999) 73 [hep-th/9901125].

[30] S. R. Coleman y B. Hill, No More Corrections To The Topological Mass Term In QED In Three-Dimensions, Phys. Lett. B 159 (1985) 184.

[31] M. Sakamoto y H. Yamashita, A simple proof of the non-renormalization of the ChernSimons coupling, Phys. Lett. B 476 (2000) 427 [hep-th/9910200].

[32] F. T. Brandt, A. Das y J. Frenkel, Absence of higher order corrections to the non-Abelian Chern-Simons coefficient, Phys. Lett. B494, 339 (2000), hep-th/0009236; Absence of higher order corrections to the non-Abelian topological mass term, Phys. Rev. D63, 085015 (2001) [hep-th/0012087].

[33] S. Elitzur, G. W. Moore, A. Schwimmer and N. Seiberg, Remarks On The Canonical Quantization Of The Chern-Simons-Witten Theory, Nucl. Phys. B 326 (1989) 108.

[34] L. Alvarez-Gaume, J. M. Labastida and A. V. Ramallo, Nucl. Phys. Proc. Suppl. 18B (1991) 1.

[35] A. Achucarro y P. K. Townsend, A Chern-Simons Action For Three-Dimensional AntiDe Sitter Supergravity Theories, Phys. Lett. B 180 (1986) 89.

[36] E. Witten, (2+1)-Dimensional Gravity As An Exactly Soluble System, Nucl. Phys. B 311 (1988) 46.

[37] M. Bañados, C. Teitelboim y J. Zanelli, The Black hole in three-dimensional space-time, Phys. Rev. Lett. 69 (1992) 1849 [hep-th/9204099].

[38] M. Bañados, M. Henneaux, C. Teitelboim y J. Zanelli, Geometry of the (2+1) black hole, Phys. Rev. D 48 (1993) 1506 [gr-qc/9302012].

[39] M. Bañados, Three-dimensional quantum geometry and black holes, , in "Trends in Theoretical Physics II", Buenos Aires (1998) H. Falomir, R. Gamboa Saraví y F.A. Schaposnik eds. AIP, [hep-th/9901148].

[40] O. Coussaert, M. Henneaux y P. van Driel, The Asymptotic dynamics of threedimensional Einstein gravity with a negative cosmological constant, Class. Quant. Grav. 12 (1995) 2961 [gr-qc/9506019].

[41] J. D. Brown y M. Henneaux, Central Charges In The Canonical Realization Of Asymptotic Symmetries: An Example From Three-Dimensional Gravity, Commun. Math. Phys. 104 (1986) 207. 
[42] A. A. Tseytlin, Born-Infeld action, supersymmetry and string theory, [hep-th/9908105]. In Yuri Golfand memorial volume, ed. M. Shifman, World. Sci, 2000.

[43] E.S. Fradkin and A. Tseytlin, Phys. Lett. B163 (1985) 123;

[44] A. A. Tseytlin, On non-abelian generalisation of the Born-Infeld action in string theory, Nucl. Phys. B 501 (1997) 41 [hep-th/9701125].

[45] J. P. Gauntlett, J. Gomis y P. K. Townsend, BPS bounds for worldvolume branes, JHEP 9801 (1998) 003 [hep-th/9711205].

[46] D. Brecher y M. J. Perry, Bound states of D-branes and the non-Abelian Born-Infeld action, Nucl. Phys. B 527 (1998) 121 [hep-th/9801127].

[47] D. Brecher, BPS states of the non-Abelian Born-Infeld action, Phys. Lett. B 442 (1998) 117 [hep-th/9804180].

[48] A. Hashimoto y W. I. Taylor, Fluctuation spectra of tilted and intersecting D-branes from the Born-Infeld action, Nucl. Phys. B 503 (1997) 193 [hep-th/9703217].

[49] C. Callan Jr. and J. Maldacena, Brane dynamics from the Born Infeld action [hep-th/9708147].

[50] J. Polchinski, Tasi Lectures on D-Branes, hep-th/9611050.

[51] W. Taylor IV, Lectures on D-branes, Gauge Theories and M(atrices), [hep-th/9801182].

[52] G.W. Gibbons, Born Infeld particles and Dirichlet p-branes, hep-th/9709027.

[53] S. Gonorazky, F. A. Schaposnik y G. A. Silva, Supersymmetric non-Abelian Born-Infeld theory, Phys. Lett. B 449 (1999) 187 [hep-th/9812094].

[54] G. Landi, An introduction to noncommutative spaces and their geometry, [hep-th/9701078].

[55] D. Bigatti, Non-commutative spaces in physics and mathematics, Class. Quant. Grav. 17 (2000) 3403 [hep-th/0006012].

[56] J. C. Varilly, An introduction to noncommutative geometry, arXiv:physics/9709045.

[57] J. Madore, Noncommutative geometry for pedestrians, [gr-qc/9906059].

[58] L. Castellani, Noncommutative geometry and physics: A review of selected recent results, Class. Quant. Grav. 17 (2000) 3377 [hep-th/0005210].

[59] M. R. Douglas and N. A. Nekrasov, Noncommutative field theory, [hep-th/0106048]. 
[60] R. J. Szabo, Quantum field theory on noncommutative spaces, [hep-th/0109162].

[61] S. Minwalla, M. Van Raamsdonk y N. Seiberg, Noncommutative perturbative dynamics, JHEP 0002 (2000) 020 [hep-th/9912072].

[62] F. Ardalan, H. Arfaei and M. M. Sheikh-Jabbari, Dirac quantization of open strings and noncommutativity in branes, Nucl. Phys. B 576 (2000) 578 [arXiv:hep-th/9906161].

[63] L. Bonora, M. Schnabl, M. M. Sheikh-Jabbari and A. Tomasiello, Noncommutative $S O(n)$ and $S p(n)$ gauge theories, Nucl. Phys. B 589 (2000) 461 [arXiv:hep-th/0006091].

[64] M. Chaichian, P. Presnajder, M. M. Sheikh-Jabbari and A. Tureanu, Noncommutative gauge field theories: A no-go theorem, [ hep-th/0107037].

[65] I. Bars, M. M. Sheikh-Jabbari and M. A. Vasiliev, Noncommutative $O^{*}(N)$ and usp*(2N) algebras and the corresponding gauge field theories, Phys. Rev. D 64 (2001) 086004 [hepth/0103209].

[66] C. Chu, Induced Chern-Simons and WZW action in noncommutative spacetime, Nucl. Phys. B 580 (2000) 352 [hep-th/0003007].

[67] A. A. Bichl, J. M. Grimstrup, V. Putz y M. Schweda, Perturbative Chern-Simons theory on noncommutative $R^{* *} 3$, JHEP0007, 046 (2000), [hep-th/0004071].

[68] N. Grandi y G. A. Silva, Chern-Simons action in noncommutative space, [hepth/0010113].

[69] A. P. Polychronakos, Noncommutative Chern-Simons terms and the noncommutative vacuum, JHEP 0011 (2000) 008 [hep-th/0010264].

[70] G. Chen y Y. Wu, One-loop shift in noncommutative Chern-Simons coupling, Nucl. Phys. B 593 (2001) 562 [hep-th/0006114].

[71] A. Khare y M. B. Paranjape, Solitons in 2+1 dimensional non-commutative Maxwell Chern-Simons Higgs theories, JHEP 0104, 002 (2001), [hep-th/0102016].

[72] G. S. Lozano, E. F. Moreno y F. A. Schaposnik, Self-dual Chern-Simons solitons in noncommutative space, JHEP 0102, 036 (2001), [hep-th/0012266].

[73] D. Bak, S. K. Kim, K. Soh y J. H. Yee, Noncommutative Chern-Simons solitons, [hepth/0102137].

[74] M. M. Sheikh-Jabbari, A note on noncommutative Chern-Simons theories, [hepth/0102092]. 
[75] V. P. Nair y A. P. Polychronakos, On level quantization for the noncommutative ChernSimons theory, [hep-th/0102181].

[76] D. Bak, K. Lee y J. Park, Chern-Simons theories on noncommutative plane, [hepth/0102188].

[77] A. K. Das y M. M. Sheikh-Jabbari, Absence of higher order corrections to noncommutative Chern-Simons coupling, JHEP 0106 (2001) 028 [hep-th/0103139].

[78] A. R. Lugo, A note on the non-commutative Chern-Simons model on manifolds with boundary, [hep-th/0111064].

[79] A. Matusis, L. Susskind y N. Toumbas, The IR/UV connection in the non-commutative gauge theories, JHEP 0012 (2000) 002 [hep-th/0002075].

[80] M. M. Sheikh-Jabbari, Renormalizability of the supersymmetric Yang-Mills theories on the noncommutative torus, JHEP 9906 (1999) 015 [hep-th/9903107].

[81] M. Van Raamsdonk y N. Seiberg, Comments on noncommutative perturbative dynamics, JHEP 0003 (2000) 035 [hep-th/0002186].

[82] K. Furuta y T. Inami, Ultraviolet property of noncommutative Wess-Zumino-Witten model, Mod. Phys. Lett. A 15 (2000) 997 [hep-th/0004024].

[83] E. F. Moreno y F. A. Schaposnik, Wess-Zumino-Witten and fermion models in noncommutative space, Nucl. Phys. B 596 (2001) 439 [hep-th/0008118].

[84] E. F. Moreno y F. A. Schaposnik, The Wess-Zumino-Witten term in non-commutative two-dimensional fermion models, JHEP 0003 (2000) 032 [hep-th/0002236].

[85] J. W. Moffat, Noncommutative quantum gravity, Phys. Lett. B 491 (2000) 345 [hepth/0007181].

[86] J. W. Moffat, Perturbative noncommutative quantum gravity, Phys. Lett. B493, 142 (2000), [hep-th/0008089].

[87] H.Ñishino and S. Rajpoot, Teleparallel complex gravity as foundation for noncommutative gravity, arXiv:hep-th/0107216.

[88] A. H. Chamseddine, Deforming Einstein's gravity, Phys. Lett. B 504 (2001) 33 [hepth/0009153].

[89] A. H. Chamseddine, Complexified gravity in noncommutative spaces, Commun. Math. Phys. 218 (2001) 283 [hep-th/0005222]. 
[90] A. H. Chamseddine, Complex gravity and noncommutative geometry, Int. J. Mod. Phys. A 16 (2001) 759 [hep-th/0010268].

[91] M. Born, On the quantum theory of the electromagnetic field, Proc. Roy. Soc. A143 (1934) 410.

[92] M. Born and L. Infeld, Foundations Of The New Field Theory, Proc. Roy. Soc. Lond. A 144 (1934) 425.

[93] M. Born and L. Infeld, On the quantization of the new field equations I, ibid A147 (1934) 522;

[94] M. Born and L. Infeld, On the quantization of the new field equations II, ibid A150 (1935) 141.

[95] E. Schrödinger, Contribution to Born new theory of the electromagnetic field, Proc. Roy. Soc. A150 (1935) 465.

[96] P.A.M. Dirac, An extensible model of the electron, Proc. Roy. Soc. A268 (1962) 57.

[97] G. Gibbons and D.A. Rasheed,Electric-Magnetic Duality Rotations in Non linear Electrodynamics, Nucl.Phys. B454 (1995) 185.

[98] V. V. Dyadichev and D. V. Gal'tsov, Sphaleron glueballs in NBI theory with symmetrized trace, Nucl. Phys. B 590 (2000) 504 [hep-th/0006242].

[99] S. Deser y R. Puzalowski, Supersymmetric Nonpolynomial Vector Multiplets And Causal Propagation, J. Phys. AA 13 (1980) 2501.

[100] S. Gonorazky, C.Ñunez, F. A. Schaposnik and G. A. Silva, Nucl. Phys. B 531 (1998) 168 [arXiv:hep-th/9805054].

[101] H. R. Christiansen, C. Nunez y F. A. Schaposnik, Uniqueness of Bogomolnyi equations and Born-Infeld like supersymmetric theories, Phys. Lett. B 441 (1998) 185 [hepth/9807197].

[102] Y. Okawa, Derivative corrections to Dirac-Born-Infeld Lagrangian and noncommutative gauge theory, Nucl. Phys. B 566 (2000) 348 [hep-th/9909132].

[103] S. Terashima, On the equivalence between noncommutative and ordinary gauge theories, JHEP 0002 (2000) 029 [hep-th/0001111].

[104] Y. Okawa y S. Terashima, Constraints on effective Lagrangian of D-branes from noncommutative gauge theory, Nucl. Phys. B 584 (2000) 329 [hep-th/0002194]. 
[105] M. R. Garousi, Transformation of the Dirac-Born-Infeld action under the SeibergWitten map, Nucl. Phys. B 602 (2001) 527 [arXiv:hep-th/0011147].

[106] M. R. Garousi, Non-commutative world-volume interactions on D-branes and DiracBorn-Infeld action, Nucl. Phys. B 579 (2000) 209 [hep-th/9909214].

[107] L. Cornalba, Corrections to the Abelian Born-Infeld action arising from noncommutative geometry, JHEP 0009 (2000) 017 [hep-th/9912293].

[108] T. Lee, Noncommutative Dirac-Born-Infeld action for D-brane, Phys. Lett. B 478 (2000) 313 [hep-th/9912038].

[109] J. Pawelczyk, SU(2) WZW D-branes and their noncommutative geometry from DBI action, JHEP 0008 (2000) 006 [hep-th/0003057].

[110] S. Ryang, Open string and Morita equivalence of the Dirac-Born-Infeld action with modulus Phi, [hep-th/0003204].

[111] S. Ferrara y M. A. Lledo, Some aspects of deformations of supersymmetric field theories, JHEP 0005 (2000) 008 [hep-th/0002084].

[112] S. Terashima, A note on superfields and noncommutative geometry, Phys. Lett. B 482 (2000) 276 [hep-th/0002119].

[113] C. Chu y F. Zamora, Manifest supersymmetry in non-commutative geometry, JHEP 0002 (2000) 022 [hep-th/9912153].

[114] S. Cecotti, S. Ferrara y L. Girardello, Flat Potentials In Higher Derivative Supergravity, Phys. Lett. B 187 (1987) 327.

[115] J. D. Lykken, Introduction to supersymmetry, [hep-th/9612114].

[116] N. Nekrasov y A. Schwarz, Instantons on noncommutative $R^{* *} 4$ and $(2,0)$ superconformal six dimensional theory, Commun. Math. Phys. 198 (1998) 689 [hep-th/9802068].

[117] K. Hashimoto, H. Hata y S. Moriyama, Brane configuration from monopole solution in non-commutative super Yang-Mills theory, JHEP 9912 (1999) 021 [hep-th/9910196].

[118] S. Terashima, U(1) instanton in Born-Infeld action and noncommutative gauge theory, Phys. Lett. B 477 (2000) 292 [hep-th/9911245].

[119] H. W. Braden y N. A. Nekrasov, Space-time foam from non-commutative instantons, [hep-th/9912019].

[120] K. Furuuchi, Instantons on noncommutative $R^{* *} 4$ and projection operators, Prog. Theor. Phys. 103 (2000) 1043 [hep-th/9912047]. 
[121] D. Mateos, Non-commutative vs. commutative descriptions of D-brane BIons, Nucl. Phys. B 577 (2000) 139 [hep-th/0002020].

[122] A. Kapustin, A. Kuznetsov y D. Orlov, Noncommutative instantons and twistor transform, Commun. Math. Phys. 221 (2001) 385 [hep-th/0002193].

[123] P. Ho, Twisted bundle on noncommutative space and U(1) instanton, [hep-th/0003012].

[124] K. Kim, B. Lee y H. S. Yang, Comments on instantons on noncommutative $R^{* *} 4$, [hep-th/0003093].

[125] N. Grandi and G. A. Silva, Phys. Lett. B 507 (2001) 345 [arXiv:hep-th/0010113].

[126] M. Banados, O. Chandia, N. Grandi, F. A. Schaposnik and G. A. Silva, Threedimensional noncommutative gravity, Phys. Rev. D 64 (2001) 084012 [arXiv:hepth/0104264].

[127] N. Grandi, R. L. Pakman and F. A. Schaposnik, Nucl. Phys. B 588 (2000) 508 [arXiv:hep-th/0004104]. 